\title{
GENERATING SEQUENCES OF VALUATIONS AND APPLICATIONS
}

\author{
A Dissertation \\ Presented to the Faculty of the Graduate School \\ of University of Missouri-Columbia \\ in Partial Fulfillment of the Requirements for the Degree of \\ Doctor of Philosophy
}

\author{
by \\ PHAM AN VINH \\ Dr. Steven Dale Cutkosky \\ Dissertation Supervisor
}

May 2014 
(C) Copyright by Pham An Vinh 2014 All Rights Reserved 
The undersigned, appointed by the dean of the Graduate School, have examined the thesis entitled

\section{GENERATING SEQUENCES OF VALUATIONS AND APPLICATIONS}

presented by Pham An Vinh, a candidate for the degree of doctor of philosophy, and hereby certify that, in their opinion, it is worthy of acceptance.

Steven Dale Cutkosky

Ian Aberbach

Jerry Nelson

Zhenbo Qin 


\section{ACKNOWLEDGEMENTS}

I am deeply indebted to my advisor Dale Cutkosky and my undergraduate advisor Ngo Viet Trung for providing me with the opportunity to complete my $\mathrm{PhD}$ thesis at the University of Missouri-Columbia. I would like to thank Dale Cutkosky for his kindness, constant support, sympathetic guidance and above all his patience throughout the last six years. Dale and Trung are examples of true mathematicians with expertise, rigor and insights that I always look up to.

I would like to thank all the members of the defense committee, Ian Aberbach, Dale Cutkosky, Jerry Nelson, Zhenbo Qin who carefully examined the draft manuscript.

At the University of Missouri-Columbia, I am very grateful to Ian Aberbach, William Banks, Dale Cutkosky, Zhenbo Qin and Hema Srinivasan for the excellent wide-ranging courses they taught during my graduate years.

My special thanks are due to my parents and my sister for their love, under-

standing and support throughout these long years, during which they had to bear my absence patiently. 


\section{TABLE OF CONTENTS}

Acknowledgements . . . . . . . . . . . . . . . ii

$\begin{array}{ll}\text { Introduction } & 1\end{array}$

1 Preliminaries 4

2 Generating Sequence and Semigroup of a Valuation 6

2.1 Ranks of Valuations under Completion $\ldots \ldots \ldots$

2.2 The Algorithm . . . . . . . . . . . . . . . . . 11

2.3 Valuation Semigroups and Residue Field Extension on a Two Dimensional Regular Local Ring . . . . . . . . . . . . . . . . 32

2.4 Valuation Semigroups on a Regular Local Ring of Dimension Two 40

2.5 Birational Extensions . . . . . . . . . . . . . . . . 43

2.6 Polynomial Rings in two Variables . . . . . . . . . . . . . . 51

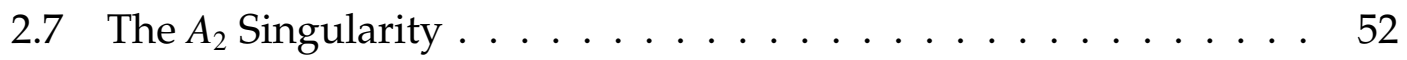

3 Ramification of Local Rings along Valuations $\quad 57$

3.1 Monomial Transforms and Stable Extensions . . . . . . . . . 57

3.2 Stable Extensions along Abhyankar Valuations . . . . . . . . 60

3.3 Stable Extensions along non-Abhyankar Valuations in Dimension

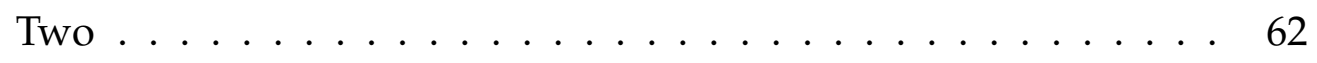

3.4 A modification of the algorithm . . . . . . . . . . 65

3.5 Monomial Forms under Sequences of Quadratic Transforms _. . 68

3.6 Construction of a Generating Sequence in $S$ from that of $R \ldots 73$

4 A sufficient condition for Stable Extensions in Dimension Two 81

4.1 The relation between $t_{0}$ and $t_{1} \ldots \ldots \ldots \ldots$ 
4.2 The sufficient condition for Stable Extensions of Algebraic Local Rings . . . . . . . . . . . . . . . . . . . 83

4.3 Example . . . . . . . . . . . . . . . . . . . . 89

$\begin{array}{lr}\text { Bibliography } & 94\end{array}$

$\begin{array}{ll}\text { Vita } & 98\end{array}$ 


\section{INTRODUCTION}

Valuation Theory arose naturally from Number Theory around the 1910s (see [40]) and soon found important applications in Geometry and Algebra. During the 1940s and the mid 1950s, it was developed intensively and systematically by Zariski, Samuel and Abhyankar (see [51], [2]). The highlights are Zariski's solutions to the problem of resolution of singularities of algebraic surfaces (see [50]) and three-folds in characteristic zero (see [49]) and Abhyankar's solution

for three-folds in characteristic greater than six (see [5]). Cutkosky also simplifies Abhyankar's proof a great deal in [11], while Cossart and Piltant complete the problem for all positive characteristic (see [9] and [10]). In fact, all the known solutions to the problem of resolution of singularities of three-folds in positive characteristic up to this state use valuation theoretic methods: first desingularize the variety near any valuation (this is called local uniformization) and then combine the local uniformizations into a global desingularization. Recently, in [43], Teissier gives a combinatorial proof of embedded local uniformization for rational valuations over equicharacteristic complete Noetherian local rings $R$ whose semigroups on $R$ are finitely generated. This motivates the study of the semigroup of a valuation $v$ on an algebraic local ring $R$ dominated by the valuation ring $V_{v}$, as such semigroup encodes important information about the topology and resolution of singularities of $\operatorname{Spec} R$ and the ideal theory of $R$.

In [42], Spivakovsky gives a characterization of the semigroups of valuations dominating two dimensional algebraic regular local rings with algebraically closed residue fields. He obtains this result by constructing a special generating sequence for such a valuation and applies it to classify sandwiched singularities, which enables desingularization of surfaces over $\mathbb{C}$ by normalized Nash 
transformation (see [41]). The problem of characterizing these semigroups is already a very interesting problem in itself. Spivakovsky's method only works for algebraically closed residue fields and his proof is complex. A simplified version of his construction is provided over $\mathbb{C}$ by Favre and Jonsson in [28]. In [22], we give an algorithm which constructs a special generating sequence for valuations dominating an arbitrary two dimensional regular local ring, and give a complete characterization of the semigroups of such valuations as a corollary. The details will be discussed in Chapter 2 .

Among the important branches of Valuation Theory is the Ramification Theory of Valuations, the generalization of the well-known Ramification Theory of Dedekind Domains. A classic treatment of this theory can be found in [51]. A recent line of development of this theory can be found in Cutkosky's and Cutkosky and Piltant's work (see [13] and [18]). Their central results are the so called Monomialization and Strong Monomialization Theorems. Ghezzi, Ha and Kashcheyeva refine the Strong Monomialization Theorem for algebraic surfaces over algebraically closed ground fields of characteristic zero (see [24]) and Ghezzi and Kashcheyeva extend this result to the case of algebraic surfaces over algebraically closed fields of positive characteristic with defectless extensions of valuations (see [25]). They show that if the extension of algebraic local rings $R \rightarrow S$ along a valuation $v^{*}$ is strongly monomial and stable then the associated graded algebra $\operatorname{gr}_{v^{*}}(S)$ is finitely generated over $\operatorname{gr}_{v}(R)$ (as an algebra). In [21], we generalize the first result to the case of arbitrary ground fields of characteristic zero. We show further that this holds for Abhyankar valuations and ground fields of characteristic zero in any dimension. This will be discussed in Chapter 3. 
In Theorem 6.1 [18], Cutkosky and Piltant prove a deep theorem that the blowing-up process to obtain strong monomialization is eventually stable. This is the key result that is used in Chapter 3 to show that the two generating sequences in a stable extension of algebraic local rings along a valuation $v^{*}$ are essentially the same. In general, it is very hard to indicate when the blowing-up process is actually stable. However, in dimension two, we show that conversely, if the two generating sequences in an extension of algebraic local rings $R \rightarrow S$ along a non-Abhyankar valuation $v^{*}$ is essentially the same then the extension $R_{1} \rightarrow S_{1}$ of the next phase in the full sequences of quadratic transforms of $R$ and $S$ along $v^{*}$ is stable. This gives a sufficient condition for the stability of strongly monomial extensions of algebraic local rings along non-Abyankar valuations in dimension two. This is the content of Chapter 4. 


\section{CHAPTER 1}

\section{PRELIMINARIES}

Suppose that $K$ is a field containing some subfield $₹$ and $\Gamma$ is an ordered abelian group. A map $v: K \rightarrow \Gamma \cup\{\infty\}$ is called a $\mathfrak{f}$-valuation of $K$ if it satisfies the following properties:

1. $v(a b)=v(a)+v(b)$, for all $a, b \in K$;

2. $v(a+b) \geq \min \{v(a), v(b)\}$, for all $a, b \in K$;

3. $v(0)=\infty$ and $\left.v\right|_{\mathfrak{|}\{0\}}=0$.

The local ring $V_{v}=\{a \in K \mid v(a) \geq 0\}$ with its maximal ideal $\mathrm{m}_{v}=\{a \in K \mid$ $v(a)>0\}$ is called the valuation ring of $v$. The quotient $V_{v} / \mathrm{m}_{v}$ is called the residue field of $v$. Since $\left.v\right|_{\mathbb{E}\{0\}}=0, V_{v} / \mathfrak{m}_{v}$ contains $\mathfrak{f}$. The group $\Gamma_{v}=\{v(a) \mid 0 \neq a \in K\}$ is called the value group of $v$.

Next we will introduce the two important invariants of a valuation and their basis properties. For their proofs, we refer to Chapter VI [51] and Chapter II [2]. The rank of $v$ is defined as rank $v=\operatorname{dim} V_{v}$. The rank of $v$ is also the number of strict inclusions of isolated subgroups of $\Gamma_{v}$ and if $v$ has rank $r$ then $\Gamma_{v}$ can be embedded into $\left(\mathbb{R}^{r}\right)_{\text {lex }}$. We say that $v$ is called discrete if $\Gamma_{v} \cong \mathbb{Z}^{r}$.

A set of elements $\gamma_{1}, \ldots, \gamma_{r}$ in $\Gamma_{v}$ is said to be rationally independent if whenever $m_{1} \gamma_{1}+\cdots m_{r} \gamma_{r}=0$ with $m_{i} \in \mathbb{Z}$ then $m_{i}=0$ for all $i$. A rational basis of $\Gamma_{\nu}$ is a maximal rationally independent system of $\Gamma_{\nu}$. Any rational basis of $\Gamma_{\nu}$ has the same cardinality which is defined to be the rational rank rat.rank $v$ of $v$. Another equivalent definition of the rational rank is rat.rank $v=\operatorname{dim}_{\mathbb{Q}} \Gamma_{v} \otimes_{\mathbb{Z}} \mathbb{Q}$ 
We have that rank $v \leq$ rat.rank $v$ and the celebrated Abhyankar's inequality

$$
\text { rat.rank } v+\operatorname{tr} \cdot \operatorname{deg}_{\mathrm{f}} V_{v} / \mathrm{m}_{v} \leq \operatorname{tr} \cdot \operatorname{deg}_{\mathrm{f}} K \text {. }
$$

If equality holds in the last equality, then $\Gamma_{v} \cong \mathbb{Z}^{r}$ as an unordered group, where $r=$ rat.rank $v$. Such valuations are called Abhyankar valuations.

Suppose that $K^{*}$ is an algebraic extension of $K$ and $v^{*}$ is an extension of $v$ to $K^{*}$, i.e. $\left.v^{*}\right|_{K}=v$. Then rank $v=$ rat.rank $v^{*}$ and rat.rank $v=$ rat.rank $v^{*}$. We define, respectively, the reduced ramification index and relative degree of $v^{*}$ over $v$ as

$$
e=\left[\Gamma_{v^{*}}: \Gamma_{v}\right] \text { and } f=\left[V_{v^{*}} / m_{v^{*}}: V_{v} / m_{v}\right]
$$

Moreover, if $K^{*} / K$ is a finite extension then there are finitely many extensions $v^{*}$ of $v$ to $K^{*}$, say $g$ extensions. If char $\mathfrak{f}=0$ and $K^{*} / K$ is Galois then $\left[K^{*}: K\right]=e f g$ and if char $\mathfrak{f}=p$ then $\left[K^{*}: K\right]=e f g p^{\delta\left(v^{*} / v\right)}$, where $\delta\left(v^{*} / v\right)$ is the defect of $v^{*}$ in $K^{*} / K$. If $\left[K^{*}: K\right]=e f g$ then we say that $v^{*} / v$ or $V_{v^{*}} / V_{v}$ is a defectless extension.

Now suppose that $R$ is a subring of $K$ dominated by $v$ (or by $V_{v}$ ), i.e. $R \subset V_{v}$ and $\mathfrak{m}_{v} \cap R=\mathfrak{m}_{R}$. The semigroup $S^{R}(v)=\{v(f) \mid f \in R \backslash\{0\}\}$ is called the semigroup of $v$ in $R$. $S^{R}(v)$ generates $\Gamma_{v}$. For $\gamma \in \Gamma_{v}$, define valuation ideals $\mathcal{P}_{\gamma}(R)=\{f \in R \mid v(f) \geq \gamma\}$ and $\mathcal{P}_{\gamma}^{+}(R)=\{f \in R \mid v(f)>\gamma\}$. We have that $\mathcal{P}_{\gamma}^{+}(R)=\mathcal{P}_{\gamma}(R)$ if and only if $\gamma \notin S^{R}(v)$. The associated graded ring of $v$ on $R$ is

$$
\operatorname{gr}_{v}(R)=\bigoplus_{\gamma \in \Gamma_{v}} \mathcal{P}_{\gamma}(R) / \mathcal{P}_{\gamma}^{+}(R)
$$

Suppose that $f \in R$ and $v(f)=\gamma$. Then the initial form of $f$ in $\operatorname{gr}_{v}(R)$ is $\operatorname{in}_{v}(f)=$ $f+\mathcal{P}_{\gamma}^{+}(R) \in \mathcal{P}_{\gamma}(R) / \mathcal{P}_{\gamma}^{+}(R)$. A set of elements $\left\{f_{i}\right\}_{i \in I}$ such that $\left\{\operatorname{in}_{\gamma}\left(f_{i}\right)\right\}_{i \in I}$ generates $\operatorname{gr}_{v}(R)$ as an $R / \mathfrak{m}_{R}$-algebra is called a generating sequence of $v$ in $R$. 


\section{CHAPTER 2}

\section{GENERATING SEQUENCE AND SEMIGROUP OF A VALUATION}

The main goals of this chapter is to provide an algorithm to construct a special generating sequence of a valuation dominating 2-dimensional algebraic regular local rings and to use such a sequence to characterize the semigroups and the residue fields obtained from such valuations and such rings. The results in this chapter have appeared in [22].

\subsection{Ranks of Valuations under Completion}

In this section we first review the classification of valuations dominating a 2dimensional Noetherian local domain $R$, and extension of the valuation to the completion $\hat{R}$ of $R$. We also discuss the ideal $I_{\hat{R}}$ in $\hat{R}$ of elements of infinitely large value.

Suppose that $\left(R, \mathfrak{m}_{R}\right)$ is a Noetherian local domain of dimension two. Up to order isomorphism, the value groups $\Gamma_{v}$ of a valuation $v$ which dominates $R$ are by Abhyankar's inequality (1.1) and Example 3, Section 15, Chapter VI [51]:

1. $\alpha \mathbb{Z}+\beta \mathbb{Z}$ with $\alpha, \beta \in \mathbb{R}$ rationally independent.

2. $\left(\mathbb{Z}^{2}\right)_{\text {lex }}$.

3. Any subgroup of $\mathbb{Q}$.

Suppose that $N$ is a field, and $V$ is a valuation ring of $N$. We say that the rank of $V$ increases under completion if there exists an analytically normal local 
domain $T$ with quotient field $N$ such that $V$ dominates $T$ and there exists an extension of $V$ to a valuation ring of the quotient field of $\hat{T}$ which dominates $\hat{T}$ and which has higher rank than the rank of $V$.

Theorem 2.1.1 (Theorem 4.2, [17]; [42] in the case when $R / \mathfrak{m}_{R}$ is algebraically closed) Suppose that $V$ dominates an excellent two dimensional local ring $R$. Then the rank of $V$ increases under completion if and only if $V / \mathrm{m}_{V}$ is finite over $R / \mathrm{m}_{R}$ and $V$ is discrete of rank 1 .

Corollary 2.1.2 If $R$ is complete and $v$ is a discrete rank one valuation which dominates $R$ then $\left[V_{v} / \mathfrak{m}_{v}: R / \mathfrak{m}_{R}\right]=\infty$.

The following example shows an important distinction between the case when $R$ is complete and when $R$ is not.

Example 2.1.3 Suppose that $¥$ is a field and $R=\mathfrak{f}[x, y]_{(x, y)}$ is a localization of a polynomial ring in two variables. Then there exists a rank one discrete valuation $v$ dominating $R$ such that $V_{v} / \mathrm{m}_{v}=\mathfrak{f}$.

Proof Let $f(t) \in \mathfrak{E}[[t]]$ be a transcendental element over $\mathfrak{f}(t)$. Embed $R$ into $\mathfrak{f}[[t]]$ by substituting $t$ for $x$ and $f(t)$ for $y$. The valuation $v$ on $R$ obtained by restriction of the $t$-adic valuation to $R$ has the desired properties.

Suppose that $v$ is a valuation which dominates $R$. Let $a$ be the smallest positive element in $S^{R}(v)$. Suppose that $\left\{f_{i}\right\}$ is a Cauchy sequence in $R$ (for the $\mathrm{m}_{R}$-adic topology). Then either

There exist $n_{0} \in \mathbb{Z}_{+}, m \in \mathbb{Z}_{+}$and $\gamma \in S^{R}(v)$ such that $\gamma<m a$ and $v\left(f_{i}\right)=\gamma$ for $i \geq n_{0}$, 
or

Given $m \in \mathbb{Z}_{+}$, there exists $n_{0} \in \mathbb{Z}_{+}$such that $v\left(f_{i}\right)>m a$ for $i>n_{0}$.

Let $I_{\hat{R}}$ be the set of limits of Cauchy sequences $\left\{f_{i}\right\}$ satisfying (2.2). Then $I_{\hat{R}}$ is a prime ideal in $\hat{R}$ ([13], [16], [15], [42], [44]).

Suppose that $\left\{f_{i}\right\}$ is a Cauchy sequence in $R$ which does not satisfy condition (2.2). We will show that condition (2.1) then holds, a fact that we will use in the proof of Proposition 2.1.4 below.

Since the assumption (2.2) does not hold, there exists a positive integer $m$ such that given $n_{0}$, there exists $i_{0}>n_{0}$ such that $v\left(f_{i_{0}}\right) \leq m a$. Since $\left\{f_{i}\right\}$ is a Cauchy sequence for the $\mathrm{m}_{R}$-adic topology in $R$, there exists $n_{0}$ such that $f_{n+1}-f_{n} \in \mathrm{m}_{R}^{m+1}$ for $n>n_{0}$. Thus there exists $i_{0}>n_{0}$ such that $v\left(f_{i_{0}}\right) \leq m a$. For $i>i_{0}$, we have $f_{i}=f_{i_{0}}+h_{i}$ with $h_{i} \in \mathrm{m}_{R}^{m+1} \cdot v\left(f_{i_{0}}\right) \leq m a$ and $v\left(h_{i}\right) \geq(m+1) a$ implies $v\left(f_{i}\right)=v\left(f_{i_{0}}\right)$ for $i \geq i_{0}$. Thus (2.1) holds.

The following proposition is well known.

Proposition 2.1.4 Suppose that $R$ is a regular local ring of dimension two, and let $v$ be a valuation which dominates $R$. Then there exists an extension of $v$ to a valuation $\hat{v}$ which dominates the completion $\hat{R}$ of $R$ with respect to $\mathrm{m}_{R}$, which has one of the following semigroups:

1. $\operatorname{rank} v=\operatorname{rank} \hat{v}=1$ and

$$
S^{R}(v)=S^{\hat{R}}(\hat{v})
$$

2. $v$ is discrete of rank $1, \hat{v}$ is discrete of rank 2 and $S^{\hat{R}}(\hat{v})$ is generated by $S^{R}(v)$ and an element $\alpha$ such that $\alpha>\gamma$ for all $\gamma \in S^{R}(v)$. 
3. $v$ and $\hat{v}$ are discrete of rank 2 , there exists a height one prime $I_{R}$ in $R$, and a discrete rank 1 valuation $\bar{v}$ which dominates the maximal ideal $\mathrm{m}_{R}\left(R / I_{R}\right)$ of $R / I_{R}$ such that

$$
\begin{aligned}
& S^{R}(v) \text { is generated by } S^{R / I_{R}}(\bar{v}) \text { and an element } \alpha \text { such that } \alpha>\gamma \\
& \text { for all } \gamma \in S^{R / I_{R}}(\bar{v}) \text {. } \\
& S^{\hat{R}}(\hat{v}) \text { is generated by } S^{R / I_{R}}(\bar{v}) \text { and an element } \beta \text { such that } \alpha-t \beta \in S^{R / I_{R}}(\bar{v}) \text {, } \\
& \text { for some } t \in \mathbb{Z}_{+} \text {. If } R_{\mathfrak{m}} \text { is excellent, then } t=1 \text {. }
\end{aligned}
$$

4. $v$ and $\hat{v}$ are discrete of rank $2, I_{\hat{R}}=(0)$ and $S^{R}(v)=S^{\hat{R}}(\hat{v})$.

Proof First suppose that $v$ has rank 1 . Then $I_{\hat{R}} \cap R=(0)$, so we have an embedding $R \subset \hat{R} / I_{\hat{R}}$. We can then extend $v$ to a valuation $\bar{v}$ which dominates $\hat{R} / I_{\hat{R}}$ by defining for $f \notin I_{\hat{R}}, \bar{v}\left(f+I_{\hat{R}}\right)=\lim _{i \rightarrow \infty} v\left(f_{i}\right)$, where $\left\{f_{i}\right\}$ is a Cauchy sequence in $R$ representing $f$. Suppose that $0 \neq f+I_{\hat{R}} \in \hat{R} / I_{\hat{R}}$. Then there exists a Cauchy sequence $\left\{f_{i}\right\}$ in $R$ which converges to $f$ and satisfies (2.1) (as shown before the statement of Proposition 2.1.4). Thus $\bar{v}\left(f+I_{\hat{R}}\right) \in S^{R}(v)$, and $S^{R}(v)=S^{\hat{R} / I_{\hat{R}}}(\bar{v})$.

If $I_{\hat{R}}=(0)$ then we have constructed the desired extension $\hat{v}=\bar{v}$ of $v$ to $\hat{R}$. Suppose that $I_{\hat{R}} \neq(0)$. Then $\hat{R} / I_{\hat{R}}$ has dimension 1 , so $\bar{v}$ is discrete of rank 1 . We have that $I_{\hat{R}}=(v)$ is a height one prime ideal. We can extend $\bar{v}$ to a rank 2 valuation $\hat{v}$ which dominates $\hat{R}$ by defining $\hat{v}(f)=(n, \bar{v}(g)) \in\left(\mathbb{Z} \bigoplus \Gamma_{\bar{v}}\right)_{\text {lex }}$ if $f \in \hat{R}$ has a factorization $f=v^{n} g$ where $n \in \mathbb{N}$ and $v \backslash g$.

Now assume that $v$ has rank 2. Further assume that $I_{\hat{R}} \cap R \neq(0)$. Then $v$ has rank 2, and $I_{R}=I_{\hat{R}} \cap R$ is a height one prime ideal in $R$. Thus there exists an irreducible $g \in R$ such that $I_{R}=(g)$. We then have that $I_{\hat{R}}$ is a height one prime ideal in $\hat{R}$, so there exists an irreducible $v \in \hat{R}$ such that $I_{\hat{R}}=(v)$.

There exists a valuation $\bar{v}$ dominating $R / I_{R}$ such that if $f \in R$ has a factoriza- 
tion $f=g^{n} h$ where $g \not h$, then

$$
v(f)=n v(g)+\bar{v}(h)
$$

Write $g=v^{t} \phi$ where $t \in \mathbb{Z}_{+}$and $v \not \phi$. Thus $\phi \notin I_{\hat{R}}$. If $R$ is excellent, then $g$ is reduced in $\hat{R}$ (by Scholie IV 7.8 .3 (vii) [27]), so $t=1$. We have an inclusion $R / I_{R} \subset \hat{R} / I_{\hat{R}}$, and $\bar{v}$ extends to a valuation $\hat{\bar{v}}$ which dominates $\hat{R} / I_{\hat{R}}$. We then extend $v$ to a valuation $\hat{v}$ which dominates $\hat{R}$ by setting

$$
t \hat{v}(v)=v(g)-\hat{\bar{v}}(\phi)
$$

in $\Gamma_{v} \mathbb{R} \cong\left(\mathbb{R}^{2}\right)_{\text {lex }}$. Suppose that $0 \neq f \in \hat{R}$. Factor $f$ as $f=v^{n} h$ where $n \in \mathbb{N}$ and $v \backslash h$. Then define

$$
\hat{v}(f)=n \hat{v}(v)+\hat{\bar{v}}(h)
$$

We now show that $S^{R / I_{R}}(\bar{v})=S^{\hat{R} / I_{\hat{R}}}(\hat{\bar{v}})$. We have that $\hat{\bar{v}}\left(\mathfrak{m}\left(\hat{R} / I_{\hat{R}}\right)\right)=\bar{v}\left(\mathfrak{m}\left(R / I_{R}\right)\right)$. Suppose that $0 \neq h \in \hat{R} / I_{\hat{R}}$, and that $\hat{\bar{v}}(h)=\gamma$. There exists $n \in \mathbb{Z}_{+}$such that $n \hat{\bar{v}}\left(\mathfrak{m}\left(\hat{R} / I_{\hat{R}}\right)\right)>\gamma$ and there exists $f \in R$ such that if $\bar{f}$ is the image of $f$ in $R / I_{R}$, then $\bar{f}-h \in \mathfrak{m}^{n}\left(\hat{R} / I_{\hat{R}}\right)$. Thus $v(f)=\bar{v}(\bar{f})=\hat{\bar{v}}(h)=\gamma$.

Suppose that rank $v=2$ and $I_{\hat{R}} \cap R=(0)$. We can extend $v$ to a valuation $\bar{v}$ dominating $R / I_{\hat{R}}$ by defining for $f \notin I_{\hat{R}}, \bar{v}\left(f+I_{\hat{R}}\right)=\lim _{i \rightarrow \infty} v\left(f_{i}\right)$ if $\left\{f_{i}\right\}$ is a Cauchy sequence in $R$ converging to $f$. We must have that $I_{\hat{R}}=(0)$, since otherwise we would be able to extend $\bar{v}$ to a valuation $\tilde{v}$ dominating $\hat{R}$ which is composite with the rank 2 extension $\bar{v}$ of $v$ to $\hat{R} / I_{\hat{R}}$; this extension would have rank $\geq 3$ which is impossible by Abhyankar's inequality. Thus $I_{\hat{R}}=(0)$.

Remark 2.1.5 Nagata gives an example in (E3.2) of the Appendix to [38] of a regular local ring $R$ of dimension two with an irreducible element $f \in R$ such that $f$ is not reduced in $\hat{R}$. 


\subsection{The Algorithm}

In this section, we will suppose that $R$ is a regular local ring of dimension two, with maximal ideal $\mathfrak{m}_{R}$ and residue field $\mathfrak{f}=R / \mathfrak{m}_{R}$. For $f \in R$, let $\bar{f}$ or $[f]$ denote the residue of $f$ in $f$. Suppose that $v$ is a valuation of the quotient field of $R$ dominating $R$. For $f \in V_{v},[f]$ will denote the class of $f$ in $V_{v} / \mathfrak{m}_{v}$. Since $\mathfrak{f}=$ $R / \mathfrak{m}_{R} \subset V_{v} / \mathfrak{m}_{v}$, the notation $[f]$ for $f \in R$ is consistent.

A coefficient set of $R$ is a subset $W$ of $R$ such that the mapping $W \rightarrow \mathfrak{f}$ defined by $s \mapsto \bar{s}$ is a bijection. We further require that $0 \in W$ and $1 \in W$. Suppose that $C S$ is a coefficient set of $R$.

Remark 2.2.1 Suppose that $x, y$ are regular parameters in $R, a, b \in C S$ and $n \in \mathbb{Z}_{+}$. Let $c \in C S$ be defined by $\overline{a+b}=\bar{c}$. Then there exist $e_{i j} \in C S$ such that

$$
a+b=c+\sum_{i+j=1}^{n-1} e_{i j} x^{i} y^{j}+h
$$

with $h \in \mathfrak{m}_{R^{n}}^{n}$ Let $d \in C S$ be defined by $\overline{a b}=\bar{d}$. Then there exist $g_{i j} \in C S$ such that

$$
a b=d+\sum_{i+j=1}^{n-1} g_{i j} x^{i} y^{j}+h^{\prime}
$$

with $h^{\prime} \in \mathfrak{m}_{R}^{n}$.

Lemma 2.2.2 Suppose that $\Gamma$ is a totally ordered abelian group, I is a finite or countable index set of cardinality $\geq 2$ and $\beta_{i} \in \Gamma$ are positive elements for $i \in I$. Let $\Lambda=|I|-1$. Let

$$
\bar{n}_{i}=\left[G\left(\beta_{0}, \ldots, \beta_{i}\right): G\left(\beta_{0}, \ldots, \beta_{i-1}\right)\right] \in \mathbb{Z}_{+} \cup\{\infty\}
$$

for $\geq 1$. Assume that $\bar{n}_{i} \in \mathbb{Z}_{+}$if $i<\Lambda$. Let $s_{i}$ be the smallest positive integer $t$ such that $t \beta_{i} \in S\left(\beta_{0}, \ldots, \beta_{i-1}\right)$ (or $s_{i}=\infty$ if $i=\Lambda$ and no such $t$ exists). 
Suppose that $1 \leq k<\Lambda$ and $\bar{n}_{i} \beta_{i}<\beta_{i+1}$ for $1 \leq i \leq k-1$. Then

1) $s_{i}=\bar{n}_{i}$ for $1 \leq i \leq k$.

2) If $\gamma \in G\left(\beta_{0}, \ldots, \beta_{k}\right)$ and $\gamma \geq \bar{n}_{k} \beta_{k}$ then $\gamma \in S\left(\beta_{0}, \ldots, \beta_{k}\right)$.

Proof We first prove 2). By repeated Euclidean division, we obtain an expansion $\gamma=a_{0} \beta_{0}+a_{1} \beta_{1}+\cdots+a_{k} \beta_{k}$ with $a_{0} \in \mathbb{Z}$ and $0 \leq a_{i}<\bar{n}_{i}$ for $1 \leq i \leq k$. Now we calculate, using the inequalities $\bar{n}_{i} \beta_{i}<\beta_{i+1}$,

$$
a_{1} \beta_{1}+\cdots+a_{k} \beta_{k}<\bar{n}_{k} \beta_{k} .
$$

Thus $a_{0}>0$ and $\gamma \in S\left(\beta_{0}, \ldots, \beta_{k}\right)$.

Now 1) follows from 2) and induction on $k$.

Theorem 2.2.3 Suppose that $v$ is a valuation of the quotient field of $R$ dominating $R$. Let $L=V_{v} / \mathfrak{m}_{v}$ be the residue field of the valuation ring $V_{v}$ of $v$. Suppose that $x, y$ are regular parameters in $R$. Then there exist $\Omega \in \mathbb{Z}_{+} \cup\{\infty\}$ and $P_{i} \in \mathfrak{m}_{R}$ for $i \in \mathbb{Z}_{+}$with $i<\min \{\Omega+1, \infty\}$ such that $P_{0}=x, P_{1}=y$ and for $1 \leq i<\Omega$, there is an expression

$$
P_{i+1}=P_{i}^{n_{i}}+\sum_{k=1}^{\lambda_{i}} c_{k} P_{0}^{\sigma_{i, 0}(k)} P_{1}^{\sigma_{i, 1}(k)} \cdots P_{i}^{\sigma_{i, i}(k)}
$$

with $n_{i} \geq 1, \lambda_{i} \geq 1$,

$$
0 \neq c_{k} \in C S
$$

for $1 \leq k \leq \lambda_{i}, \sigma_{i, s}(k) \in \mathbb{N}$ for all $s, k, 0 \leq \sigma_{i, s}(k)<n_{s}$ for $s \geq 1$. Further,

$$
n_{i} v\left(P_{i}\right)=v\left(P_{0}^{\sigma_{i, 0}(k)} P_{1}^{\sigma_{i, 1}(k)} \cdots P_{i}^{\sigma_{i, i}(k)}\right)
$$

for all $k$.

For all $i \in \mathbb{Z}_{+}$with $i<\Omega$, the following are true: 
1) $v\left(P_{i+1}\right)>n_{i} v\left(P_{i}\right)$.

2) Suppose that $r \in \mathbb{N}$ is less than $\Omega, m \in \mathbb{Z}_{+}, j_{k}(l) \in \mathbb{N}$ for $1 \leq l \leq m$ and $0 \leq j_{k}(l)<n_{k}$ for $1 \leq k \leq r$ are such that $\left(j_{0}(l), j_{1}(l), \ldots, j_{r}(l)\right)$ are distinct for $1 \leq l \leq m$, and

$$
v\left(P_{0}^{j_{0}(l)} P_{1}^{j_{1}(l)} \cdots P_{r}^{j_{r}(l)}\right)=v\left(P_{0}^{j_{0}(1)} \cdots P_{r}^{j_{r}(1)}\right)
$$

for $1 \leq l \leq m$. Then

$$
1,\left[\frac{P_{0}^{j_{0}(2)} P_{1}^{j_{1}(2)} \cdots P_{r}^{j_{r}(2)}}{P_{0}^{j_{0}(1)} P_{1}^{j_{1}(1)} \cdots P_{r}^{j_{r}(1)}}\right], \cdots,\left[\frac{P_{0}^{j_{0}(m)} P_{1}^{j_{1}(m)} \cdots P_{r}^{j_{r}(m)}}{P_{0}^{j_{0}(1)} P_{1}^{j_{1}(1)} \cdots P_{r}^{j_{r}(1)}}\right]
$$

are linearly independent over $\mathfrak{\mathfrak { f }}$.

3) Let

$$
\bar{n}_{i}=\left[G\left(v\left(P_{0}\right), \ldots, v\left(P_{i}\right)\right): G\left(v\left(P_{0}\right), \ldots, v\left(P_{i-1}\right)\right)\right] .
$$

Then $\bar{n}_{i}$ divides $\sigma_{i, i}(k)$ for all $k$ in (2.6). In particular, $n_{i}=\bar{n}_{i} d_{i}$ with $d_{i} \in \mathbb{Z}_{+}$.

4) There exists $U_{i}=P_{0}^{w_{0}(i)} P_{1}^{w_{1}(i)} \ldots P_{i-1}^{w_{i-1}(i)}$ for $i \geq 1$ with $w_{0}(i), \ldots, w_{i-1}(i) \in \mathbb{N}$ and $0 \leq w_{j}(i)<n_{j}$ for $1 \leq j \leq i-1$ such that $v\left(P_{i}^{\bar{n}_{i}}\right)=v\left(U_{i}\right)$ and if

$$
\alpha_{i}=\left[\frac{P_{i}^{\bar{n}_{i}}}{U_{i}}\right]
$$

then

$$
b_{i, t}=\left[\sum_{\sigma_{i, i}(k)=t \bar{n}_{i}} c_{k} \frac{P_{0}^{\sigma_{i, 0}(k)} P_{1}^{\sigma_{i, 1}(k)} \cdots P_{i-1}^{\sigma_{i, i-1}(k)}}{U_{i}^{\left(d_{i}-t\right)}}\right] \in \mathfrak{F}\left(\alpha_{1}, \ldots, \alpha_{i-1}\right)
$$

for $0 \leq t \leq d_{i}-1$ and

$$
f_{i}(u)=u^{d_{i}}+b_{i, d_{i}-1} u^{d_{i}-1}+\cdots+b_{i, 0}
$$

is the minimal polynomial of $\alpha_{i}$ over $\mathfrak{f}\left(\alpha_{1}, \ldots, \alpha_{i-1}\right)$.

The algorithm terminates with $\Omega<\infty$ if and only if either

$$
\bar{n}_{\Omega}=\left[G\left(v\left(P_{0}\right), \ldots, v\left(P_{\Omega}\right)\right): G\left(v\left(P_{0}\right), \ldots, v\left(P_{\Omega-1}\right)\right)\right]=\infty
$$


or

$$
\begin{aligned}
& \left.\bar{n}_{\Omega}<\infty\left(\text { so that } \alpha_{\Omega} \text { is defined as in } 4\right)\right) \text { and } \\
& d_{\Omega}=\left[\mathfrak{f}\left(\alpha_{1}, \ldots, \alpha_{\Omega}\right): \mathfrak{f}\left(\alpha_{1}, \ldots, \alpha_{\Omega-1}\right)\right]=\infty .
\end{aligned}
$$

If $\bar{n}_{\Omega}=\infty$, set $\alpha_{\Omega}=1$.

Proof Consider the following statements $A(i), B(i), C(i), D(i)$ for $1 \leq i<\Omega$ :

There exists $U_{i}=P_{0}^{w_{0}(i)} P_{1}^{w_{1}(i)} \cdots P_{i-1}^{w_{i-1}(i)}$ for some $w_{j}(i) \in \mathbb{N}$ and $0 \leq w_{j}(i)<n_{j}$ for $1 \leq j \leq i-1$ such that $\bar{n}_{i} v\left(P_{i}\right)=v\left(U_{i}\right)$. Let $\alpha_{i}=\left[\frac{P_{i}^{\bar{n}_{i}}}{U_{i}}\right] \in L$ and

$A(i) \quad f_{i}(u)=u^{d_{i}}+b_{i, d_{i}-1} u^{d_{i}-1}+\cdots+b_{i, 0} \in \mathfrak{f}\left(\alpha_{1}, \ldots, \alpha_{i-1}\right)[u]$ be the minimal polynomial of $\alpha_{i}$.

Let $d_{i}$ be the degree of $f_{i}(u)$. Then there exist $a_{s, t} \in C S$ and $j_{0}(s, t), j_{1}(s, t), \ldots, j_{i-1}(s, t) \in \mathbb{N}$ with $0 \leq j_{k}(s, t)<n_{k}$ for $k \geq 1$ and $0 \leq t<d_{i}$ such that $v\left(P_{0}^{j_{0}(s, t)} P_{1}^{j_{1}(s, t)} \cdots P_{i-1}^{j_{i-1}(s, t)} P_{i}^{t \bar{n}_{i}}\right)=\bar{n}_{i} d_{i} v\left(P_{i}\right)$ for all $s, t$ and

$$
P_{i+1}:=P_{i}^{\bar{n}_{i} d_{i}}+\sum_{t=0}^{d_{i}-1}\left(\sum_{s=1}^{\lambda_{t}} a_{s, t} P_{0}^{j_{0}(s, t)} P_{1}^{j_{1}(s, t)} \cdots P_{i-1}^{j_{i-1}(s, t)}\right) P_{i}^{t \bar{n}_{i}}
$$

satisfies

$$
b_{i, t}=\left[\sum_{s=1}^{\lambda_{t}} a_{s, t} \frac{P_{0}^{j_{0}(s, t)} P_{1}^{j_{1}(s, t)} \cdots P_{i-1}^{j_{i-1}(s, t)}}{U_{i}^{d_{i}-t}}\right]
$$

for $0 \leq t \leq d_{i}-1$. In particular, $n_{i}=\bar{n}_{i} d_{i}$ and

$$
v\left(P_{i+1}\right)>n_{i} v\left(P_{i}\right)
$$


$B(i)$ Suppose that $M$ is a Laurent monomial in $P_{0}, P_{1}, \ldots, P_{i}$ and $v(M)=0$. Then there exist $s_{i} \in \mathbb{Z}$ such that

$$
M=\prod_{j=1}^{i}\left[\frac{P_{j}^{\bar{j}_{j}}}{U_{j}}\right]^{s_{j}},
$$

so that

$$
[M] \in \mathfrak{f}\left(\alpha_{1}, \ldots, \alpha_{i}\right) .
$$

Suppose that $\lambda \in \mathfrak{f}\left(\alpha_{1}, \ldots, \alpha_{i}\right)$ and $N$ is a Laurent monomial in $P_{0}, P_{1}, \ldots, P_{i}$ such that $\gamma=v(N) \geq n_{i} v\left(P_{i}\right)$. Then there exists

$$
G=\sum_{j} c_{j} P_{0}^{\tau_{0}(j)} P_{1}^{\tau_{1}(j)} \cdots P_{i}^{\tau_{i}(j)}
$$

with $\tau_{0}(j), \ldots, \tau_{i}(j) \in \mathbb{N}, 0 \leq \tau_{k}(j)<n_{k}$ for $1 \leq k \leq i$ and $c_{j} \in C S$ such that

$$
v\left(P_{0}^{\tau_{0}(j)} P_{1}^{\tau_{1}(j)} \cdots P_{i}^{\tau_{i}(j)}\right)=\gamma \text { for all } j
$$

and

$$
\left[\frac{G}{N}\right]=\lambda .
$$

Suppose that $m \in \mathbb{Z}_{+}, j_{k}(l) \in \mathbb{N}$ for $1 \leq l \leq m$ and $0 \leq j_{k}(l)<n_{k}$

for $1 \leq k \leq i$ are such that the $\left(j_{0}(l), j_{1}(l), \ldots, j_{i}(l)\right)$ are distinct for $1 \leq l \leq m$, and

$$
v\left(P_{0}^{j_{0}(l)} P_{1}^{j_{1}(l)} \cdots P_{i}^{j_{i}(l)}\right)=v\left(P_{0}^{j_{0}(1)} \cdots P_{i}^{j_{i}(1)}\right)
$$

$D(i)$ for $1 \leq l \leq m$. Then

$$
1,\left[\frac{P_{0}^{j_{0}(2)} P_{1}^{j_{1}(2) \ldots P_{i}^{j_{i}(2)}}}{P_{0}^{j_{0}(1)} P_{1}^{j_{1}(1)} \ldots P_{i}^{j_{i}(1)}}\right], \ldots,\left[\frac{P_{0}^{j_{0}(m)} P_{1}^{j_{1}(m)} \ldots P_{i}^{j_{i}(m)}}{P_{0}^{j_{0}(1)} P_{1}^{j_{1}(1)} \ldots P_{i}^{j_{i}(1)}}\right]
$$

are linearly independent over $\mathrm{f}$.

We will leave the proofs of $A(1), B(1), C(1)$ and $D(1)$ to the reader, as they are an easier variation of the following inductive statement, which we will prove. 
Assume that $i \geq 1$ and $A(i), B(i), C(i)$ and $D(i)$ are true. We will prove that $A(i+1), B(i+1)$ and $C(i+1)$ and $D(i+1)$ are true. Let $\beta_{j}=v\left(P_{j}\right)$ for $0 \leq j \leq i+1$. By Lemma 2.2.2, there exists $U_{i+1}=P_{0}^{w_{0}(i)} P_{1}^{w_{1}(i)} \cdots P_{i}^{w_{i}(i)}$ for some $w_{j}(i) \in \mathbb{N}$ such that $0 \leq w_{j}(i)<n_{j}$ for $1 \leq j \leq i$ and $v\left(U_{i+1}\right)=\bar{n}_{i+1} \beta_{i+1}$ (where $\bar{n}_{i+1}=\left[G\left(\beta_{0}, \ldots, \beta_{i+1}\right)\right.$ : $\left.\left.G\left(\beta_{0}, \ldots, \beta_{i}\right)\right]\right)$.

Let $f_{i+1}(u)$ be the minimal polynomial of

$$
\alpha_{i+1}=\left[\frac{P_{i+1}^{\bar{n}_{i+1}}}{U_{i+1}}\right]
$$

over $\mathfrak{f}\left(\alpha_{1}, \ldots, \alpha_{i}\right)$. Let $d=d_{i+1}=\operatorname{deg} f_{i+1}$. Expand

$$
f_{i+1}(u)=u^{d}+b_{d-1} u^{d-1}+\cdots+b_{0}
$$

with $b_{j} \in \mathfrak{F}\left(\alpha_{1}, \ldots, \alpha_{i}\right)$. For $j \geq 1$,

$$
v\left(U_{i+1}^{j}\right)=j \bar{n}_{i+1} \beta_{i+1} \geq \beta_{i+1}>n_{i} \beta_{i} .
$$

In the inductive statement $C(i)$, take $N=U_{i+1}^{d-t}$ for $0 \leq t<d=d_{i+1}$, to obtain for $0 \leq t<d_{i+1}$

$$
G_{t}=\sum_{s=1}^{\lambda_{t}} a_{s, t} P_{0}^{j_{0}(s, t)} P_{1}^{j_{1}(s, t)} \cdots P_{i}^{j_{i}(s, t)}
$$

with $a_{s, t} \in C S, j_{k}(s, t) \in \mathbb{N}$ and $0 \leq j_{k}(s, t)<n_{k}$ for $1 \leq k \leq i$ such that

$$
v\left(G_{t}\right)=v\left(P_{0}^{j_{0}(s, t)} P_{1}^{j_{1}(s, t)} \cdots P_{i}^{j_{i}(s, t)}\right)=(d-t) \bar{n}_{i+1} \beta_{i+1}
$$

for all $s, t$ and

$$
\left[\frac{G_{t}}{U_{i+1}^{d-t}}\right]=b_{t}
$$

Set

$$
\begin{aligned}
P_{i+2} & =P_{i+1}^{\bar{n}_{i+1} d_{i+1}}+G_{d-1} P_{i+1}^{\bar{n}_{i+1}\left(d_{i+1}-1\right)}+\cdots+G_{0} \\
& =P_{i+1}^{\bar{n}_{i+1} d_{i+1}}+\sum_{t=0}^{d-1} \sum_{s=1}^{\lambda_{t}} a_{s, t} P_{0}^{j_{0}(s, t)} P_{1}^{j_{1}(s, t)} \cdots P_{i}^{j_{i}(s, t)} P_{i+1}^{\bar{n}_{i+1}} .
\end{aligned}
$$

We have established $A(i+1)$. 
Suppose $M$ is a Laurent polynomial in $P_{0}, P_{1}, \ldots, P_{i+1}$ and $v(M)=0$. We have a factorization

$$
M=P_{0}^{a_{0}} P_{1}^{a_{1}} \cdots P_{i}^{a_{i}} P_{i+1}^{a_{i+1}}
$$

with all $a_{j} \in \mathbb{Z}$. Thus $a_{i+1} \beta_{i+1} \in G\left(\beta_{0}, \ldots, \beta_{i}\right)$, so that $\bar{n}_{i+1}$ divides $a_{i+1}$. Let $s=\frac{a_{i+1}}{\bar{n}_{i+1}}$. Then

$$
M=U_{i+1}^{s}\left(P_{0}^{a_{0}} P_{1}^{a_{1}} \cdots P_{i}^{a_{i}}\right)\left(\frac{P_{i+1}^{\bar{n}_{i+1}}}{U_{i+1}}\right)^{s} .
$$

Now $U_{i+1}^{s} P_{0}^{a_{0}} \cdots P_{i}^{a_{i}}$ is a Laurent monomial in $P_{0}, \ldots, P_{i}$ of value zero, so the validity of $B(i+1)$ follows from the inductive assumption $B(i)$.

We now establish $C(i+1)$. Suppose $\lambda \in \mathfrak{f}\left(\alpha_{1}, \ldots, \alpha_{i+1}\right)$ and $N$ is a Laurent monomial in $P_{0}, P_{1}, \ldots, P_{i+1}$ such that $\gamma=v(N) \geq n_{i+1} v\left(P_{i+1}\right)$. We have

$$
\gamma \geq n_{i+1} \beta_{i+1}=\bar{n}_{i+1} d_{i+1} \beta_{i+1} \geq \bar{n}_{i+1} \beta_{i+1} .
$$

By Lemma 2.2.2 there exist $r_{0}, r_{1}, \ldots, r_{i}, k \in \mathbb{N}$ such that $0 \leq r_{j}<n_{j}$ for $1 \leq j \leq i$ and $0 \leq k<\bar{n}_{i+1}$ such that

$$
\bar{N}=P_{0}^{r_{0}} P_{1}^{r_{1}} \cdots P_{i}^{r_{i}} P_{i+1}^{k}
$$

satisfies $v(\bar{N})=\gamma$. Let $\tilde{N}=P_{0}^{r_{0}} P_{1}^{r_{1}} \cdots P_{i}^{r_{i}}$, so that $\bar{N}=\tilde{N} P_{i+1}^{k}$. Let $\tau=\left[\frac{N}{\bar{N}}\right]$. We have that $0 \neq \tau \in \mathfrak{f}\left(\alpha_{1}, \ldots, \alpha_{i+1}\right)$ by $B(i+1)$.

Suppose $0 \leq j \leq d_{i+1}-1$. Then

$$
\begin{aligned}
v\left(\frac{\tilde{N}}{U_{i+1}^{j}}\right) & =v(\tilde{N})-j v\left(U_{i+1}\right) \\
& \geq \gamma-\left(\bar{n}_{i+1}-1\right) \beta_{i+1}-\left(d_{i+1}-1\right) \bar{n}_{i+1} \beta_{i+1} \\
& \geq \bar{n}_{i+1} d_{i+1} \beta_{i+1}-\bar{n}_{i+1} \beta_{i+1}+\beta_{i+1}-d_{i+1} \bar{n}_{i+1} \beta_{i+1}+\bar{n}_{i+1} \beta_{i+1} \\
& \geq \beta_{i+1}>n_{i} \beta_{i} .
\end{aligned}
$$

Write

$$
\tau \lambda=e_{0}+e_{1} \alpha_{i+1}+\cdots+e_{d_{i+1}-1} \alpha_{i+1}^{d_{i+1}-1}
$$


with $e_{j} \in \mathfrak{f}\left(\alpha_{1}, \ldots, \alpha_{i}\right)$. By the inductive statement $C(i)$ and (2.15), there exist for $0 \leq j \leq d_{i+1}-1$

$$
H_{j}=\sum_{k} c_{k, j} P_{0}^{\delta_{0}(k, j)} P_{1}^{\delta_{1}(k, j)} \cdots P_{i}^{\delta_{i}(k, j)}
$$

with $\delta_{0}(k, j), \delta_{1}(k, j), \ldots, \delta_{i}(k, j) \in \mathbb{N}, 0 \leq \delta_{l}(k, j)<n_{l}$ for $1 \leq l$ and $c_{k, j} \in C S$ for all $k, j$ such that

$$
v\left(P_{0}^{\delta_{0}(k, j)} P_{1}^{\delta_{1}(k, j)} \cdots P_{i}^{\delta_{i}(k, j)}\right)=v\left(\frac{\tilde{N}}{U_{i+1}^{j}}\right)
$$

for all $j, k$ and

$$
\left[\frac{H_{j}}{\left(\frac{\tilde{N}}{U_{i+1}^{j}}\right)}\right]=e_{j}
$$

for all $j$. Set

$$
G=H_{0} P_{i+1}^{k}+H_{1} P_{i+1}^{\bar{n}_{i+1}+k}+\cdots+H_{d_{i+1}-1} P_{i+1}^{\bar{n}_{i+1}\left(d_{i+1}-1\right)+k} .
$$

We have

$$
\bar{n}_{i+1}\left(d_{i+1}-1\right)+k<\bar{n}_{i+1}\left(d_{i+1}-1\right)+\bar{n}_{i+1} \leq \bar{n}_{i+1} d_{i+1}=n_{i+1},
$$

and

$$
\frac{G}{\bar{N}}=\frac{H_{0}}{\tilde{N}}+\left(\frac{H_{1} U_{i+1}}{\tilde{N}}\right)\left(\frac{P_{i+1}^{\bar{n}_{i+1}}}{U_{i+1}}\right)+\cdots+\left(\frac{H_{d_{i+1}-1} U_{i+1}^{d_{i+1}-1}}{\tilde{N}}\right)\left(\frac{P_{i+1}^{\bar{n}_{i+1}}}{U_{i+1}}\right)^{d_{i+1}-1} .
$$

We have

$$
\left[\frac{G}{\bar{N}}\right]=e_{0}+e_{1} \alpha_{i+1}+\cdots+e_{d_{i+1}-1} \alpha_{i+1}^{d_{i+1}-1}=\tau \lambda .
$$

Thus

$$
\left[\frac{G}{N}\right]=\left[\begin{array}{l}
G \\
\bar{N}
\end{array}\right]\left[\begin{array}{l}
\bar{N} \\
\bar{N}
\end{array}\right]=\tau \lambda \tau^{-1}=\lambda .
$$

We have established $C(i+1)$.

Suppose that $D(i+1)$ is not true. We will obtain a contradiction. Under the assumption that $D(i+1)$ is not true, there exists $m \in \mathbb{Z}_{+}, j_{k}(l) \in \mathbb{N}$ for $1 \leq l \leq m$ with $0 \leq j_{k}(l)<n_{k}$ for $1 \leq k \leq i+1$ such that $\left(j_{0}(l), j_{1}(l), \ldots, j_{i+1}(l)\right)$ are distinct for 
$1 \leq l \leq m$, and

$$
v\left(P_{0}^{j_{0}(l)} P_{1}^{j_{1}(l)} \cdots P_{i+1}^{j_{i+1}(l)}\right)=v\left(P_{0}^{j_{0}(1)} P_{1}^{j_{0}(1)} \cdots P_{i+1}^{j_{i+1}(1)}\right)
$$

for $1 \leq l \leq m$ and $\tilde{a}_{l} \in \mathfrak{f}$ for $1 \leq l \leq m$ not all zero such that

$$
\tilde{a}_{1}+\tilde{a}_{2}\left[\frac{P_{0}^{j_{0}(2)} P_{1}^{j_{1}(2)} \cdots P_{i+1}^{j_{i+1}(2)}}{P_{0}^{j_{0}(1)} P_{1}^{j_{1}(1)} \cdots P_{i+1}^{j_{i+1}(1)}}\right]+\cdots+\tilde{a}_{m}\left[\frac{P_{0}^{j_{0}(m)} P_{1}^{j_{1}(m)} \cdots P_{i+1}^{j_{i+1}(m)}}{P_{0}^{j_{0}(1)} P_{1}^{j_{1}(1)} \cdots P_{i+1}^{j_{i+1}(1)}}\right]=0 .
$$

We may assume that all $\tilde{a}_{l} \neq 0$. We have that $\left(j_{i+1}(l)-j_{i+1}(1)\right) \beta_{i+1} \in G\left(\beta_{0}, \ldots, \beta_{i}\right)$ for $1 \leq l \leq m$, so $\bar{n}_{i+1}$ divides $\left(j_{i+1}(l)-j_{i+1}(1)\right)$ for all $l$. Thus after possibly dividing all monomials $P_{0}^{j_{0}(l)} P_{1}^{j_{1}(l)} \cdots P_{i+1}^{j_{i+1}(l)}$ by a common power of $P_{i+1}$, we may assume that

$$
\bar{n}_{i+1} \text { divides } j_{i+1}(l) \text { for all } l \text {. }
$$

After possibly reindexing the $P_{0}^{j_{0}(l)} P_{1}^{j_{1}(l)} \cdots P_{i+1}^{j_{i+1}(l)}$, we may assume that $j_{i+1}(1)=$ $\bar{n}_{i+1} \phi$ is the largest value of $j_{i+1}(l)$.

For $1 \leq l \leq m$, define $a_{l} \in C S$ by $\bar{a}_{l}=\tilde{a}_{l}$. Let

$$
Q=\sum_{l=1}^{m} a_{l} P_{0}^{j_{0}(l)} P_{1}^{j_{1}(l)} \cdots P_{i+1}^{j_{i+1}(l)}
$$

Let

$$
Q_{s}=\sum_{j_{i+1}(l)=s \bar{n}_{i+1}} a_{l} P_{0}^{j_{0}(l)} P_{1}^{j_{1}(l)} \cdots P_{i}^{j_{i}(l)}
$$

for $0 \leq s \leq \phi$. Then

$$
Q=\sum_{s=0}^{\phi} Q_{s} P_{i+1}^{\bar{n}_{i+1} s}
$$

Let

$$
c_{s}=\left[\frac{Q_{s}}{P_{0}^{j_{0}(1)} P_{1}^{j_{1}(1)} \ldots P_{i}^{j_{i}(1)} U_{i+1}^{(\phi-s)}}\right] \in \mathfrak{f}\left(\alpha_{1}, \ldots \alpha_{i}\right)
$$

by $B(i)$. Now

$$
c_{\phi}=\sum_{j_{i+1}(l)=\phi \bar{n}_{i+1}} \tilde{a}_{l}\left[\frac{P_{0}^{j_{0}(l)} \cdots P_{i}^{j_{i}(l)}}{P_{0}^{j_{0}(1)} \cdots P_{i}^{j_{i}(1)}}\right]
$$

and the monomials $P_{0}^{j_{0}(l)} \cdots P_{i}^{j_{i}(l)}$ with $j_{i+1}(l)=\phi \bar{n}_{i+1}\left(\right.$ which includes $P_{0}^{j_{0}(1)} \cdots P_{i}^{j_{i}(1)}$ ) all have the same value and their exponents satisfy the condition $0 \leq j_{k}(l)<$ 
$n_{k}$ for $0<k$. Since these monomials are necessarily distinct and the $\tilde{a}_{l}$ are all nonzero, by $D(i)$ we have that $c_{\phi} \neq 0$.

Dividing $Q$ by $P_{0}^{j_{0}(1)} P_{1}^{j_{1}(1)} \cdots P_{i}^{j_{i}(1)} U_{i+1}^{\phi}$, we compute

$$
\begin{aligned}
& \sum_{s=1}^{\phi} c_{s} \alpha_{i+1}^{s}=\sum_{s=1}^{\phi} c_{s}\left[\frac{P_{i+1}^{\bar{n}_{i+1}}}{U_{i+1}}\right]^{s} \\
& =\sum_{s=1}^{\phi}\left[\frac{Q_{s}}{P_{0}^{j_{0}(1)} \cdots P_{i}^{j_{i}(1)} U_{i+1}^{(\phi-s)}}\right]\left[\frac{P_{i+1}^{\bar{n}_{i+1}}}{U_{i+1}}\right]^{S} \\
& =\sum_{s=1}^{\phi}\left[\frac{Q_{s} P_{i+1}^{\bar{n}_{i+1}}}{P_{0}^{j_{0}(1)} \ldots P_{i}^{j_{i}(1)} U_{i+1}^{\phi}}\right] \\
& =\sum_{s=1}^{\phi} \sum_{j_{i+1}(l)=s \bar{n}_{i+1}} \tilde{a}_{l}\left[\frac{P_{0}^{j_{0}(l)} \ldots P_{i}^{j_{i}(l)} P_{i+1}^{\bar{n}_{i+1}}}{P_{0}^{j_{0}(1)} \ldots P_{i}^{j_{i}(1)} P_{i+1}^{j_{i+1}(1)}}\right]\left[\frac{P_{i+1}^{j_{i+1}^{(1)}}}{U_{i+1}^{\phi}}\right] \\
& =\left(\sum_{l=1}^{m} \tilde{a}_{l}\left[\frac{P_{0}^{j_{0}(l) \ldots P_{i}^{j_{i}(l)}} P_{i+1}^{j_{i+1}(l)}}{P_{0}^{j_{0}(1)} \ldots P_{i}^{j_{i}(1)} P_{i+1}^{j_{i+1}(1)}}\right]\right) \alpha_{i+1}^{\phi}=0 .
\end{aligned}
$$

Note that

$$
\left[\frac{P_{i+1}^{j_{i+1}(1)}}{U_{i+1}^{\phi}}\right]=\left[\frac{P_{i+1}^{\bar{n}_{i+1}}}{U_{i+1}}\right]^{\phi}=\alpha_{i+1}^{\phi} .
$$

Thus the minimal polynomial $f_{i+1}(u)$ of $\alpha_{i+1}$ divides $g(u)=\sum_{s=0}^{\phi} c_{s} u^{s}$ in $\mathfrak{f}\left(\alpha_{1}, \ldots, \alpha_{i}\right)[u]$. But then $\phi \geq d_{i+1}$, so that $j_{i+1}(1)=\bar{n}_{i+1} \phi \geq n_{i+1}$, a contradiction.

Remark 2.2.4 Theorem 2.2.3 can be stated without recourse to a coefficient set. To give this statement (which has the same proof) (2.7) must be replaced with " $c_{k}$ are units in $R$ for $1 \leq k \leq \lambda_{i}$ ". In the proof, the statement " $a_{s, t} \in C S$ " in $A(i)$ must be replaced with " $a_{s, t}$ units in $R$ or $a_{s, t}=0$ ". The statement " $c_{j} \in C S$ " in $C(i)$ must be replaced with " $c_{j}$ is a unit in $R$ or $c_{j}=0$ ".

Remark 2.2.5 For $i>0$, there is an expression

$$
P_{i+1}=y^{n_{1} \cdots n_{i}}+x \Theta_{i+1}
$$

with $\Theta_{i+1} \in R$. This follows by considering the expression (2.6) and the various constraints on the values of the terms of the monomials in this expression. 
Remark 2.2.6 The algorithm of Theorem 2.2.3 concludes with $\Omega<\infty$ if and only if $v\left(P_{\Omega}\right) \notin \mathbb{Q} v(x)$ (so that rank $(v)=2$ ) or $v$ is discrete of rank 1 with $\operatorname{trdeg} g_{R / \mathrm{m}_{R}} V_{v} / \mathrm{m}_{v}=1$ (so that $v$ is divisorial).

Proof From Theorem 2.2.3, we see that the algorithm terminates with $\Omega<\infty$ if and only if either

$$
\left[G\left(v\left(P_{0}\right), \ldots, v\left(P_{\Omega}\right)\right): G\left(v\left(P_{0}\right), \ldots, v\left(P_{\Omega-1}\right)\right)\right]=\infty
$$

or

$\left[G\left(v\left(P_{0}\right), \ldots, v\left(P_{\Omega}\right)\right): G\left(v\left(P_{0}\right), \ldots, v\left(P_{\Omega-1}\right)\right)\right]<\infty$ and $\left[\mathfrak{f}\left(\alpha_{1}, \ldots, \alpha_{\Omega}\right): \mathfrak{f}\left(\alpha_{1}, \ldots, \alpha_{\Omega-1}\right)\right]=\infty$

Remark 2.2.7 Suppose that $\Omega=\infty$ and $n_{i}=1$ for $i \gg 0$ in the conclusions of Theorem 2.2.3. Then $v$ is discrete, and $V_{v} / \mathfrak{m}_{v}$ is finite over $\mathrm{f}$.

Proof We first deduce a consequence of the assumption that $\Omega=\infty$ and $n_{i}=1$ for $i \gg 0$. There exists $i_{0} \in \mathbb{Z}_{+}$such that $n_{i}=1$ for all $i \geq i_{0}$. Thus for $i \geq i_{0}, P_{i+1}$ is the sum of $P_{i}$ and a f-linear combination of monomials $M$ in $x$ and the finitely many $P_{j}$ with $j<i_{0}$, and with $v(M)=v\left(P_{i}\right)$. We see that the $P_{i}$ form a Cauchy sequence in $\hat{R}$ whose limit $f$ in $\hat{R}$ is nonzero (by Remark 2.2.5), and such that $\lim _{i \rightarrow \infty} v\left(P_{i}\right)=\infty$.

Thus $I_{\hat{R}} \neq(0), v$ is discrete and $V_{v} / \mathrm{m}_{v}$ is finite over $\mathrm{f}$ by the proof of Proposition 2.1.4.

Remark 2.2.8 Suppose that $V_{v} / \mathrm{m}_{v}=R / \mathrm{m}_{R}$ in the hypotheses of Theorem 2.2.3 (so that there is no residue field extension). Then the $P_{i}$ constructed by the algorithm are 
binomials for $i \geq 2$; (2.6) becomes

$$
P_{i+1}=P_{i}^{\bar{n}_{i}}+c U_{i}=P_{i}^{\bar{n}_{i}}+c P_{0}^{w_{0}(i)} \cdots P_{i-1}^{w_{i-1}(i)}
$$

for some $0 \neq c \in C S$.

Example 2.2.9 There exists a rank 2 valuation $v$ dominating $R=\mathfrak{f}[x, y]_{(x, y)}$ such that the set

$$
\left\{v\left(P_{0}\right), v\left(P_{1}\right), v\left(P_{2}\right), \ldots\right\}
$$

does not generate the semigroup $S^{R}(v)$.

Proof Suppose that $\mathfrak{f}$ is a field of characteristic zero. We define a rank 2 valuation $\hat{v}$ on $\mathfrak{f}[[x, y]]$. Let $g(x, y)=y-x \sqrt{x+1}$. For $0 \neq f(x, y) \in \mathfrak{f}[[x, y]]$, we have a factorization $f=g^{n} h$ where $n \in \mathbb{N}$ and $g \backslash h$. The rule

$$
\hat{v}(f)=(n, \operatorname{ord}(h(x, x \sqrt{1+x}))) \in\left(\mathbb{Z}^{2}\right)_{\operatorname{lex}}
$$

then defines a rank 2 valuation dominating $\mathfrak{f}[[x, y]]$ with value group $\left(\mathbb{Z}^{2}\right)_{\text {lex }}$.

We have that $(g) \cap \mathfrak{F}[x, y]=\left(y^{2}-x^{2}-x^{3}\right)$. Thus $\hat{v}$ restricts to a rank 2 valuation $v$ which dominates the maximal ideal $\mathfrak{n}=(x, y)$ of $\mathfrak{f}[x, y]$. Expand

$$
x \sqrt{1+x}=\sum_{j \geq 1} a_{i} x^{j}=x+\frac{1}{2} x^{2}-\frac{1}{8} x^{3}+\cdots
$$

as a series with all $a_{j} \in \mathfrak{f}$ non zero. Applying the algorithm of Theorem 2.2.3, we construct the infinite sequence of polynomials $P_{1}, P_{2}, \cdots$ where $P_{0}=x, P_{1}=y$ and $P_{i}=y-\sum_{j=1}^{i-1} a_{i} x^{i}$ for $i \geq 2$. We have that $v\left(P_{i}\right)=(0, i)$ for $i \geq 0$. However, $v\left(y^{2}-x^{2}-x^{3}\right)=(1,1)$.

Thus the set $\left\{v(x), v\left(P_{1}\right), v\left(P_{2}\right), \ldots\right\}$ does not generate the semigroup $S^{R}(v)$. 
Lemma 2.2.10 Suppose that $v$ is a valuation dominating $R$. Let

$$
P_{0}=x, P_{1}=y, P_{2}, \ldots
$$

be the sequence of elements of $R$ constructed by Theorem 2.2.3. Set $\beta_{i}=v\left(P_{i}\right)$ for $i \geq 0$. Suppose that $P_{0}^{m_{0}} P_{1}^{m_{1}} \cdots P_{r}^{m_{r}}$ is a monomial in $P_{0}, \ldots, P_{r}$ and $m_{i} \geq n_{i}$ for some $i \geq 1$. Let $\rho=v\left(P_{0}^{m_{0}} P_{1}^{m_{1}} \cdots P_{r}^{m_{r}}\right)$. Then with the notation of (2.10),

$$
\begin{aligned}
P_{0}^{m_{0}} \cdots P_{r}^{m_{r}}= & -\sum_{t=0}^{d_{i}-1} \sum_{s=1}^{\lambda_{t}} a_{s, t} P_{0}^{m_{0}+j_{0}(s, t)} \cdots P_{i-1}^{m_{i-1}+j_{i-1}(s, t)} P_{i}^{m_{i}-n_{i}+t \bar{n}_{i}} P_{i+1}^{m_{i+1}} \cdots P_{r}^{m_{r}} \\
& +P_{0}^{m_{0}} \cdots P_{i}^{m_{i}-n_{i}} P_{i+1}^{m_{i+1}+1} \cdots P_{r}^{m_{r}}
\end{aligned}
$$

All terms in the first sum of (2.19) have value $\rho$ and $v\left(P_{0}^{m_{0}} \cdots P_{i}^{m_{i}-n_{i}} P_{i+1}^{m_{i+1}+1} \cdots P_{r}^{m_{r}}\right)>\rho$.

Suppose that $W$ is a Laurent monomial in $P_{0}, \ldots, P_{r}$ such that $v(W)=\rho$. Then

$$
\left[\frac{P_{0}^{m_{0}} P_{1}^{m_{1}} \cdots P_{r}^{m_{r}}}{W}\right]=-\sum_{t=0}^{d_{i}-1} \sum_{s=1}^{\lambda_{t}} \bar{a}_{s, t}\left[\frac{P_{0}^{m_{0}+j_{0}(s, t)} \cdots P_{i-1}^{m_{i-1}+j_{i-1}(s, t)} P_{i}^{m_{i}-n_{i}+\bar{n}_{i} t} P_{i+1}^{m_{i+1}} \cdots P_{r}^{m_{r}}}{W}\right]
$$

and

$$
\begin{aligned}
& \left(m_{0}+j_{0}(s, t)\right)+\cdots+\left(m_{i-1}+j_{i-1}(s, t)\right)+\left(m_{i}-n_{i}+t \bar{n}_{i}\right)+m_{i+1}+\cdots+m_{r} \\
& >m_{0}+m_{1}+\cdots+m_{r}
\end{aligned}
$$

for all terms in the first sum of (2.19).

Proof We have

$$
P_{0}^{m_{0}} \cdots P_{r}^{m_{r}}=P_{0}^{m_{0}} \cdots P_{i}^{n_{i}} P_{i}^{m_{i}-n_{i}} \cdots P_{r}^{m_{r}}
$$

where $m_{i}-n_{i} \geq 0$. Substituting (2.10) for $P_{i}^{n_{i}}$, we obtain equation (2.19). We 
compute, from the first term of (2.19),

$$
\begin{aligned}
& -\sum_{t=1}^{d_{i}-1} \sum_{s=1}^{\lambda_{t}} \bar{a}_{s, t}\left[\frac{P_{0}^{m_{0}+j_{0}(s, t)} \cdots P_{r}^{m_{r}}}{W}\right] \\
& =-\left[\frac{P_{0}^{m_{0}} \ldots P_{i}^{m_{i}-n_{i}} \ldots P_{r}^{m_{r}} U_{i}^{d_{i}}}{W}\right]\left(\sum_{t=0}^{d_{i}-1} \sum_{s=1}^{\lambda_{t}} \bar{a}_{s, t}\left[\frac{P_{0}^{j_{0}(s, t)} \cdots P_{i-1}^{j_{i-1}(s, t)}}{U_{i}^{d_{i}-t}}\right]\left[\frac{P_{i}^{\bar{n}_{i}}}{U_{i}}\right]^{t}\right)
\end{aligned}
$$

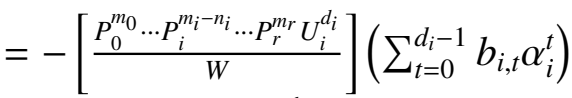

$$
\begin{aligned}
& =\left[\frac{P_{0}^{m_{0}} \ldots P_{i}^{m_{i}-n_{i}} \ldots P_{r}^{m_{r}} U_{i}^{d_{i}}}{W}\right] \alpha_{i}^{d_{i}} \\
& =\left[\frac{P_{0}^{m_{0}} \ldots P_{i}^{m_{i}-n_{i} \ldots P_{r}^{m_{r}}} U_{i}^{d_{i}}}{W}\right]\left[\frac{P_{i}^{\bar{n}_{i}}}{U_{i}}\right]^{d_{i}} \\
& =\left[\frac{P_{0}^{m_{0}} \cdots P_{i}^{m_{i}} \cdots P_{r}^{m_{r}}}{W}\right] \text {, }
\end{aligned}
$$

giving (2.20). For all $s, t$ (with $0 \leq t \leq d_{i}-1$ ),

$$
\begin{aligned}
n_{i} \beta_{i} & =j_{0}(s, t) \beta_{0}+j_{1}(s, t) \beta_{1}+\cdots+j_{i-1}(s, t) \beta_{i-1}+\bar{n}_{i} t \beta_{i} \\
& <\left(j_{0}(s, t)+j_{1}(s, t)+\cdots+j_{i-1}(s, t)+\bar{n}_{i} t\right) \beta_{i}
\end{aligned}
$$

so

$$
n_{i}<j_{0}(s, t)+j_{1}(s, t)+\cdots+j_{i-1}(s, t)+\bar{n}_{i} t
$$

(2.21) follows.

Theorem 2.2.11 Suppose that $v$ is a valuation dominating $R$. Let

$$
P_{0}=x, P_{1}=y, P_{2}, \ldots
$$

be the sequence of elements of $R$ constructed by Theorem 2.2.3. Set $\beta_{i}=v\left(P_{i}\right)$ for $i \geq 0$. Suppose that $f \in R$ and there exists $n \in \mathbb{Z}_{+}$such that $v(f)<n v\left(\mathrm{~m}_{R}\right)$. Then there exists an expansion

$$
f=\sum_{I} a_{I} P_{0}^{i_{0}} P_{1}^{i_{1}} \cdots P_{r}^{i_{r}}+\sum_{J} \phi_{J} P_{0}^{j_{0}} \cdots P_{r}^{j_{r}}+h
$$

where $r \in \mathbb{N}, a_{I} \in C S, I=\left(i_{0}, i_{1}, \ldots, i_{r}\right), J=\left(j_{0}, j_{1}, \ldots, j_{r}\right) \in \mathbb{N}^{r+1}, v\left(P_{0}^{i_{0}} P_{1}^{i_{1}} \ldots P_{r}^{i_{r}}\right)=$ $v(f)$ for all $I$ in the first sum, $0 \leq i_{k}<n_{k}$ for $1 \leq k \leq r, v\left(P_{0}^{j_{0}} \cdots P_{r}^{j_{r}}\right)>v(f)$ for all terms in the second sum, $\phi_{J} \in R$ and $h \in \mathrm{m}_{R}^{n}$. 
The first sum is uniquely determined by these conditions.

Proof We first prove existence. We have an expansion

$$
f=\sum a_{i_{0}, i_{1}} x^{i_{0}} y^{i_{1}}+h_{0}
$$

with $a_{i_{0}, i_{1}} \in C S$ and $h_{0} \in \mathfrak{m}_{R}^{n}$. More generally, suppose that we have an expansion

$$
f=\sum a_{I} P_{0}^{i_{0}} P_{1}^{i_{1}} \cdots P_{r}^{i_{r}}+h
$$

for some $r \in \mathbb{Z}_{+}, I=\left(i_{0}, \ldots, i_{r}\right) \in \mathbb{N}^{r+1}, a_{I} \in C S$ and $h \in \mathfrak{m}_{R^{n}}^{n}$. Let

$$
\rho=\min \left\{v\left(P_{0}^{i_{0}} P_{1}^{i_{1}} \cdots P_{r}^{i_{r}}\right) \mid a_{I} \neq 0\right\} .
$$

We can rewrite (2.22) as

$$
f=\sum_{J} a_{J} P_{0}^{j_{0}} P_{1}^{j_{1}} \cdots P_{r}^{j_{r}}+\sum_{J^{\prime}} a_{J^{\prime}} P_{0}^{j_{0}^{\prime}} P_{1}^{j_{1}^{\prime}} \cdots P_{r}^{j_{r}^{\prime}}+h
$$

where the terms in the first sum have minimal value $v\left(P_{0}^{j_{0}} P_{1}^{j_{1}} \cdots P_{r}^{j_{r}}\right)=\rho$ and the nonzero terms in the second sum have value $v\left(P_{0}^{j_{0}^{\prime}} P_{1}^{j_{1}^{\prime}} \cdots P_{r}^{j_{r}^{\prime}}\right)>\rho$.

If we have that the first sum is nonzero and $0 \leq j_{k}<n_{k}$ for $1 \leq k \leq r$ for all terms in the first sum of (2.23) then $\rho=v(f)$ and we have achieved the conclusions of the theorem. So suppose that one of these conditions fails.

First suppose that $\sum_{J} a_{J} P_{0}^{j_{0}} \cdots P_{r}^{j_{r}} \neq 0$ and for some $J, j_{i} \geq n_{i}$ for some $i \geq 1$. Let

$$
a=\min \left\{j_{0}+\cdots+j_{r} \mid j_{i} \geq n_{i} \text { for some } i \geq 1\right\}
$$

and let $b$ be the numbers of terms in $\sum_{J} a_{J} P_{0}^{j_{0}} \cdots P_{r}^{j_{r}}$ such that $j_{i} \geq n_{i}$ for some $i \geq 1$ and $j_{0}+\cdots+j_{r}=a$. Let $\sigma=(a, b) \in\left(\mathbb{Z}^{2}\right)_{\text {lex }}$. Let $J_{0}=\left(\bar{j}_{0}, \ldots, \bar{j}_{r}\right)$ be such that $a_{J_{0}} \neq 0$ and $\bar{j}_{0}+\cdots+\bar{j}_{r}=a$. Write

$$
P_{0}^{\overline{j_{0}}} \cdots P_{r}^{\bar{j}_{r}}=P_{0}^{\bar{j}_{0}} \cdots P_{i}^{\bar{j}_{i}-n_{i}} P_{i}^{n_{i}} \cdots P_{r}^{\bar{j}_{r}}
$$


and substitute (2.10) for $P_{i}^{n_{i}}$, to obtain an expression of the form (2.19) of Lemma 2.2.10. Substitute this expression (2.19) for $P_{0}^{\bar{j}_{0}} \cdots P_{r}^{\bar{j}_{r}}$ in (2.23) and apply Remark 2.2.1, to obtain an expression of the form (2.23) such that either the first sum is zero or the first sum is nonzero and all terms in the first sum satisfy $j_{i}<n_{i}$ for $1 \leq i$ so that $v(f)=\rho$ and we have achieved the conclusions of the theorem, or the first sum has a nonzero term which satisfies $j_{i} \geq n_{i}$ for some $i \geq 1$. By (2.21), we have an increase in $\sigma$ if this last case holds.

Since there are only finitely many monomials $M$ in $P_{0}, \ldots P_{r}$ which have the value $\rho$, after a finite number of iterations of this step we must either find an expression (2.23) where the first sum is zero, or attain an expression (2.23) satisfying the conclusions of the theorem.

If we obtain an expression (2.23) where the first sum is zero, then we have an expression (2.22) with an increase in $\rho$ (and possibly an increase in $r$ ), and we repeat the last step, either attaining the conclusions of the theorem or obtaining another increase in $\rho$. Since there are only a finite number of monomials in the $\left\{P_{i}\right\}$ which have value $\leq v(f)$, we must achieve the conclusions of the theorem in a finite number of steps.

Uniqueness of the first sum follows from 2) of Theorem 2.2.3.

Theorem 2.2.12 Suppose that $v$ is a rank 1 valuation which dominates $R$ and $v(x)=$ $v\left(\mathfrak{m}_{R}\right)$. Then

a) The set $\left\{\operatorname{in}_{v}(x)\right\} \cup\left\{\operatorname{in}_{v}\left(P_{i}\right) \mid n_{i}>1\right\}$ minimally generates $\operatorname{gr}_{v}(R)$ as a $\mathrm{f}$-algebra.

b) The set

$$
\{v(x)\} \cup\left\{v\left(P_{i}\right) \mid \bar{n}_{i}>1\right\}
$$


minimally generates the semigroup $S^{R}(v)$.

c) $V_{v} / \mathfrak{m}_{v}=\mathfrak{f}\left(\alpha_{i} \mid d_{i}>1\right)$ where $\alpha_{i}$ is defined by 4) (and possibly (2.9)) of Theorem 2.2.3.

Proof Theorem 2.2.11 implies that the set $\left\{\operatorname{in}_{v}(x)\right\} \cup\left\{\operatorname{in}_{v}\left(P_{i}\right) \mid n_{i}>1\right\}$ generates $\operatorname{gr}_{v}(R)$ as a $\mathrm{f}$-algebra. We will show that the set generates $\operatorname{gr}_{v}(R)$ minimally. Suppose that it doesn't. Then there exists an $i \in \mathbb{N}$ such that $n_{i}>1$ if $i>0$ and a sum

$$
H=\sum_{J} c_{J} P_{0}^{j_{0}} \cdots P_{r}^{j_{r}}
$$

for some $r \in \mathbb{N}$ with $c_{J} \in C S$ such that the monomials $P_{0}^{j_{0}} \cdots P_{r}^{j_{r}}$ have value $v\left(P_{0}^{j_{0}} \cdots P_{r}^{j_{r}}\right)=v\left(P_{i}\right)$ with $j_{i}=0$ and $j_{k}=0$ if $n_{k}=1$ for $1 \leq k \leq r$ for all $J$, and

$$
v\left(\sum_{J} c_{J} P_{0}^{j_{0}} \cdots P_{r}^{j_{r}}-P_{i}\right)>v\left(P_{i}\right) .
$$

We thus have by 1 ) of Theorem 2.2.3 and since $v\left(P_{0}\right)=v\left(\mathfrak{m}_{R}\right)$, that $r \leq i-1$. Thus $i \geq 1$. By Theorem 2.2.11 applied to $H$, we have an expression

$$
P_{i}=\sum_{K} d_{K} P_{0}^{k_{0}} \cdots P_{s}^{k_{s}}+f
$$

where $s \in \mathbb{N}, d_{K} \in C S, 0 \leq k_{l}<n_{l}$ for $1 \leq l$, some $d_{K} \neq 0, f \in R$ is such that $v(f)>v\left(P_{i}\right)$, and

$$
v\left(P_{0}^{k_{0}} \cdots P_{s}^{k_{s}}\right)=v(H)=v\left(P_{i}\right)
$$

for all monomials in the first sum of (2.25). Since the minimal value terms of the expression of $H$ in (2.24) only involve $P_{0}, \ldots, P_{i-1}$ and all these monomials have the same value $\rho=v(H)$, the algorithm of Theorem 2.2.11 ends with $s \leq i-1$ in (2.25). But then we obtain from (2.25) a contradiction to 2) of Theorem 2.2.3.

Now a) and 3) of Theorem 2.2.3 imply statement b). 
Suppose that $0 \neq \lambda \in L=V_{v} / \mathrm{m}_{v}$. Then $\lambda=\left[\frac{f}{f^{\prime}}\right]$ for some $f, f^{\prime} \in R$ since $R$ and $V_{v}$ have the same quotient field. We have that $v(f)=v\left(f^{\prime}\right)$. By Theorem 2.2.11, there exist $r \in \mathbb{Z}_{+}$and expressions

$$
\begin{aligned}
& f=\sum_{i=1}^{m} a_{i} P_{0}^{\sigma_{0}(i)} P_{1}^{\sigma_{1}(i)} \cdots P_{r}^{\sigma_{r}(i)}+h, \\
& f^{\prime}=\sum_{j=1}^{n} b_{j} P_{0}^{\tau_{0}(j)} P_{1}^{\tau_{1}(j)} \cdots P_{r}^{\tau_{r}(j)}+h^{\prime}
\end{aligned}
$$

with $a_{i}, b_{j} \in C S, 0 \leq \sigma_{k}(i)<n_{k}$ for $1 \leq k$ and $0 \leq \tau_{k}(j)<n_{k}$ for $1 \leq k$, the $P_{0}^{\sigma_{0}(i)} P_{1}^{\sigma_{1}(i)} \cdots P_{r}^{\sigma_{r}(i)}, P_{0}^{\tau_{0}(j)} P_{1}^{\tau_{1}(j)} \cdots P_{r}^{\tau_{r}(j)}$ all have the common value

$$
\rho:=v(f)=v\left(f^{\prime}\right)
$$

$h, h^{\prime} \in R$ and $v(h)>\rho, v\left(h^{\prime}\right)>\rho$.

$$
\begin{aligned}
\lambda=\left(\sum_{i} \bar{a}_{i}\left[P_{0}^{\sigma_{0}(i)-\sigma_{0}(1)} \cdots P_{r}^{\sigma_{r}(i)-\sigma_{r}(1)}\right]\right)\left(\sum_{j} \bar{b}_{j}\left[P_{0}^{\tau_{0}(i)-\sigma_{0}(1)} \cdots P_{r}^{\tau_{r}(i)-\sigma_{r}(1)}\right]\right)^{-1} \\
\quad \in \mathfrak{F}\left(\alpha_{1}, \ldots, \alpha_{r}\right)
\end{aligned}
$$

by $B(r)$ of the proof of Theorem 2.2.3.

If $V_{v} / \mathfrak{m}_{v}$ is transcendental over $\mathfrak{f}$ then $\Gamma_{v} \cong \mathbb{Z}$ by Abhyankar's inequality. Zariski called such a valuation a "prime divisor of the second kind". By c) of Theorem 2.2.12, $V_{v} / \mathfrak{m}_{v}=\mathfrak{f}\left(\alpha_{i} \mid d_{i}>1\right)$. There thus exists an index $i$ such that $\mathfrak{f}\left(\alpha_{1}, \ldots, \alpha_{i-1}\right)$ is algebraic over $\mathfrak{f}$ and $\alpha_{i}$ is transcendental over $\mathfrak{f}\left(\alpha_{1}, \ldots, \alpha_{i-1}\right)$. Thus $\Omega=i$ in the algorithm of Theorem 2.2.3, since $\alpha_{i}$ does not have a minimal polynomial over $\mathfrak{f}\left(\alpha_{1}, \ldots, \alpha_{i-1}\right)$.

Theorem 2.2.13 Suppose that $v$ is a rank 2 valuation which dominates $R$ and $v(x)=$ $v\left(\mathfrak{m}_{R}\right)$. Let $I_{v}$ be the height one prime ideal in $V_{v}$. Then one of the following three cases hold: 
1. $I_{v} \cap R=\mathfrak{m}_{R}$. Then

a) the finite set

$$
\left\{\operatorname{in}_{v}(x)\right\} \cup\left\{\operatorname{in}_{v}\left(P_{i}\right) \mid n_{i}>1\right\}
$$

minimally generates $\operatorname{gr}_{v}(R)$ as an $\mathrm{f}$-algebra and

b) the finite set

$$
\{v(x)\} \cup\left\{v\left(P_{i}\right) \mid \bar{n}_{i}>1\right\}
$$

minimally generates the semigroup $S^{R}(v)$.

c) $V_{v} / \mathfrak{m}_{v}=\mathfrak{f}\left(\alpha_{i} \mid d_{i}>1\right)$.

2. $I_{v} \cap R=\left(P_{\Omega}\right)$ is a height one prime ideal in $R$ and

a) the finite set

$$
\left\{\operatorname{in}_{v}(x)\right\} \cup\left\{\operatorname{in}_{v}\left(P_{i}\right) \mid n_{i}>1\right\}
$$

minimally generates $\operatorname{gr}_{v}(R)$ as a $\mathfrak{k}$-algebra, and

b) The finite set

$$
\{v(x)\} \cup\left\{v\left(P_{i}\right) \mid \bar{n}_{i}>1\right\}
$$

minimally generates the semigroup $S^{R}(v)$.

c) $V_{v} / \mathfrak{m}_{v}=\mathfrak{f}\left(\alpha_{i} \mid d_{i}>1\right)$.

3. $I_{v} \cap R=(g)$ is a height one prime ideal in $R$ and

a) the finite set

$$
\left\{\operatorname{in}_{v}(x)\right\} \cup\left\{\operatorname{in}_{v}\left(P_{i}\right) \mid n_{i}>1\right\} \cup\left\{\operatorname{in}_{v}(g)\right\}
$$

minimally generates $\operatorname{gr}_{v}(R)$ as a $\mathfrak{f}$-algebra, and

b) The finite set

$$
\{v(x)\} \cup\left\{v\left(P_{i}\right) \mid \bar{n}_{i}>1\right\} \cup\{v(g)\}
$$

minimally generates the semigroup $S^{R}(v)$. 
c) $V_{v} / \mathfrak{m}_{v}=\mathfrak{f}\left(\alpha_{i} \mid d_{i}>1\right)$.

Proof Since $v$ has $\operatorname{rank} 2$, the set $\left\{P_{i} \mid n_{i}>1\right\}$ is a finite set since otherwise either $\Gamma_{v}$ is not a finitely generated group or $V_{v} / \mathrm{m}_{v}$ is not a finitely generated field extension of $\mathfrak{1}$, by 3 ) and 4 ) of Theorem 2.2.3, which is a contradiction to Abhyankar's inequality.

The case when $I_{v} \cap R=\mathrm{m}_{R}$ now follows from Theorem 2.2.11 and 2), 3) of Theorem 2.2.3; the proof of $\mathrm{c}$ ) is the same as the proof of $\mathrm{c}$ ) of Theorem 2.2.12.

Suppose that $I_{v} \cap R=(g)$ is a height one prime ideal in $R$. Suppose that $h \in R$. Then there exists $n \in \mathbb{N}$ and $u \in R$ such that $h=g^{n} u$ with $u \notin(g)$. Thus

$$
v(h)=n v(g)+v(u)
$$

Assume that $\Omega<\infty$. Then $v\left(P_{\Omega}\right) \notin \mathbb{Q} v\left(\mathrm{~m}_{R}\right)$ by Remark 2.2.6. Thus $P_{\Omega} \in I_{v} \cap R$, so $P_{\Omega}=g f$ for some $f \in R$. We will show that $f$ is a unit in $R$. Suppose not. Then $v(g)<v\left(P_{\Omega}\right)$. Let $t=\operatorname{ord}(g)$. There exists $c \in \mathbb{Z}_{+}$such that if $j_{0}, j_{1}, \ldots, j_{\Omega-1} \in \mathbb{N}$ are such that $v\left(P_{0}^{j_{0}} P_{1}^{j_{1}} \cdots P_{\Omega-1}^{j_{\Omega-1}}\right) \geq c v\left(m_{R}\right)$ then $\operatorname{ord}\left(P_{0}^{j_{0}} P_{1}^{j_{1}} \cdots P_{\Omega-1}^{j_{\Omega-1}}\right)>t$. We may assume that $c$ is larger than $t$. Write

$$
g=\sum_{i, j=1}^{c} a_{i j} x^{i} y^{j}+\Lambda
$$

with $\Lambda \in \mathfrak{m}_{R}^{c}$ and $a_{i j} \in C S . g$ has an expression of the form

$$
g=\sum_{J} a_{J} P_{0}^{j_{0}} \cdots P_{\Omega}^{j_{\Omega}}+\sum_{J^{\prime}} a_{J^{\prime}} P_{0}^{j_{0}^{\prime}} \cdots P_{\Omega}^{j_{\Omega^{\prime}}}+h
$$

with $a_{J}, a_{J^{\prime}} \in C S$ and $h \in \mathfrak{m}_{R^{\prime}}^{c}$, and the terms in the first sum all have a common value $\rho$, which is smaller than the values of the terms in the second sum. 
Now we draw some conclusions which must hold for an expression of the form (2.27). We must have that

$$
\rho<c v\left(\mathfrak{m}_{R}\right)
$$

since otherwise, by our choice of $c$ and our assumption that $\operatorname{ord}(f)>0$, so that $\operatorname{ord}\left(P_{\Omega}\right)>\operatorname{ord}(g)=t$, we would have that the right hand side of (2.27) has order larger than $t$, which is impossible. In particular, we have

$$
j_{\Omega}=0
$$

in all terms in the first sum.

We also must have that

$$
j_{i} \geq n_{i} \text { for some } i \text { with } 1 \leq i<\Omega \text { for all terms in the first sum. }
$$

This follows since otherwise we would have $v(g)=\rho<c v\left(\mathfrak{m}_{R}\right)$, which is impossible.

We apply the algorithm of Theorem 4.9 to (2.27), and apply a substitution of the form (20) to a monomial in the first sum. As shown in the proof of Theorem 4.9 , we must obtain an expression (2.27) with an increase in $\rho$ after a finite number of iterations, since (2.30) must continue to hold. Since there are only finitely many values in the semigroup $S^{R}(v)$ between 0 and $c v\left(\mathfrak{m}_{R}\right)$, after finitely many iterations of the algorithm we obtain an expression (2.27) with $\rho \geq c v\left(\mathfrak{m}_{R}\right)$, which is a contradiction to (2.28). This contradiction shows that $P_{\Omega}$ is a unit times $g$, so we may replace $g$ with $P_{\Omega}$, and we are in Case 2 of the conclusions of the corollary.

If $\Omega=\infty$ then $v\left(P_{i}\right) \in \mathbb{Q} v\left(\mathfrak{m}_{R}\right)$ for all $i$ (by Remark 2.2.6) and we are in Case 3 of the conclusions of the corollary. 
The conclusions of a) and b) of Cases 2 and 3 of the corollary now follow from applying Theorem 2.2.11 and 2), 3) of Theorem 2.2.3 to $u$ in (2.26).

Suppose that $\lambda \in V_{v} / \mathfrak{m}_{v}$. Then $\lambda=\left[\frac{f}{f^{\prime}}\right]$ for some $f, f^{\prime} \in R$ with $v(f)=v\left(f^{\prime}\right)$. We may assume (after possibly dividing out a common factor) that $g \backslash f$ and $g \backslash f^{\prime}$. Then the proof of c) of cases 2 and 3 proceeds as in the proof of c) of Theorem 2.2.12.

\subsection{Valuation Semigroups and Residue Field Extension on a Two Dimensional Regular Local Ring}

In this section, we prove Theorem 2.3.1 which is stated below. Theorem 2.3.1 gives necessary and sufficient conditions for a semigroup and field extension to be the valuation semigroup and residue field of a valuation dominating a complete regular local ring of dimension two. If the regular local ring is not complete, those necessary conditions are provided in Corollary 2.3.2.

Theorem 2.3.1 Suppose that $R$ is a complete regular local ring of dimension two with

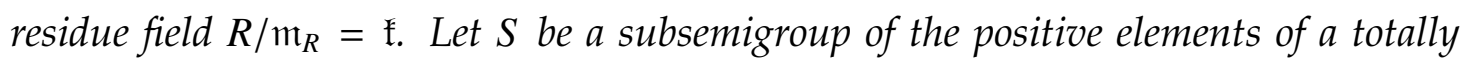
ordered abelian group and $L$ be a field extension of $f$. Then $S$ is the semigroup of a valuation $v$ dominating $R$ with residue field $V_{v} / \mathrm{m}_{v}=L$ if and only if there exists a finite or countable index set $I$, of cardinality $\Lambda=|I|-1 \geq 1$ and elements $\beta_{i} \in S$ for $i \in I$ and $\alpha_{i} \in L$ for $i \in I_{+}$, where $I_{+}=\{i \in I \mid i>0\}$, such that

1) The semigroup $S$ is generated by $\left\{\beta_{i}\right\}_{i \in I}$ and the field $L$ is generated over $¥$ by $\left\{\alpha_{i}\right\}_{i \in I_{+}}$. 
2) Let

$$
\bar{n}_{i}=\left[G\left(\beta_{0}, \ldots, \beta_{i}\right): G\left(\beta_{0}, \ldots, \beta_{i-1}\right)\right]
$$

and

$$
d_{i}=\left[\mathfrak{f}\left(\alpha_{1}, \ldots, \alpha_{i}\right): \mathfrak{f}\left(\alpha_{1}, \ldots, \alpha_{i-1}\right)\right] .
$$

Then there are inequalities

$$
\beta_{i+1}>\bar{n}_{i} d_{i} \beta_{i}>\beta_{i}
$$

with $\bar{n}_{i}<\infty$ and $d_{i}<\infty$ for $1 \leq i<\Lambda$ and if $\Lambda<\infty$, then either $\bar{n}_{\Lambda}=\infty$ and $d_{\Lambda}=1$ or $\bar{n}_{\Lambda}<\infty$ and $d_{\Lambda}=\infty$.

Proof Suppose that $v$ is a valuation dominating $R$. Let $S=S^{R}(v)$ and $L=V_{v} / \mathrm{m}_{v}$. Let $x, y$ be regular parameters in $R$ such that $v(x)=v\left(\mathrm{~m}_{R}\right)$. Set $P_{0}=x$ and $P_{1}=y$. Let $\left\{P_{i}\right\}$ be the sequence of elements of $R$ defined by the algorithm of Theorem 2.2.3. We have by Remark 2.2 .7 and its proof, that if

$$
\Omega=\infty \text { and } n_{i}=1 \text { for } i \gg 0,
$$

then $I_{\hat{R}} \neq(0)$ (where $I_{\hat{R}}$ is the prime ideal in $\hat{R}$ of Cauchy sequences in $R$ satisfying (2.2)). Thus $v$ has rank 2 since $R$ is complete, and $v$ must satisfy Case 3 of Theorem 2.2.13.

Set $\sigma(0)=0$ and inductively define

$$
\sigma(i)=\min \left\{j \mid j>\sigma(i-1) \text { and } n_{j}>1\right\}
$$

This defines an index set $I$ of finite or infinite cardinality $\Lambda=|I|-1 \geq 1$. Suppose that either $v$ has rank 1 or $v$ has rank 2 and one of the first two cases of Theorem 2.2.13 hold for the $P_{i}$. Let

$$
\beta_{i}=v\left(P_{\sigma(i)}\right) \in S^{R}(v)
$$


for $i \in I$ and

$$
\gamma_{i}=\left[\frac{P_{\sigma(i)}^{\bar{n}_{\sigma(i)}}}{U_{\sigma(i)}}\right] \in V_{v} / \mathrm{m}_{v}
$$

if $i>0$ and $\sigma(i)<\Omega$ or $\sigma(i)=\Omega$ and $\bar{n}_{\Omega}<\infty$. Set $\gamma_{\Lambda}=1$ if $\sigma(\Lambda)=\Omega$ and $\bar{n}_{\Omega}=\infty$.

By Theorem 2.2.3 and Theorem 2.2.12 or 2.2.13, $\left\{\beta_{i}\right\}$ and $\left\{\gamma_{i}\right\}$ satisfy the conditions 1) and 2) of Theorem 2.3.1.

Suppose that $v$ has rank 2 and the third case of Theorem 2.2.13 holds for the $P_{i}$. Then $\Lambda<\infty$. Let $I_{v} \cap R=(g)$ (where $I_{v}$ is the height one prime ideal of $\left.V_{v}\right)$. Let $\bar{\Lambda}=\Lambda+1$. Define $\beta_{i}=v\left(P_{\sigma(i)}\right)$ for $i<\bar{\Lambda}$ and $\beta_{\bar{\Lambda}}=v(g)$. Define

$$
\gamma_{i}=\left[\frac{P_{\sigma(i)}^{\bar{n}_{\sigma(i)}}}{U_{\sigma(i)}}\right] \in V_{v} / \mathrm{m}_{v}
$$

for $0<i<\bar{\Lambda}$ and define $\gamma_{\bar{\Lambda}}=1$. By Theorem 2.2.3 and Case 3 of Theorem 2.2.13, $\left\{\beta_{i}\right\}$ and $\left\{\gamma_{i}\right\}$ satisfy conditions 1 ) and 2) of Theorem 2.3.1.

Now suppose that $S$ and $L$ and the given sets $\left\{\beta_{i}\right\}$ and $\left\{\alpha_{i}\right\}$ satisfy conditions 1) and 2) of the theorem. We will construct a valuation $v$ which dominates $R$ with $S^{R}(v)=S$ and $V_{v} / \mathrm{m}_{v}=L$.

Let

$$
f_{i}(u)=u^{d_{i}}+b_{i, d_{i}-1} u^{d_{i}-1}+\cdots+b_{i, 0}
$$

be the minimal polynomial of $\alpha_{i}$ over $\mathfrak{f}\left(\alpha_{1}, \ldots, \alpha_{i-1}\right)$, and let $n_{i}=\bar{n}_{i} d_{i}$.

We will inductively define $P_{i} \in R$, a function $v$ on Laurent monomials in $P_{0}, \ldots, P_{i}$ such that

$$
v\left(P_{0}^{a_{0}} P_{1}^{a_{1}} \cdots P_{i}^{a_{i}}\right)=a_{0} \beta_{0}+a_{1} \beta_{1}+\cdots+a_{i} \beta_{i}
$$

for $a_{0}, \ldots, a_{i} \in \mathbb{Z}$ and monomials $U_{i}$ in $P_{0}, \ldots, P_{i-1}$, such that

$$
v\left(U_{i}\right)=\bar{n}_{i} \beta_{i},
$$


a function res on the Laurent monomials $P_{0}^{a_{0}} P_{1}^{a_{1}} \cdots P_{i}^{a_{i}}$ which satisfy $v\left(P_{0}^{a_{0}} P_{1}^{a_{1}} \cdots P_{i}^{a_{i}}\right)=0$, such that

$$
\operatorname{res}\left(\frac{P_{j}^{\bar{n}_{j}}}{U_{j}}\right)=\alpha_{j}
$$

for $1 \leq j \leq i$.

Let $x, y$ be regular parameters in $R$. Define $P_{0}=x, P_{1}=y, \beta_{0}=v\left(P_{0}\right)$, and $\beta_{1}=v\left(P_{1}\right)$. We inductively construct the $P_{i}$ by the procedure of the algorithm of Theorem 2.2.3. We must modify the inductive statement $A(i)$ of the proof of Theorem 2.2.3 as follows:

There exists $U_{i}=P_{0}^{w_{0}(i)} P_{1}^{w_{1}(i)} \cdots P_{i-1}^{w_{i-1}(i)}$ for some $w_{j}(i) \in \mathbb{N}$

and $0 \leq w_{j}(i)<n_{j}$ for $1 \leq j \leq i-1$

$\bar{A}(i) \quad$ such that $\bar{n}_{i} v\left(P_{i}\right)=v\left(U_{i}\right)$. There exist $a_{s, t} \in C S$

and $j_{0}(s, t), j_{1}(s, t), \ldots, j_{i-1}(s, t) \in \mathbb{N}$ with $0 \leq j_{k}(s, t)<n_{k}$

for $k \geq 1$ and $0 \leq t<\bar{d}_{i}$ such that

$v\left(P_{0}^{j_{0}(s, t)} P_{1}^{j_{1}(s, t)} \cdots P_{i-1}^{j_{i-1}(s, t)} P_{i}^{t \bar{n}_{i}}\right)=\bar{n}_{i} d_{i} v\left(P_{i}\right)$

for all $s, t$ and

$$
P_{i+1}:=P_{i}^{\bar{n}_{i} d_{i}}+\sum_{t=0}^{d_{i}-1}\left(\sum_{s=1}^{\lambda_{t}} a_{s, t} P_{0}^{j_{0}(s, t)} P_{1}^{j_{1}(s, t)} \cdots P_{i-1}^{j_{i-1}(s, t)}\right) P_{i}^{t \bar{n}_{i}}
$$

satisfies

$$
\begin{aligned}
& \qquad b_{i, t}=\sum_{s=1}^{\lambda_{t}} \bar{a}_{s, t} \operatorname{res}\left(\frac{P_{0}^{j_{0}(s, t)} P_{1}^{j_{1}(s, t)} \ldots P_{i-1}^{j_{i-1}(s, t)}}{U_{i}^{d_{i}-t}}\right) \\
& \text { for } 0 \leq t \leq d_{i}-1 .
\end{aligned}
$$

We inductively verify $\bar{A}(i)$ for $1 \leq i<\Lambda$ and the statements $B(i), C(i)$ and $D(i)$ (with the residues $[M]$ replaced with res $(M)$ ). We observe from $B(i)$ that the 
function res is determined by (2.31). The inequality in 2) of the assumptions of the theorem is necessary to allow us to apply Lemma 2.2.2.

We now show that if $\Lambda=\infty$, then given $\sigma \in \mathbb{Z}_{+}$, there exists $\tau \in \mathbb{Z}_{+}$such that

$$
\operatorname{ord}\left(P_{i}\right)>\sigma \text { if } i>\tau \text {. }
$$

We establish (2.33) by induction on $\sigma$. Suppose that $\operatorname{ord}\left(P_{i}\right)>\sigma$ if $i>\tau$. There exists $\lambda$ such that $\beta_{0}<\beta_{i}$ if $i \geq \lambda$. Let $\tau^{\prime}=\max \{\sigma+\tau+1, \tau+1, \lambda\}$. We will show that $\operatorname{ord}\left(P_{i}\right)>\sigma+1$ if $i>\tau^{\prime}$. From (2.32), we must show that if $i>\tau^{\prime}$ and $\left(a_{0}, \ldots, a_{i-1}\right) \in \mathbb{N}^{i}$ are such that

$$
a_{0} \beta_{0}+a_{1} \beta_{1}+\cdots+a_{i-1} \beta_{i-1}=n_{i-1} \beta_{i-1}
$$

then

$$
a_{0} \operatorname{ord}\left(P_{0}\right)+a_{1} \operatorname{ord}\left(P_{1}\right)+\cdots+a_{i-1} \operatorname{ord}\left(P_{i-1}\right)>\sigma+1 .
$$

If $a_{\tau+1}+\cdots+a_{i-1} \geq 2$ then (2.34) follows from induction. If $a_{\tau+1}+\cdots+a_{i-1}=1$ then some $a_{j} \neq 0$ with $0 \leq j \leq \tau$ since $n_{i-1}>1$, so (2.34) follows from induction. If $a_{j}=0$ for $j \geq \tau+1$ then

$$
n_{i-1} \beta_{i-1}=a_{0} \beta_{0}+\cdots+a_{\tau} \beta_{\tau}<\left(a_{0}+\cdots+a_{\tau}\right) \beta_{\tau} .
$$

Thus

$$
\left(a_{0}+\cdots+a_{\tau}\right)>\frac{n_{i-1} \beta_{i-1}}{\beta_{\tau}} \geq 2^{i-\tau}>\sigma+1 .
$$

Thus (2.34) holds in this case.

We first suppose that for all $P_{i}$, there exists $m_{i} \in \mathbb{Z}_{+}$such that $m_{i} v\left(P_{i}\right)>$ $\min \left\{\beta_{0}, \beta_{1}\right\}$.

We now establish the following: 
Suppose that $f \in R$. Then there exists an expansion

$$
f=\sum_{I} a_{I} P_{0}^{i_{0}} P_{1}^{i_{1}} \cdots P_{r}^{i_{r}}+\sum_{J} \phi_{J} P_{0}^{j_{0}} \cdots P_{r}^{j_{r}}
$$

for some $r \in \mathbb{N}$ where $v\left(P_{0}^{i_{0}} P_{1}^{i_{1}} \ldots P_{r}^{i_{r}}\right)$ have a common value $\rho$ for all terms in the first sum, all $a_{I} \in C S, I, J \in \mathbb{N}^{r+1}$ and some $a_{I} \neq 0,0 \leq i_{k}<n_{k}$ for $1 \leq k \leq r v\left(P_{0}^{j_{0}} \cdots P_{r}^{j_{r}}\right)>\rho$ for all terms in the second sum, and $\phi_{J} \in R$ for all terms in the second sum. The first sum $\sum_{I} a_{I} P_{0}^{i_{0}} P_{1}^{i_{1}} \ldots P_{r}^{i_{r}}$ is uniquely determined by these conditions.

The proof of (2.35) follows from the proofs of Lemma 2.2.10 and Theorem 2.2.11, observing that all properties of a valuation which $v$ is required to satisfy in these proofs hold for the function $v$ on Laurent monomials in the $P_{i}$ which we have defined above, and replacing $[M]$ in Lemma 2.2.10 with the function $\operatorname{res}(M)$ for Laurent monomials $M$ with $v(M)=0$.

The $n$ in the statement of Theorem 2.2.11 is chosen so that if $M$ is a monomial in the $P_{i}$ with $\operatorname{ord}(M)=\operatorname{ord}(f)$, then $v(M)<n \min \left\{\beta_{0}, \beta_{1}\right\}$ (such an $n$ exists trivially if $\Lambda<\infty$ and by (2.33) if $\Lambda=\infty)$.

We can thus extend $v$ to $R$ by defining

$$
v(f)=\rho \text { if } f \text { has an expansion (2.35). }
$$

Now we will show that $v$ is a valuation. Suppose that $f, g \in R$. We have expansions

$$
f=\sum_{I} a_{I} P_{0}^{i_{0}} P_{1}^{i_{1}} \cdots P_{r}^{i_{r}}+\sum_{J} \phi_{J} P_{0}^{j_{0}} \cdots P_{r}^{j_{r}}
$$

and

$$
g=\sum_{K} b_{K} P_{0}^{k_{0}} P_{1}^{k_{1}} \cdots P_{r}^{k_{r}}+\sum_{L} \phi_{L} P_{0}^{l_{0}} \cdots P_{r}^{l_{r}}
$$

of the form (2.35). Let $\rho=v(f)$ and $\rho^{\prime}=v(g)$. The statement that $v(f+g) \geq$ $\min \{v(f), v(g)\}$ follows from Remark 2.2.1 and the algorithm of Theorem 2.2.11. 
Let $V$ be a monomial in $P_{0}, \ldots, P_{r}$ such that $v(V)=v\left(P_{0}^{i_{0}} \cdots P_{r}^{i_{r}}\right)$ for all $I$ in the first sum of $f$ in (2.36) and let $W$ be a monomial in $P_{0}, \ldots, P_{r}$ such that $v(W)=$ $v\left(P_{0}^{k_{0}} \cdots P_{r}^{k_{r}}\right)$ for all $K$ in the first sum of $g$ in (2.37). We have that

$$
\sum \bar{a}_{I} \operatorname{res}\left(\frac{P_{0}^{i_{0}} \cdots P_{r}^{i_{r}}}{V}\right) \neq 0 \text { in } L
$$

and

$$
\sum \bar{b}_{K} \operatorname{res}\left(\frac{P_{0}^{k_{0}} \cdots P_{r}^{k_{r}}}{W}\right) \neq 0 \text { in } L
$$

by $D(r)$.

We have (applying Remark 2.2.1) an expansion

$$
f g=\sum_{M} d_{M} P_{0}^{m_{0}} P_{1}^{m_{1}} \cdots P_{r}^{m_{r}}+\sum_{Q} \psi_{Q} P_{0}^{q_{0}} \cdots P_{r}^{q_{r}}
$$

with $d_{M} \in S$ for all $M, \psi_{Q} \in R$ for all $Q, v\left(P_{0}^{m_{0}} P_{1}^{m_{1}} \cdots P_{s}^{m_{r}}\right)=\rho+\rho^{\prime}$ for all terms in the first sum, and some $d_{M} \neq 0$ and $v\left(P_{0}^{q_{0}} \cdots P_{r}^{q_{r}}\right)>\rho+\rho^{\prime}$ for all terms in the second sum, which satisfies all conditions of (2.35) except that we only have that $m_{0}, m_{1}, \ldots, m_{r} \in \mathbb{N}$. We have

$$
\sum_{M} \bar{d}_{M} \operatorname{res}\left(\frac{P_{0}^{m_{0}} \cdots P_{r}^{m_{r}}}{V W}\right)=\left(\sum_{I} \bar{a}_{I} \operatorname{res}\left(\frac{P_{0}^{i_{0}} \cdots P_{r}^{i_{r}}}{V}\right)\right)\left(\sum_{K} \bar{b}_{K} \operatorname{res}\left(\frac{P_{0}^{k_{0}} \cdots P_{r}^{k_{r}}}{W}\right)\right) \neq 0 .
$$

By (2.20) of Lemma 2.2.10 (with $[M]$ replaced with res $(M)$ for a Laurent monomial $N$ with $v(M)=0$ ) we see that the algorithm of Theorem 2.2.11 which puts the expansion (2.38) into the form (2.35) converges to an expression (2.35) where the terms in the first sum all have $v\left(P_{0}^{i_{0}} \cdots P_{r}^{i_{r}}\right)=\rho+\rho^{\prime}$ with

$$
\sum_{I} \bar{a}_{I} \operatorname{res}\left(\frac{P_{0}^{i_{0}} \cdots P_{r}^{i_{r}}}{V W}\right)=\sum_{M} \bar{d}_{M} \operatorname{res}\left(\frac{P_{0}^{m_{0}} \cdots P_{r}^{m_{r}}}{V W}\right) \neq 0 .
$$

Thus $v(f g)=v(f)+v(g)$. We have established that $v$ is a valuation.

By Theorem 2.2.12 or Case 1 of Theorem 2.2.13, we have that $S=S^{R}(v)$ and $L=V_{v} / \mathfrak{m}_{v}$. 
Finally, we suppose that $\Lambda$ is finite and $\bar{n}_{\Lambda}=\infty$. Given $g \in R$, write

$$
g=P_{\Lambda}^{t} f
$$

where $P_{\Lambda} \not f$. Choose $n \in \mathbb{Z}_{+}$so that if $M$ is a monomial in $P_{0}, \ldots, P_{\Lambda-1}$ with $\operatorname{ord}(M)=\operatorname{ord}(f)$ then $v(M)<n \min \left\{\beta_{0}, \beta_{1}\right\}$.

The argument giving the expansion (2.35) now provides an expansion

$$
f=\sum_{I} a_{I} P_{0}^{i_{0}} \cdots P_{\Lambda}^{i_{\Lambda}}+\sum_{J} \phi_{J} P_{0}^{j_{0}} \cdots P_{\Lambda}^{j_{\Lambda}}+h_{1}
$$

where $v\left(P_{0}^{i_{0}} \cdots P_{\Lambda}^{i_{\Lambda}}\right)$ has a common value $\rho$ for all monomials in the first sum, $a_{I} \in C S$ for all $I, v\left(P_{0}^{j_{0}} \cdots P_{\Lambda}^{j_{\Lambda}}\right)>\rho$ for all monomials in the second sum, $\phi_{J} \in R$ for all $J$ and $h_{1} \in \mathfrak{m}_{R}^{n}$.

If $i_{\Lambda}=0$ for all monomials in the first sum, then we obtain an expansion of $f$ of the form (2.35). Suppose that $i_{\Lambda} \neq 0$ for some monomial in the first sum. Then $i_{\Lambda} \neq 0$ for all terms in the first sum, $j_{\Lambda} \neq 0$ for all terms in the second sum, and we have an expression $f=P_{\Lambda} t_{1}+h_{1}$ for some $t_{1} \in R$. Repeating this argument for increasingly large values of $n$, we either obtain an $n$ giving an expression (2.35) for $f$, or we obtain the statement that

$$
f \in \cap_{n=1}^{\infty}\left(\left(P_{\Lambda}\right)+\mathfrak{m}_{R}^{n}\right)=\left(P_{\Lambda}\right),
$$

which is impossible. Thus we can extend $v$ to $R$ by defining $v(g)=t \beta_{\Lambda}+\rho$ if $g=P_{\Lambda}^{t} f$ where $P_{\Lambda} \not f f$ and $f$ has an expansion (2.35).

It follows that $v$ is a valuation, by an extension of the proof of the previous case. By Case 2 of Theorem 2.2.13, we have that $S=S^{R}(v)$ and $L=V_{v} / \mathrm{m}_{v}$.

Corollary 2.3.2 Suppose that $R$ is a regular local ring of dimension two and $v$ is a valuation dominating $R$. Then the semigroup $S^{R}(v)$ has a generating set $\left\{\beta_{i}\right\}_{i \in I}$ and 
$V_{v} / \mathfrak{m}_{v}$ is generated over $\mathfrak{f}=R / \mathfrak{m}_{R}$ by a set $\left\{\alpha_{i}\right\}_{i \in I_{+}}$such that 1) and 2) of Theorem 2.3.1 hold, but the additional case that $\bar{n}_{\Lambda}<\infty$ and $d_{\Lambda}<\infty$ if $\Lambda<\infty$ may hold if $R$ is not complete.

Proof The only case we have not considered in Theorem 2.3.1 is the analysis in the case when $\Omega=\infty, n_{i}=1$ for $i \gg 0, I_{\hat{R}} \neq 0$ and $I_{\hat{R}} \cap R=(0)$ (so that $R$ is not complete). In this case $v$ is discrete of rank $1, \Lambda<\infty, \bar{n}_{\lambda}<\infty$ and $d_{\Lambda}<\infty$ by Remark 2.2.7, giving the additional possibility stated in the Corollary.

\subsection{Valuation Semigroups on a Regular Local Ring of Dimen- sion Two}

In this section we prove Theorem 2.4.1 which is stated below. Theorem 2.4.1 gives necessary and sufficent conditions for a semigroup to be the valuation semigroup of a valuation dominating a regular local ring of dimension two. In contrast to the case of complete regular local rings in Theorem 2.3.1, in Example 2.4.4 we construct a specific semigroup of valuations that allows no residue field extension.

Theorem 2.4.1 Suppose that $R$ is a regular local ring of dimension two. Let $S$ be a subsemigroup of the positive elements of a totally ordered abelian group. Then $S$ is the semigroup of a valuation $v$ dominating $R$ if and only if there exists a finite or countable index set $I$, of cardinality $\Lambda=|I|-1 \geq 1$ and elements $\beta_{i} \in S$ for $i \in I$ such that

1) The semigroup $S$ is generated by $\left\{\beta_{i}\right\}_{i \in I}$. 
2) Let

$$
\bar{n}_{i}=\left[G\left(\beta_{0}, \ldots, \beta_{i}\right): G\left(\beta_{0}, \ldots, \beta_{i-1}\right)\right] .
$$

There are inequalities

$$
\beta_{i+1}>\bar{n}_{i} \beta_{i}
$$

with $\bar{n}_{i}<\infty$ for $1 \leq i<\Lambda$. If $\Lambda<\infty$ then $\bar{n}_{\Lambda} \leq \infty$.

Proof If $S=S^{R}(v)$ for some valuation $v$ dominating $R$, then 1) and 2) of Theorem 2.4.1 hold by Corollary 2.3.2. Observe that the construction in the proof of Theorem 2.3.1 of a valuation $v$ with a prescribed semigroup $S$ and residue field $L$ satisfying the conditions 1 ) and 2) of Theorem 2.3.1 is valid for any regular local ring $R$ of dimension 2 (with residue field $\mathfrak{f}$ ). Taking $L=\mathfrak{f}$ (or $L=\mathfrak{f}(t)$ where $t$ is an indeterminate), we may thus construct a valuation $v$ dominating $R$ with semigroup $S^{R}(v)=S$ whenever $S$ satisfies the conditions 1) and 2) of Theorem 2.4.1.

Definition 2.4.2 Suppose that $S$ is a semigroup such that the group $G$ generated by $S$ is isomorphic to $\mathbb{Z}$. $S$ is symmetric if there exists $m \in G$ such that $s \in S$ if and only if $m-s \notin S$ for all $s \in G$.

We deduce from Theorem 2.4.1 a generalization of a result of Noh [39].

Corollary 2.4.3 Suppose that $R$ is a regular local ring of dimension two and $v$ is $a$ valuation dominating $R$ such that $v$ is discrete of rank 1 . Then $S^{R}(v)$ is symmetric.

Proof By Theorem 2.4.1, and since $v$ is discrete of rank 1, there exists a finite set

$$
\beta_{0}<\beta_{1}<\cdots<\beta_{\Lambda}
$$


such that $S^{R}(v)=S\left(\beta_{0}, \beta_{1}, \ldots, \beta_{\Lambda}\right)$ and $\beta_{i+1}>\bar{n}_{i} \beta_{i}$ for $1 \leq i<\Lambda$, where $\bar{n}_{i}=$ $\left[G\left(\beta_{0}, \ldots, \beta_{i}\right): G\left(\beta_{0}, \ldots, \beta_{i-1}\right)\right]$. We identify the value group $\Gamma_{v}$ with $\mathbb{Z}$. Then we calculate that

$$
\operatorname{lcm}\left(\operatorname{gcd}\left(\beta_{0}, \ldots, \beta_{i-1}\right), \beta_{i}\right)=\bar{n}_{i} \beta_{i}
$$

for $1 \leq i \leq \Lambda$. We have that $\bar{n}_{i} \beta_{i} \geq \beta_{i}>\bar{n}_{i-1} \beta_{i-1}$ for $2 \leq i \leq \Lambda$. By Lemma 2.2.2, we have that $\bar{n}_{i} \beta_{i} \in S\left(\beta_{0}, \ldots, \beta_{i-1}\right)$ for $2 \leq i \leq \Lambda$. Since $\beta_{0}$ and $\beta_{1}$ are both positive, we have that $\bar{n}_{1} \beta_{1} \in S\left(\beta_{0}\right)$. Thus the criteria of Proposition 2.1 [30] is satisfied, so that $S^{R}(v)$ is symmetric.

Example 2.4.4 There exists a semigroup $S$ which satisfies the sufficient conditions 1) and 2) of Theorem 2.4.1, such that if $\left(R, \mathrm{~m}_{R}\right)$ is a 2-dimensional regular local ring dominated by a valuation $v$ such that $S^{R}(v)=S$, then $R / \mathrm{m}_{R}=V_{v} / \mathrm{m}_{v}$; that is, there can be no residue field extension.

Proof Define $\beta_{i} \in \mathbb{Q}$ by

$$
\beta_{0}=1, \beta_{1}=\frac{3}{2}, \text { and } \beta_{i}=2 \beta_{i-1}+\frac{1}{2^{i}} \text { for } i \geq 2 .
$$

Let $S=S\left(\beta_{0}, \beta_{1}, \ldots\right)$ be the semigroup generated by $\beta_{0}, \beta_{1}, \ldots$. Observe that $\bar{n}_{i}=2, \forall i \geq 1, \beta_{0}<\beta_{1}<\cdots$ is the minimal sequence of generators of $S$ and $S$ satisfies conditions 1) and 2) of Theorem 2.4.1. The group $\Gamma=G\left(\beta_{0}, \beta_{1}, \ldots\right)$ generated by $S$ is $\Gamma=\frac{1}{2^{\infty}} \mathbb{Z}=\cup_{i=0}^{\infty} \frac{1}{2^{i}} \mathbb{Z}$.

Now suppose that $\left(R, \mathfrak{m}_{R}\right)$ is a regular local ring of dimension 2 , with residue field $\mathfrak{f}$ and $v$ is a valuation of the quotient field of $R$ which dominates $R$ such that $S^{R}(v)=S$. Since $\Gamma_{v}=\frac{1}{2^{\infty}}$ is not discrete, we have by Proposition 2.1.4 that $v$ extends uniquely to a valuation $\hat{v}$ of the quotient field of $\hat{R}$ which dominates $\hat{R}$ and $S^{\hat{R}}(\hat{v})=S$. 
We will now show that $V_{v} / \mathfrak{m}_{v}=V_{\hat{v}} / \mathfrak{m}_{\hat{v}}$. Suppose that $f \in \hat{R}$. Since $\hat{v}$ has rank 1 , there exists a positive integer $n$ such that $\hat{v}(f)<n v(\mathfrak{m})$. There exists $f^{\prime} \in R$ such that $f^{\prime \prime}=f-f^{\prime} \in \mathfrak{m}_{R}^{n} \hat{R}$. Thus $v(f)=v\left(f^{\prime}\right)$. Suppose that $h \in V_{\hat{v}} / \mathfrak{m}_{\hat{v}}$. Then $h=\left[\frac{f}{g}\right]$ where $f, g \in \hat{R}$ and $v(f)=v(g)$. Write $f=f^{\prime}+f^{\prime \prime}$ and $g=g^{\prime}+g^{\prime \prime}$ where $f^{\prime}, g^{\prime} \in R$ and $f^{\prime \prime}, g^{\prime \prime} \in \hat{R}$ satisfy $v\left(f^{\prime \prime}\right)>v(f)$ and $v\left(g^{\prime \prime}\right)>v(g)$. Then $\left[\frac{f}{g}\right]=\left[\frac{f^{\prime}}{g^{\prime}}\right] \in V_{v} / \mathfrak{m}_{v}$.

We also have $\mathfrak{f}=R / \mathfrak{m}_{R}=\hat{R} / \mathfrak{m}_{\hat{R}}$. By Theorem 2.3.1, there exists $\alpha_{i} \in V_{\hat{v} / \mathfrak{m}_{\hat{v}}}$ for $i \geq 1$ such that $V_{\hat{v} / m_{\hat{v}}}=\mathfrak{f}\left(\alpha_{1}, \alpha_{2}, \ldots\right)$ and if $d_{i}=\left[\mathfrak{f}\left(\alpha_{1}, \ldots, \alpha_{i}\right): \mathfrak{f}\left(\alpha_{1}, \ldots, \alpha_{i-1}\right)\right]$ then

$$
\beta_{i+1} \geq \bar{n}_{i} d_{i} \beta_{i}, \forall i \geq 1
$$

so that

$$
\left[V_{\hat{v}} / \mathfrak{m}_{\hat{v}}: \mathfrak{f}\right]=\prod_{i=1}^{\infty}\left[\mathfrak{F}\left(\alpha_{1}, \ldots, \alpha_{i}\right): \mathfrak{f}\left(\alpha_{1}, \ldots, \alpha_{i-1}\right)\right]=\prod_{i=1}^{\infty} d_{i} .
$$

On the other hand, since $\beta_{i} \geq \beta_{1}=\frac{3}{2}, \forall i \geq 1$, we have

$$
\beta_{i+1}=2 \beta_{i}+\frac{1}{2^{i+1}} \leq 4 \beta_{i}+\frac{1}{2^{i+1}}-3<4 \beta_{i} .
$$

From (2.42), (2.43) and (2.44) we have $d_{i}=1, \forall i \geq 1$ so that $\left[V_{\hat{v}} / \mathfrak{m}_{\hat{v}}: \mathfrak{f}\right]=1$.

\subsection{Birational Extensions}

Suppose that $R$ is a regular local ring of dimension two which is dominated by a valuation $v$. Let $\mathfrak{f}=R / \mathrm{m}_{R}$. The quadratic transform $T_{1}$ of $R$ along $v$ is defined as follows. Let $u, v$ be a system of regular parameters in $R$, where we may assume that $v(u) \leq v(v)$. Then $R\left[\frac{v}{u}\right] \subset V_{v}$. Let

$$
T_{1}=R\left[\frac{v}{u}\right]_{R\left[\frac{v}{u}\right] \cap m_{v}} .
$$

$T_{1}$ is a two dimensional regular local ring which is dominated by $v$. Let

$$
R \rightarrow T_{1} \rightarrow T_{2} \cdots
$$


be the sequence of quadratic transforms along $v$, so that $V_{v}=U T_{i}$ ([1]), and $L=V_{v} / \mathfrak{m}_{v}=\cup T_{i} / \mathfrak{m}_{T_{i}}$. Suppose that $x, y$ are regular parameters in $R$.

Theorem 2.5.1 Let $P_{0}=x, P_{1}=y$ and $\left\{P_{i}\right\}$ be the sequence of elements of $R$ constructed in Theorem 2.2.3. Suppose that $\Omega \geq 2$. Then there exists some smallest value $i$ in the sequence (2.45) such that the divisor of $x y$ in $\operatorname{Spec}\left(T_{i}\right)$ has only one component. Let $R_{1}=T_{i}$. Then $R_{1} / \mathrm{m}_{R_{1}} \cong \mathfrak{f}\left(\alpha_{1}\right)$, and there exists $x_{1} \in R_{1}$ and $w \in \mathbb{Z}_{+}$such that $x_{1}=0$ is a local equation of the exceptional divisor of $\operatorname{Spec}\left(R_{1}\right) \rightarrow \operatorname{Spec}(R)$, and $Q_{0}=x_{1}, Q_{1}=\frac{P_{2}}{x_{1}^{\text {xpl }}}$ are regular parameters in $R_{1}$. We have that

$$
Q_{i}=\frac{P_{i+1}}{Q_{0}^{w_{1} \cdots n_{i}}}
$$

for $1 \leq i<\max \{\Omega, \infty\}$ satisfy the conclusions of Theorem 2.2.3 (as interpreted by Remark 2.2.4) for the ring $R_{1}$.

Proof We use the notation of Theorem 2.2.3 and its proof for $R$ and the $\left\{P_{i}\right\}$. Recall that $U_{1}=x^{w_{0}(1)}$. Let $w=w_{0}(1)$. Since $\bar{n}_{1}$ and $w$ are relatively prime, there exist $a, b \in \mathbb{N}$ such that

$$
\epsilon:=\bar{n}_{1} b-w a= \pm 1 \text {. }
$$

Define elements of the quotient field of $R$ by

$$
x_{1}=\left(x^{b} y^{-a}\right)^{\epsilon}, y_{1}=\left(x^{-w} y^{\bar{n}_{1}}\right)^{\epsilon} .
$$

We have that

$$
x=x_{1}^{\bar{n}_{1}} y_{1}^{a}, y=x_{1}^{w} y_{1}^{b} .
$$

Since $\bar{n}_{1} v(y)=w v(x)$, it follows that

$$
\bar{n}_{1} v\left(x_{1}\right)=v(x), \bar{v}\left(y_{1}\right)=0 .
$$


We further have that

$$
\alpha_{1}=\left[y_{1}\right]^{\epsilon} \in L
$$

Let $A=R\left[x_{1}, y_{1}\right] \subset V_{v}$ and $\mathrm{m}_{A}=\mathfrak{m}_{v} \cap A . R \rightarrow A_{\mathfrak{m}_{A}}$ factors as a product of quadratic transforms such that $x y$ has two distinct irreducible factors in all intermediate rings. Thus $A=R_{1}$. Recall that

$$
f_{1}(u)=u^{d_{1}}+b_{1, d_{1}-1} u^{d_{1}-1}+\cdots+b_{1,0}
$$

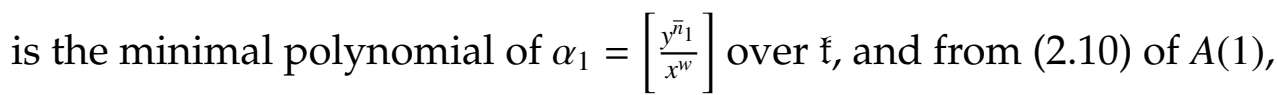

$$
P_{2}=y^{\bar{n}_{1} d_{1}}+a_{1, d_{1}-1} x^{w} y^{\bar{n}_{1}\left(d_{1}-1\right)}+\cdots+a_{1,0} x^{d_{1} w} .
$$

Substituting (2.46) into (2.48), we find that

$$
P_{2}=x_{1}^{w n_{1}}\left(y_{1}^{b \bar{n}_{1} d_{1}}+a_{1, d_{1}-1} y_{1}^{a w+b \bar{n}_{1}\left(d_{1}-1\right)}+\cdots+a_{1,0} y_{1}^{a d_{1} w}\right) .
$$

Thus

$$
Q_{1}=\frac{P_{2}}{x_{1}^{w n_{1}}} \in R_{1}
$$

We calculate

$$
v\left(Q_{1}\right)=v\left(P_{2}\right)-w n_{1} v\left(x_{1}\right)=v\left(P_{2}\right)-n_{1} v\left(P_{1}\right)>0
$$

Thus $x_{1}, Q_{1} \in \mathfrak{m}_{R_{1}}$.

Suppose that $\epsilon=1$. Then since

$$
Q_{1}=y_{1}^{a w d_{1}}\left(y_{1}^{d_{1}}+a_{1, d_{1}-1} y_{1}^{d_{1}-1}+\cdots+a_{1,0}\right)
$$

and $y_{1}$ is a unit in $R_{1}$, we have that

$$
R_{1} /\left(x_{1}, Q_{1}\right) \cong \mathfrak{f}\left[y_{1}\right] /\left(f\left(y_{1}\right)\right) \cong \mathfrak{f}\left(\alpha_{1}\right) .
$$

Suppose that $\epsilon=-1$. Let

$$
h(u)=y_{1}^{d_{1}}+\frac{b_{1,1}}{b_{1,0}} y_{1}^{d_{1}-1}+\cdots+\frac{1}{b_{1,0}},
$$


which is the minimal polynomial of $\alpha_{1}^{-1}$ over f. Since

$$
Q_{1}=y_{1}^{b \bar{n}_{1} d_{1}}\left(1+a_{1, d_{1}-1} y_{1}+\cdots+a_{1,0} y_{1}^{d_{1}}\right)
$$

and $y_{1}$ is a unit in $R_{1}$, we have that

$$
R_{1} /\left(x_{1}, Q_{1}\right) \cong \mathfrak{f}\left[y_{1}\right] /\left(h\left(y_{1}\right)\right) \cong \mathfrak{f}\left(\alpha_{1}^{-1}\right)=\mathfrak{f}\left(\alpha_{1}\right) .
$$

Now define $\beta_{i}=v\left(P_{i}\right)$ and $\hat{\beta}_{i}=v\left(Q_{i}\right)$ for $i \geq 0$. We have

$$
\hat{\beta}_{i}=v\left(P_{i+1}\right)-w n_{1} \cdots n_{i} \hat{\beta}_{0}
$$

for $i \geq 1$.

Since $\operatorname{gcd}\left(w, \bar{n}_{1}\right)=1$, we have that $G\left(\hat{\beta}_{0}\right)=G\left(\beta_{0}, \beta_{1}\right)$. Thus

$$
\bar{n}_{i+1}=\left[G\left(\hat{\beta}_{0}, \ldots, \hat{\beta}_{i}\right): G\left(\hat{\beta}_{0}, \ldots, \hat{\beta}_{i-1}\right)\right]
$$

for $i \geq 1$.

We will leave the proof that the analogue of $A(1)$ of Theorem 2.2.3 holds for $Q_{1}$ in $R_{1}$ for the reader, as is an easier variation of the following inductive statement, which we will prove.

Assume that $2 \leq i<\Omega-1$ and the analogue of $A(j)$ of Theorem 2.2.3 holds for $Q_{j}$ in $R_{1}$ for $j<i$. We will prove that the analogue of $A(i)$ of Theorem 2.2.3 holds for $Q_{i}$ in $R_{1}$.

In particular, we assume that

$$
\hat{\beta}_{j+1}>n_{j+1} \hat{\beta}_{j}
$$

for $1 \leq j \leq i-1$. 
Define

$$
\begin{aligned}
V_{i} & =U_{i+1} Q_{0}^{-w n_{1} n_{2} \cdots n_{i} \bar{n}_{i+1}} y_{1}^{-\left(a w_{0}(i+1)+b w_{1}(i+1)\right)} \\
& =Q_{0}^{\hat{w}_{0}(i+1)} Q_{1}^{w_{2}(i+1)} \cdots Q_{i-1}^{w_{i}(i+1)}
\end{aligned}
$$

where

$\hat{w}_{0}(i+1)=\bar{n}_{1} w_{0}(i+1)+w w_{1}(i+1)+w n_{1} w_{2}(i+1)+\cdots+w n_{1} n_{2} \cdots n_{i-1} w_{i}(i+1)-w n_{1} n_{2} \cdots n_{i} \bar{n}_{i+1}$.

We have that

$$
v\left(Q_{i}^{\bar{n}_{i+1}}\right)=\bar{n}_{i+1} \hat{\beta}_{i}=\bar{n}_{i+1} v\left(P_{i+1}\right)-w n_{1} n_{2} \cdots n_{i} \bar{n}_{i+1} \hat{\beta}_{0}=v\left(V_{i}\right) .
$$

Thus

$$
\bar{n}_{i+1} \hat{\beta}_{i}=\hat{w}_{0}(i+1) \hat{\beta}_{0}+\hat{w}_{2}(i+1) \hat{\beta}_{1}+\hat{w}_{3}(i+1) \hat{\beta}_{2}+\cdots+w_{i}(i+1) \hat{\beta}_{i-1} .
$$

Recall that $0 \leq w_{j}(i+1)<n_{j}$ for $1 \leq j \leq i$ and apply (2.52) to obtain

$$
\begin{aligned}
\hat{w}_{0}(i+1) \hat{\beta}_{0}= & \bar{n}_{i+1} \hat{\beta}_{i}-w_{i}(i+1) \hat{\beta}_{i-1}-\cdots-w_{3}(i+1) \hat{\beta}_{2}-w_{2}(i+1) \hat{\beta}_{1} \\
\geq & \hat{\beta}_{i}-\left(n_{i}-1\right) \hat{\beta}_{i-1}-\cdots-\left(n_{3}-1\right) \hat{\beta}_{2}-\left(n_{2}-1\right) \hat{\beta}_{1} \\
> & \hat{\beta}_{i}-\left(n_{i}-1\right) \hat{\beta}_{i-1}-\cdots-\left(n_{4}-1\right) \hat{\beta}_{3}-n_{3} \hat{\beta}_{2} \\
& \vdots \\
\geq & \hat{\beta}_{i}-n_{i} \hat{\beta}_{i-1}>0 .
\end{aligned}
$$

Thus $V_{i} \in R_{1}$. We have

$$
\frac{Q_{i}^{\bar{n}_{i+1}}}{V_{i}}=\left(\frac{P_{i+1}^{\bar{n}_{i+1}}}{U_{i+1}}\right) y_{1}^{a w_{0}(i+1)+b w_{1}(i+1)} .
$$

Let

$$
\hat{\alpha}_{i}=\left[\frac{Q_{i}^{\bar{n}_{i+1}}}{V_{i}}\right]=\alpha_{i+1} \alpha_{1}^{\epsilon\left(a w_{0}(i+1)+b w_{1}(i+1)\right)} \in L
$$

From the minimal polynomial $f_{i+1}(u)$ of $\alpha_{i+1}$, we see that

$$
g_{i}(u)=u^{d_{i+1}}+b_{i+1, d_{i+1}-1} \alpha_{1}^{\epsilon\left(a w_{0}(i+1)+b w_{1}(i+1)\right) d_{i+1}} u^{d_{i+1}-1}+\cdots+b_{i+1,0} \alpha_{1}^{\epsilon\left(a w_{0}(i+1)+b w_{1}(i+1)\right) d_{i+1}}
$$

is the minimal polynomial of $\hat{\alpha}_{i}$ over $\mathfrak{f}\left(\alpha_{1}\right)\left(\hat{\alpha}_{1}, \ldots, \hat{\alpha}_{i-1}\right)$. 
Now from equation (2.10) of $A(i+1)$ determining $P_{i+1}$, we obtain

$$
\begin{aligned}
Q_{i+1} & =\frac{P_{i+2}}{Q_{0}^{w n n_{1} n^{2} n_{i+1}}} \\
& =Q_{i}^{\bar{n}_{i+1} d_{i+1}}+\sum_{t=0}^{d_{i+1}-1}\left(\sum_{s=1}^{\lambda_{t}} a_{s, t} y_{1}^{a j_{0}(s, t)+b j_{1}(s, t)} Q_{0}^{\hat{j}_{0}(s, t)} Q_{1}^{j_{2}(s, t)} \cdots Q_{i-1}^{j_{i}(s, t)}\right) Q_{i}^{t \bar{n}_{i+1}}
\end{aligned}
$$

where

$\hat{j}_{0}(s, t)=\bar{n}_{1} j_{0}(s, t)+w j_{1}(s, t)+w n_{1} j_{2}(s, t)+\cdots+w n_{1} n_{2} \cdots n_{i-1} j_{i}(s, t)-\left(d_{i+1}-t\right) w n_{1} n_{2} \cdots n_{i} \bar{n}_{i+1}$.

Recall that $0 \leq j_{k}(s, t)<n_{k}$ for $1 \leq k \leq i$. We further have that

$$
v\left(Q_{0}^{\hat{j}_{0}(s, t)} Q_{1}^{j_{2}(s, t)} \cdots Q_{i-1}^{j_{i}(s, t)}\right)=\left(d_{i+1}-t\right) \bar{n}_{i+1} \hat{\beta}_{i} \geq \hat{\beta}_{i}
$$

By a similar argument to (2.54), we obtain that $\hat{j}_{0}(s, t)>0$ for all $s, t$.

By the definition of $Q_{i+1},(2.53)$ and (2.57), we have

$$
\begin{aligned}
& y_{1}^{\left(a w_{0}(i+1)+b w_{1}(i+1)\right) d_{i+1}} \frac{P_{i+2}}{U_{i+1}^{d_{i+1}}}=\frac{Q_{i+1}}{V_{i}^{d_{i+1}}} \\
& =\left(\frac{Q_{i}^{\bar{n}_{i+1}}}{V_{i}}\right)^{d_{i+1}}+\sum_{t=0}^{d_{i+1}-1}\left(\sum_{s=1}^{\lambda_{t}} y_{1}^{a j_{0}(s, t)+b j_{1}(s, t)} \frac{Q_{0}^{\hat{j}_{0}(s, t)} Q_{1}^{j_{2}(s, t)} \cdots Q_{i-1}^{j_{i}(s, t)}}{V_{i}^{d_{i+1}-t}}\right)\left(\frac{Q_{i}^{\bar{n}_{i+1}}}{V_{i}}\right)^{t}
\end{aligned}
$$

We have

$$
\begin{aligned}
& {\left[\sum_{s=1}^{\lambda_{t}} a_{s, t} y_{1}^{a j_{0}(s, t)+b j_{1}(s, t)} \frac{Q_{0}^{\hat{j}_{0}(s, t)} Q_{1}^{j_{2}(s, t)} \cdots Q_{i-1}^{j_{i}(s, t)}}{V_{i}^{d_{i+1}-t}}\right]} \\
& \quad=\left[\sum_{s=1}^{\lambda_{t}} a_{s, t} y_{1}^{\left(a w_{0}(i+1)+b w_{1}(i+1)\right)\left(d_{i+1}-t\right)} \frac{P_{0}^{j_{0}(s, t)} P_{1}^{j_{1}(s, t)} \cdots P_{i}^{j_{i}(s, t)}}{U_{i+1}^{d_{i+1}-t}}\right] \\
& =b_{i+1, t} \alpha_{1}^{\epsilon\left(a w_{0}(i+1)+b w_{1}(i+1)\right)\left(d_{i+1}-t\right)}
\end{aligned}
$$

for $0 \leq t \leq d_{i+1}-1$ and

$$
\left[\frac{Q_{i+1}}{V_{i}^{d_{i+1}}}\right]=g_{i}\left(\hat{\alpha}_{i}\right)=0 .
$$

Thus

$$
\begin{aligned}
\hat{\beta}_{i+1} & =v\left(Q_{i+1}\right)>d_{i+1} v\left(V_{i}\right)=d_{i+1}\left(v\left(U_{i+1}\right)-w n_{1} n_{2} \cdots n_{i} \bar{n}_{i+1} \hat{\beta}_{0}\right) \\
& =n_{i+1}\left(v\left(P_{i+1}\right)-w n_{1} n_{2} \cdots n_{i} \hat{\beta}_{0}\right)=n_{i+1} \hat{\beta}_{i} .
\end{aligned}
$$

We have thus established that $A(i)$ holds for $Q_{i}$ in $R_{1}$. By induction on $i$, we have that $A(i)$ of Theorem 2.2.3 holds for $Q_{i}$ in $R_{1}$ for $1 \leq i<\Omega-1$. 
We now will show that $D(r)$ of Theorem 2.2.3 holds for the $Q_{i}$ in $R_{1}$ for all $r$. We begin by establishing the following statement:

Suppose that $\lambda \geq n_{1} w$ is as integer. Then there exist $\delta_{0}, \delta_{1} \in \mathbb{N}$ with $0 \leq \delta_{1}<\bar{n}_{1}$ such that

$$
x^{\delta_{0}+i w} y^{\delta_{1}+\left(d_{1}-1-i\right) \bar{n}_{1}}=x_{1}^{\lambda} y_{1}^{z-i \epsilon}
$$

for $0 \leq i \leq d_{1}-1$ where $z=a \delta_{0}+b\left(\delta_{1}+\left(d_{1}-1\right) \bar{n}_{1}\right.$.

We first prove (2.59). We have that

$$
(\lambda \epsilon b-r w) \bar{n}_{1}+\left(r \bar{n}_{1}-\lambda \epsilon a\right) w=\lambda
$$

for all $r \in \mathbb{Z}$. Choose $r$ so that $\delta_{1}=r \bar{n}_{1}-\lambda \epsilon a$ satisfies $0 \leq \delta_{1}<\bar{n}_{1}$. Set

$$
\delta_{0}=(\lambda \epsilon b-r w)-\left(d_{1}-1\right) w .
$$

Then

$$
(\lambda \epsilon b-r w) \bar{n}_{1}=\lambda-\delta_{1} w \geq n_{1} w-\left(\bar{n}_{1}-1\right) w=\left(n_{1}-\bar{n}_{1}+1\right) w
$$

so

$$
\delta_{0} \geq\left(n_{1}-\bar{n}_{1}-d_{1}+2\right) w=\left(\left(\bar{n}_{1}-1\right)\left(d_{1}-1\right)+1\right) w \geq w .
$$

Substituting (2.46) in $x^{\delta_{0}+i w} y^{\delta_{1}+\left(d_{1}-1-i\right) \bar{n}_{1}}$, we obtain the formula (2.59).

We now will prove that statement $D(r)$ of Theorem 2.2.3 holds for the $Q_{i}$ in $R_{1}$ for all $r$.

Suppose that we have monomials $Q_{0}^{j_{0}(l)} Q_{1}^{j_{1}(l)} \cdots Q_{r}^{j_{r}(l)}$ for $1 \leq l \leq m$ such that

$$
v\left(Q_{0}^{j_{0}(l)} Q_{1}^{j_{1}(l)} \cdots Q_{r}^{j_{r}(l)}\right)=v\left(Q_{0}^{j_{0}(1)} Q_{1}^{j_{1}(1)} \cdots Q_{r}^{j_{r}(1)}\right)
$$


for $1 \leq l \leq m$, and that we have a dependence relation in $L=V_{v} / \mathrm{m}_{v}$.

$$
0=e_{1}+e_{2}\left[\frac{Q_{0}^{j_{0}(2)} Q_{1}^{j_{1}(2)} \cdots Q_{r}^{j_{r}(2)}}{Q_{0}^{j_{0}(1)} Q_{1}^{j_{1}(1)} \cdots Q_{r}^{j_{r}(1)}}\right]+\cdots+e_{m}\left[\frac{Q_{0}^{j_{0}(m)} Q_{1}^{j_{1}(m)} \cdots Q_{r}^{j_{r}(m)}}{Q_{0}^{j_{0}(1)} Q_{1}^{j_{1}(1)} \cdots Q_{r}^{j_{r}(1)}}\right]
$$

with $e_{i} \in \mathfrak{F}\left(\alpha_{1}\right)$ (and some $e_{i} \neq 0$ ). Multiplying the $Q_{0}^{j_{0}(l)} Q_{1}^{j_{1}(l)} \cdots Q_{r}^{j_{r}(l)}$ for $1 \leq l \leq m$ by a common term $Q_{0}^{t}$ with $t$ a sufficiently large positive integer, we may assume that

$$
\hat{j}_{0}(l)=j_{0}(l)-j_{1}(l) w n_{1}-j_{2}(l) w n_{1} n_{2}-\cdots-j_{r}(l) w n_{1} n_{2} \cdots n_{r} \geq n_{1} w
$$

for $1 \leq l \leq m$. We have that

$$
Q_{0}^{j_{0}(l)} Q_{1}^{j_{1}(l)} \cdots Q_{r}^{j_{r}(l)}=Q_{0}^{\hat{j}_{0}(l)} P_{2}^{j_{1}(l)} \cdots P_{r+1}^{j_{r}(l)}
$$

Since $\hat{j}_{0}(l) \geq w n_{1},(2.59)$ implies that for each $l$ with $1 \leq l \leq w$, there exist $\delta_{0}(l), \delta_{1}(l)$ with $\delta_{0}(l), \delta_{1}(l) \in \mathbb{N}$ and $0 \leq \delta_{1}(l)<\bar{n}_{1}$ such that

$$
P_{0}^{\delta_{0}(l)+i w} P_{1}^{\delta_{1}(l)+\left(d_{1}-1-i\right) \bar{n}_{1}}=y_{1}^{z(l)-i \epsilon} Q_{0}^{\hat{j}_{0}(l)}
$$

for $0 \leq i \leq d_{1}-1$. The ordered set

$$
\left\{\alpha_{1}^{\epsilon(z(l)-z(1))}, \alpha_{1}^{\epsilon(z(l)-z(1))-1}, \cdots, \alpha_{1}^{\epsilon(z(l)-z(1))-\left(d_{1}-1\right)}\right\}
$$

is a $\mathfrak{f}$-basis of $\mathfrak{f}\left(\alpha_{1}\right)$ for all $l$ (since multiplication by $\alpha_{1}^{\epsilon(z(l)-z(1))+\left(d_{1}-1\right)}$ is a $\mathfrak{k}$-vector space isomorphism of $\mathfrak{f}\left(\alpha_{1}\right)$, and thus takes a basis to a basis). Thus there exists $e_{l, i} \in \mathfrak{f}$ such that

$$
e_{l}=\sum_{i=0}^{d_{1}-1} e_{l, i} \alpha_{1}^{\epsilon(z(l)-z(1))-i}
$$

Since some $e_{l, i} \neq 0$, we have a dependence relation

$$
0=\sum_{l=1}^{m} \sum_{i=0}^{d_{1}-1} e_{l, i}\left[\frac{P_{0}^{\delta_{0}(l)+i w} P_{1}^{\delta_{1}(l)+\left(d_{1}-1-i\right) \bar{n}_{1}} P_{2}^{j_{1}(l)} \cdots P_{r+1}^{j_{r}(l)}}{P_{0}^{\delta_{0}(1)} P_{1}^{\delta_{1}(1)+\left(d_{1}-1\right) \bar{n}_{1}} P_{2}^{j_{1}(1)} \cdots P_{r+1}^{j_{r}(1)}}\right],
$$

a contradiction to $D(r+1)$ of Theorem 2.2.3 for the $P_{i}$ in $R$. Thus we have established $D(r)$ of Theorem 2.2.3 for the $Q_{i}$ in $R_{1}$. 


\subsection{Polynomial Rings in two Variables}

The algorithm of Theorem 2.2.3 is applicable when $R=\mathfrak{f}[x, y]$ is a polynomial ring over a field and $v$ is a valuation which dominates the maximal ideal $(x, y)$ of $R$. In this case the calculations become much simpler, as we now indicate (of course we take the coefficient set $C S$ to be the field $\mathfrak{f}$ ). In the case when $R$ is equicharacteristic, we can establish from the polynomial case the results of this paper using Cohen's structure theorem and Proposition 2.1.4 to reduce to the case of a polynomial ring in two variables.

If $f \in R=\mathfrak{f}[x, y]$ is a nonzero polynomial, then we have an expansion $f=$ $a_{0}(x)+a_{1}(x) y+\cdots+a_{r}(x) y^{r}$ where $a_{i}(x) \in \mathfrak{f}[x]$ for all $i$ and $a_{r}(x) \neq 0$. We define $\operatorname{ord}_{y}(f)=r$, and say that $f$ is monic in $y$ if $a_{r}(x) \in \mathfrak{f}$. We first establish the following formula.

$$
P_{i} \text { is monic in } y \text { with } \operatorname{deg}_{y} P_{i}=n_{1} n_{2} \cdots n_{i-1} \text { for } i \geq 2 \text {. }
$$

We establish (2.60) by induction. In the expansion (2.10) of $P_{i+1}$, we have for $0 \leq t \leq d_{i}-1$ and whenever $a_{s, t} \neq 0$, that $0 \leq j_{k}(s, t)<n_{k}$ for $1 \leq k \leq i-1$. Thus

$$
\begin{aligned}
& \operatorname{deg}_{y}\left(P_{0}^{j_{0}(s, t)} P_{1}^{j_{1}(s, t)} \cdots P_{i-1}^{j_{i-1}(s, t)} P_{i}^{t \bar{n}_{i}}\right) \\
& \quad=j_{1}(s, t)+j_{2}(s, t) n_{1}+j_{3}(s, t) n_{1} n_{2}+\cdots+j_{i-1} n_{1} n_{2} \cdots n_{i-2}+t \bar{n}_{i} n_{1} n_{2} \cdots n_{i-1} \\
& \quad<n_{1} n_{2} \cdots n_{i} .
\end{aligned}
$$

Thus $\operatorname{deg}_{y} P_{i+1}=\operatorname{deg}_{y} P_{i}^{n_{i}}=n_{1} n_{2} \cdots n_{i}$. We further see that $P_{i+1}$ is monic in $y$.

Set $\sigma(0)=0$ and for $i \geq 1$ let

$$
\sigma(i)=\min \left\{j \mid j>\sigma(i-1) \text { and } n_{j}>1\right\} .
$$

Let $Q_{i}=P_{\sigma(i)}$. We calculate (as long as we are not in the case $\Omega=\infty$ and $n_{i}=1$ for $i \gg 0$ ) that for $d \in \mathbb{Z}_{+}$, there exists a unique $r \in \mathbb{Z}_{+}$and $j_{1}, \ldots, j_{r} \in \mathbb{Z}_{+}$such 
that $0 \leq j_{k}<n_{k}$ for $1 \leq k \leq r$ and $\operatorname{deg}_{y} Q_{1}^{j_{1}} \cdots Q_{r}^{j_{r}}=d$. Let $M_{d}$ be this monomial. Since the monomials $M_{d}$ are monic in $y$, we see (continuing to assume that we are not in the case $\Omega=\infty$ and $n_{i}=1$ for $i \gg 0$ ) that if $f \in R=\mathfrak{f}[x, y]$ is nonzero with $\operatorname{deg}_{y}(f)=d$, then there is a unique expression

$$
f=\sum_{i=0}^{d} A_{i}(x) M_{i}
$$

where $A_{i}(x) \in \mathfrak{f}[x]$, and

$$
v(f)=\min _{i}\left\{\operatorname{ord}\left(A_{i}\right) v\left(Q_{0}\right)+v\left(M_{i}\right)\right\}
$$

In the case when $\Omega=\infty$ and $n_{i}=1$ for $i \gg 0$ we have a similar statement, but we may need to introduce a new polynomial $g$ of "infinite value" as in Case 3 of Theorem 2.2.13.

\subsection{The $A_{2}$ Singularity}

Lemma 2.7.1 Let $\mathfrak{f}$ be an algebraically closed field, and let $A=\mathfrak{f}\left[x^{2}, x y, y^{2}\right]$, a subring of the polynomial ring $B=\mathfrak{f}[x, y]$. Let $\mathrm{m}=\left(x^{2}, x y, y^{2}\right) A$ and $\mathfrak{n}=(x, y) B$. Suppose that $v$ is a rational nondiscrete valuation dominating $B_{n}$, such that $v$ has a generating sequence

$$
P_{0}=x, P_{1}=y, P_{2}, \ldots
$$

in $\mathrm{E}[x, y]$ of the form of the conclusions of Theorem 2.2.3, such that each $P_{i}$ is a $\mathrm{f}$-linear combinations of monomials in $x$ and $y$ of odd degree, and

$$
\beta_{0}=v(x), \beta_{1}=v(y), \beta_{2}=v\left(P_{2}\right), \ldots
$$

is the increasing sequence of minimal generators of $S^{B_{n}}(v)$, with $\beta_{i+1}>\bar{n}_{i} \beta_{i}$ for $i \geq 1$, where

$$
\bar{n}_{i}=\left[G\left(\beta_{0}, \ldots, \beta_{i}\right): G\left(\beta_{0}, \ldots, \beta_{i-1}\right)\right] .
$$


Then

$$
S^{A_{\mathrm{m}}}(v)=\left\{\begin{array}{l}
a_{0} \beta_{0}+a_{1} \beta_{1}+\cdots+a_{i} \beta_{i} \mid i \in \mathbb{N}, a_{0}, \ldots, a_{i} \in \mathbb{N} \\
\text { and } a_{0}+a_{1} \cdots+a_{i} \equiv 0 \bmod 2
\end{array}\right\}
$$

Proof For $f \in \mathfrak{f}[x, y]$, let $t=\operatorname{deg}_{y}(f)$. By (2.61), $f$ has a unique expansion

$$
f=\sum_{i=0}^{t}\left(\sum_{k} b_{k, i} x^{k}\right) P_{1}^{j_{1}(i)} \cdots P_{r}^{j_{r}(i)}
$$

where $b_{k, i} \in \mathfrak{F}, 0 \leq j_{k}(i)<\bar{n}_{k}$ for $1 \leq k$ and

$$
\operatorname{deg}_{y} P_{1}^{j_{1}(i)} \cdots P_{r}^{j_{r}(i)}=i
$$

for all $i$. Looking first at the $t=\operatorname{deg}_{y}(f)$ term, and then at lower order terms, we see that $f \in \mathfrak{f}\left[x^{2}, x y, y^{2}\right]$ if and only if $k+j_{1}(i)+\cdots+j_{r}(i) \equiv 0 \bmod 2$ whenever $b_{k, i} \neq 0$.

Example 2.7.2 Suppose that $\mathfrak{f}$ is a field and $R$ is the localization of $\mathfrak{E}[u, v, w] / u v-w^{2}$ at the maximal ideal $(u, v, w)$. Then there exists a rational nondiscrete valuation $v$ dominating $R$ such that if

$$
\gamma_{0}<\gamma_{1}<\cdots
$$

is the increasing sequence of minimal generators of the semigroup $S^{R}(v)$, then given $n \in \mathbb{Z}_{+}$, there exists $i>n$ such that $\gamma_{i+1}=\gamma_{i}+\frac{\gamma_{0}}{3}$ and $\gamma_{i+1}$ is in the group generated by $\gamma_{0}, \ldots, \gamma_{i}$

Proof Let $A=\mathfrak{f}[x, y]$ be a polynomial ring with maximal ideal $\mathfrak{n}=(x, y) \mathfrak{f}[x, y]$. We will use the criterion of Theorem 2.4.1 to construct a rational nondiscrete valuation $v$ dominating $T=A_{\mathfrak{n}}$, with a generating sequence

$$
P_{0}=x, P_{0}=y, P_{2}, \ldots
$$


such that

$$
\beta_{0}=v(x), \beta_{1}=v(y), \beta_{2}=v\left(P_{2}\right), \ldots
$$

is the increasing set of minimal generators of the semigroup $S^{T}(v)$. We will construct the $P_{i}$ so that each $P_{i}$ is a $\mathfrak{f}$-linear combination of monomials in $x$ and $y$ of odd degree.

We define the first part of a generating sequence by setting

$$
P_{0}=x, P_{1}=y, P_{2}=y^{3}-x^{5},
$$

with $\beta_{0}=v(x)=1, \beta_{1}=v(y)=\frac{5}{3}$. Set $b_{1}=0$.

We now inductively define

$$
P_{i+1}=P_{i}^{3}-x^{a_{i}} P_{i-1}
$$

with $a_{i}$ an even positive integer, and $\beta_{i}=v\left(P_{i}\right)=b_{i}+\frac{5}{3^{i}}$ with $b_{i} \in \mathbb{Z}_{+}$, for $i \geq 2$, by requiring that 3 divides $a_{i}+b_{i-1}$ and

$$
b_{i}=\frac{a_{i}+b_{i-1}}{3}>3 b_{i-1}+5
$$

for $i \geq 2$. $a_{i}, b_{i}$ satisfying these relations can be constructed inductively from $b_{i-1}$.

Now let $B=\mathfrak{f}\left[x^{2}, x y, y^{2}\right], \mathfrak{m}=\left(x^{2}, x y, y^{2}\right) B$, so that $R \cong B_{\mathfrak{m}}$. With this identification, the semigroup $S^{R}(v)$ is generated by $\left\{\beta_{i}+\beta_{j} \mid i, j \in \mathbb{N}\right\}$. From $3 \beta_{i}<\beta_{i+1}$ for $i \geq 1$ and $\beta_{i}<\beta_{j}$ if $j>i$, we conclude that if $i \leq j, k \leq l$ and $j<l$, then

$$
\beta_{i}+\beta_{j}<\beta_{k}+\beta_{l}
$$

Let

$$
\gamma_{0}=2<\gamma_{1}<\cdots
$$


be the sequence of minimal generators of the semigroup $S^{R}(v)$. By (2.63), for $n \in \mathbb{Z}_{+}$, there exists an index $l$ such that $\gamma_{l}=\beta_{0}+\beta_{n}$. We have $l \geq n$. The semigroup $S\left(\gamma_{0}, \gamma_{1}, \ldots, \gamma_{l}\right)$ is generated by

$$
\left\{\beta_{i}+\beta_{j} \mid i \leq j \text { and } j \leq n-1\right\}
$$

and $\beta_{0}+\beta_{n}$.

Suppose $\beta_{1}+\beta_{n} \in S\left(\gamma_{0}, \gamma_{1}, \ldots, \gamma_{l}\right)$. Since $S\left(\gamma_{0}, \ldots, \gamma_{l-1}\right) \subset \frac{1}{3^{n-1}} \mathbb{Z}$, we have an expression $\beta_{1}+\beta_{n}=r \gamma_{l}+\tau$ with $r$ a positive integer, and $\tau \in S\left(\gamma_{0}, \ldots, \gamma_{l-1}\right)$. Now

$$
\gamma_{l}=\beta_{0}+\beta_{n}=1+b_{n}+\frac{5}{3^{n}}
$$

and

$$
\beta_{1}+\beta_{n}=\frac{5}{3}+b_{n}+\frac{5}{3^{n}}
$$

implies $\tau \leq \frac{5}{3}-1=\frac{2}{3}$, which is impossible, since $\gamma_{0}=\beta_{0}+\beta_{0}=2$. Thus $\beta_{1}+\beta_{n} \notin$ $S\left(\gamma_{0}, \gamma_{1}, \ldots, \gamma_{l}\right)$ and $\beta_{1}+\beta_{n}=\gamma_{l+1}$ is the next largest minimal generator of $S^{R}(v)$.

We have that $\gamma_{l+1}=\beta_{1}+\beta_{n}=\left(\beta_{0}+\beta_{1}\right)+\left(\beta_{0}+\beta_{n}\right)-2 \beta_{0}$ is in the group generated by $\gamma_{0}, \ldots, \gamma_{l}$.

Example 2.7.3 Let notation be as in Example 2.7.2 and its proof. Then $R \rightarrow T$ is finite, but $S^{T}(v)$ is not a finitely generated $S^{R}(v)$ module.

Proof Suppose $S^{T}(v)$ is a finitely generated $S^{R}(v)$ module. Then there exists $n>0$ such that $S^{T}(v)$ is generated by $\beta_{0}, \ldots, \beta_{n}$ and $\left\{\beta_{i}+\beta_{j} \mid i, j \in \mathbb{N}\right\}$. For $l>n, \beta_{l}$ cannot be in this semigroup.

Example 2.7.4 Let $A=\mathfrak{f}[u, v]_{(u, v)}$. Then $A \rightarrow T$ is a finite extension of regular local rings, but $S^{T}(v)$ is not a finitely generated $S^{A}(v)$ module. 
Proof Since $A$ is a subring of $R, S^{A}(v)$ is a subsemigroup of $S^{R}(v)$. Since $S^{T}(v)$ is not a finitely generated $S^{R}(v)$-module, by Example 2.7.3, $S^{T}(v)$ cannot be a finitely generated $S^{A}(v)$-module. 


\section{CHAPTER 3}

\section{RAMIFICATION OF LOCAL RINGS ALONG VALUATIONS}

In this chapter we discuss stable forms of extensions of algebraic local rings along a valuation in all dimensions over a field $\mathfrak{f}$ of characteristic zero, and generalize a formula of Ghezzi, Ha and Kashcheyeva describing the extension of associated graded rings along the valuation for stable extensions of regular algebraic local rings of dimension two to arbitrary ground fields $\npreceq$ of characteristic zero. We discuss the failure of this result in positive characteristic. The results in this chapter have appeared in [21].

\subsection{Monomial Transforms and Stable Extensions}

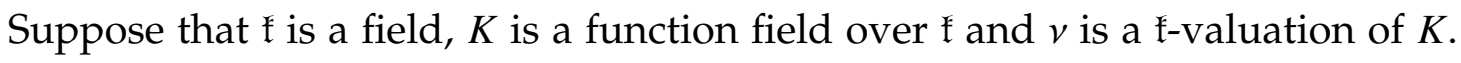
A local ring $R$ is said to be an algebraic local ring of $K$ if it is essentially of finite type over $\mathfrak{f}$ and its quotient field is $K$. A monoidal transform $R \rightarrow R_{1}$ of $R$ is a local ring $R_{1}$ of the blowup of a regular prime ideal $\mathfrak{p}$ of $R\left(R / \mathfrak{p}\right.$ is regular). $R \rightarrow R_{1}$ is a quadratic transform if $R_{1}$ is a local ring of the blow up of the maximal ideal of $R . R \rightarrow R_{1}$ is a monoidal transform along $v$ if $V_{v}$ dominates $R_{1}$.

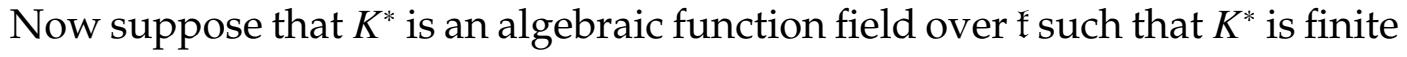
separable over $K$, and $v^{*}$ is a valuation of $K^{*}$ which is an extension of $v$. Let

$$
n=\operatorname{trdeg}_{\mathfrak{t}} K^{*}-\operatorname{trdeg} V_{v^{*}} / m_{v^{*}} .
$$

Let

$$
e=\left[\Gamma_{v^{*}}: \Gamma_{v}\right] \text { and } f=\left[V_{v^{*}} / m_{v^{*}}: V_{v} / m_{v}\right]
$$


be the reduced ramification index and relative degree of $v^{*}$ over $v$.

Suppose that $R$ and $S$ are algebraic local rings for $K$ and $K^{*}$ such that $S$ dominates $R$ and $v^{*}$ dominates $S$ (so that $v$ dominates $R$ ). We will say that $R \rightarrow S$ is monomial if $R$ and $S$ are $n$-dimensional regular local rings and there exist regular parameters $x_{1}, \ldots, x_{n}$ in $R, y_{1}, \ldots, y_{n}$ in $S$, an $n \times n$ matrix $A=\left(a_{i j}\right)$ of natural numbers with $\operatorname{det}(A) \neq 0$ and units $\delta_{i} \in S$ such that

$$
x_{i}=\delta_{i} \prod_{j=1}^{n} y_{j}^{a_{i j}}
$$

for $1 \leq i \leq n$. In Theorem 1.1 [13] it is proven that when the ground field $k$ has characteristic zero, there exists a commutative diagram

$$
\begin{array}{ccc}
R_{0} & \rightarrow & S_{0} \\
\uparrow & & \uparrow \\
R & & \rightarrow
\end{array}
$$

such that the vertical arrows are products of monoidal transforms along $v^{*}$ and $R_{0} \rightarrow S_{0}$ is monomial. It is shown in Theorem 5.1 [13] and Theorem 4.8 [18] that the matrix $A_{0}$ describing $R_{0} \rightarrow S_{0}$ (with respect to regular parameters $x_{1}(0), \ldots, x_{n}(0)$ in $R_{0}$ and $y_{1}(0), \ldots, y_{n}(0)$ in $\left.S_{0}\right)$ can be required to take a very special block form, which reflects the rank and rational rank of $v^{*}$. We will say that $R_{0} \rightarrow S_{0}$ is strongly monomial if it is monomial and the matrix $A_{0}$ has this special block form.

In Theorem 6.1 [18] it is shown (assuming that $£$ has characteristic zero) that we can always find a diagram (3.2) such that the following conditions hold:

1) $R_{0} \rightarrow S_{0}$ is strongly monomial. 
2) If

$$
\begin{array}{ccc}
R_{1} & \rightarrow & S_{1} \\
\uparrow & & \uparrow \\
R_{0} & \rightarrow & S_{0}
\end{array}
$$

is such that $R_{1} \rightarrow S_{1}$ is strongly monomial with respect to regular parameters $x_{1}(1), \ldots, x_{n}(1)$ in $R_{1}$ and $y_{1}(1), \ldots, y_{n}(1)$ in $S_{1}$, and the vertical arrows are products of monoidal transforms, then

2a) The natural group homomorphism

$$
\mathbb{Z}^{n} / A_{1}^{t} \mathbb{Z}^{n} \rightarrow \Gamma_{v^{*}} / \Gamma_{v}
$$

defined by

$$
\left(b_{1}, \ldots, b_{n}\right) \mapsto\left[b_{1} v^{*}\left(y_{1}(1)\right)+\cdots+b_{n} v^{*}\left(y_{n}(1)\right)\right]
$$

is an isomorphism (where $A_{1}$ is the matrix of exponents of $R_{1} \rightarrow S_{1}$ with respect to our given systems of parameters).

2b) $V_{v^{*}} / m_{v^{*}}$ is the join $V_{v^{*}} / m_{v^{*}}=\left(V_{v} / m_{v}\right)\left(S_{1} / m_{S_{1}}\right)$.

2c) $V_{v} / m_{v}$ and $S_{1} / m_{S_{1}}$ are linearly disjoint over $R_{1} / m_{R_{1}}$ in $V_{v^{*}} / m_{v^{*}}$.

Lemma 3.1.1 Suppose that $V_{v^{*}} / m_{v^{*}}=\left(V_{v} / m_{v}\right)\left(S / m_{v}\right)$. Then $\left[S / m_{S}: R / m_{R}\right]=f$ if and only if $V_{v} / m_{v}$ and $S / m_{S}$ are linearly disjoint in $V_{v^{*}} / m_{v^{*}}$ over $R / m_{R}$.

Proof Suppose that $\left[S / m_{S}: R / m_{R}\right]=f$. Let $h_{1}, \ldots, h_{S} \in S / m_{S}$ be linearly independent over $R / m_{R}$. Extend this set to a basis $h_{1}, \ldots, h_{f}$ of $S / m_{S}$ over $R / m_{R}$. Then $h_{1}, \ldots, h_{f}$ span $V_{v^{*}} / m_{v^{*}}$ over $V_{v} / m_{v}$, so they are linearly independent over $V_{v} / m_{v}$.

Now suppose that $V_{v} / m_{v}$ and $S / m_{S}$ are linearly disjoint over $R / m_{R}$. There exist $\alpha_{1}, \ldots, \alpha_{f} \in S / m_{S}$ which are a basis of $V_{v^{*}} / m_{v^{*}}$ over $V_{v} / m_{v}$. Then $\alpha_{1}, \ldots, \alpha_{f}$ 
are linearly independent over $R / m_{R}$, so $\left[S / m_{S}: R / m_{R}\right] \geq f$. However, a basis of $S / m_{S}$ over $R / m_{R}$ is linearly independent over $V_{v} / m_{v}$, so $\left[S / m_{S}: R / m_{R}\right]=f$.

Theorem 6.1 [18] and Lemma 3.1.1 implies that, given $R \rightarrow S$, there exists a monomial extension $R_{0} \rightarrow S_{0}$ as in (3.2) satisfying 1) and 2) above. In Theorem 6.3 [18] it is shown that the extension $V \rightarrow V^{*}$ can naturally be understood as

a direct limit of $R_{0} \rightarrow S_{0}$ as above. We will say that $R_{0} \rightarrow S_{0}$ is stable if the conclusions 1) and 2) above hold.

If $R \rightarrow S$ is stable, we have that

$$
e=\operatorname{det}(A) \text { and } f=\left[S / m_{S}: R / m_{R}\right] \text {. }
$$

where $e$ is the reduced ramification index and $f$ is the relative degree of $v^{*}$ over $v$.

\subsection{Stable Extensions along Abhyankar Valuations}

Suppose that is a field, $K^{*} / K$ is an algebraic extension of algebraic function fields over $\mathfrak{f}, v$ is a $\mathfrak{f}$-valuation of $K$ and $v^{*}$ is an extension of $v$ to $K^{*}$. Suppose that $R$ and $S$ are algebraic local ring of $K$ and $K^{*}$ such that $v^{*}$ dominates $S$ and $S$ dominates $R$. We recall that Abhyankar valuations are valuations that satisfy the equality in Abhyankar's inequality (1.1).

Proposition 3.2.1 Suppose that $£$ has characteristic zero, $v$ is an Abhyankar valuation and $R \rightarrow S$ is stable. Then we have a natural isomorphism of graded rings

$$
g r_{v^{*}}(S) \cong\left(g r_{v}(R) \otimes_{R / m_{R}} S / m_{S}\right)\left[\bar{y}_{1}, \ldots, \bar{y}_{n}\right]
$$


where $\bar{y}_{1}, \ldots, \bar{y}_{n}$ are the initial forms of $y_{1}, \ldots, y_{n}$, with the only relations being

$$
\left[x_{i}\right]=\left[\delta_{i}\right] \bar{y}_{1}^{a_{i 1}} \cdots \bar{y}_{n}^{a_{i n}} \quad 1 \leq i \leq n
$$

obtained from (3.1) ([ $\left[\delta_{i}\right]$ is the class of $\delta_{i}$ in $\left.S / m_{S}\right)$. The degree of the extension of quotient fields of

$$
g r_{v}(R) \rightarrow g r_{v^{*}}(S)
$$

is ef.

Proof Since $R \rightarrow S$ is stable, and $v^{*}$ and $v$ are Abhyankar valuations, we have that $v^{*}\left(y_{1}\right), \ldots, v^{*}\left(y_{n}\right)$ is a $\mathbb{Z}$-basis of $\Gamma_{v^{*}}$ and $v\left(x_{1}\right), \ldots, v\left(x_{n}\right)$ is a $\mathbb{Z}$-basis of $\Gamma_{v}$.

By Hensel's lemma, $\hat{R} \cong \mathfrak{f}^{\prime}\left[\left[x_{1}, \ldots, x_{n}\right]\right]$ where $\mathfrak{f}^{\prime} \cong R / m_{R}$ is a coefficient field of $\hat{R}$. Since $v\left(x_{1}\right), \ldots, v\left(x_{n}\right)$ are rationally independent, $v$ has a unique extension to a valuation $\hat{v}$ of the quotient field of $\hat{R}$, defined by

$$
\hat{v}(f)=\min \left\{i_{1} v\left(x_{1}\right)+\cdots+i_{n} v\left(x_{n}\right) \mid a_{i_{1}, \ldots, i_{n}} \neq 0\right\}
$$

if $f=\sum a_{i_{1}, \ldots, i_{n}} x_{1}^{i_{1}} \cdots x_{n}^{i_{n}} \in \mathfrak{f}^{\prime}\left[\left[x_{1}, \ldots, x_{n}\right]\right]$ (with $a_{i_{1}, \ldots, i_{n}} \in \mathfrak{f}^{\prime}$ ). Since distinct monomials have distinct values, we have an isomorphism of residue fields $V_{v} / m_{v} \cong R / m_{R}$.

Hence $\operatorname{gr}_{v}(R) \cong R / m_{R}\left[\bar{x}_{1}, \ldots, \bar{x}_{n}\right]$, is a polynomial ring, where $\bar{x}_{i}$ is the class of $x_{i}$, with the grading $\operatorname{deg} \bar{x}_{i}=v\left(x_{i}\right)$. Further $\operatorname{gr}_{v^{*}}(S) \cong S / m_{S}\left[\bar{y}_{1}, \ldots, \bar{y}_{n}\right]$, is a polynomial ring, where $\bar{y}_{i}$ is the class of $y_{i}$. The proposition follows. 


\subsection{Stable Extensions along non-Abhyankar Valuations in Di- mension Two}

In this section, we adopt the situation of $\mathfrak{x}, K^{*} / K, v^{*} / v$ and $R \rightarrow S$ from that of Section §3.2. If $v$ is an Abhyankar valuation, and $R \rightarrow S$ is quasi-finite, we have that $S^{S}\left(v^{*}\right)$ is finitely generated as a module over the semigroup $S^{R}(v)$ by Proposition 3.2.1.

It is natural to ask if an analog of Proposition 3.2.1 holds for more general valuations. We have the essential difference that the valuation groups $\Gamma_{v}$ are not finitely generated in general. There even exist examples where $R \rightarrow S$ is quasifinite but $S^{S}\left(v^{*}\right)$ is not a finitely generated module over $S^{R}(v)$. In Section $\S 2.7$, an example is given of a finite monomial extension of two dimensional regular algebraic local rings (over any ground field) such that $S^{S}\left(v^{*}\right)$ is not a finitely generated module over $S^{R}(v)$. This example is necessarily not stable. Some other examples are given in [19] showing bad behavior of $S^{S}\left(v^{*}\right)$ over $S^{R}(v)$.

However, the conclusions of Proposition 3.2.1 always hold for stable mappings $R \rightarrow S$ when $R$ and $S$ have dimension two $(n=2)$. By Abhyankar's inequality (1.1), when $n=2, v$ is an Abhyankar valuation unless $v$ is rational and 0-dimensional, i.e. rat.rank $v=1$ and $\operatorname{tr} \cdot \operatorname{deg}_{\mathrm{f}} V_{v} / \mathfrak{m}_{v}=0$. We have the following theorem, which generalizes Proposition 3.2.1 to this case. This surprising theorem was proven when $k$ is algebraically closed of characteristic zero and $\operatorname{dim} K=2$ by Ghezzi, Ha and Kashcheyeva in [24]. If $n=2, v$ is rational and $R \rightarrow S$ is stable, then $R$ has regular parameters $u, v, S$ has regular parameters $x, y$ and there exist a unit $\gamma$ in $S$ such that

$$
u=\gamma x^{e}, v=y,
$$


where $e=\left|\Gamma_{v^{*}} / \Gamma_{v}\right|$ is the reduced ramification index.

Theorem 3.3.1 Suppose that $\mathfrak{f}$ is a field of characteristic zero, $v^{*}$ is a rational 0 dimensional valuation, $n=2$ and $R \rightarrow S$ is stable. Then

$$
\operatorname{gr}_{v^{*}}(S) \cong\left(\operatorname{gr}_{v}(R) \otimes_{R / m_{R}} S / m_{S}\right)[Z] /\left(Z^{e}-\left[\gamma_{0}\right]^{-1}[u]\right),
$$

and the degree of the extension of quotient fields of $g r_{v}(R) \rightarrow g r_{v^{*}}(S)$ is ef.

The remaining sections of this chapter are devoted to the proof of this theorem. Our proof requires the construction of generating sequences for valuations in arbitrary regular local rings of dimension two in [22]. Theorem 3.3.1 is proven in Section §3.6, as a consequence of Proposition 3.6.1, which shows that a generating sequence in $R$ is almost a generating sequence in $S$ if $R \rightarrow S$ is stable.

In contrast to the fact that finite generation may not hold even for a monomial mapping, when $v^{*}$ is a rational 0-dimensional valuation with $n=2$ (Example 9.4 [22]), we have finite generation if $R \rightarrow S$ is stable.

Corollary 3.3.2 Suppose that $\mathfrak{f}$ is a field of characteristic zero, $v^{*}$ is a rational 0 dimensional valuation, $n=2$ and $R \rightarrow S$ is stable. Then the semigroup $S^{S}\left(v^{*}\right)$ is a finitely generated $S^{R}(v)$-module.

An interesting question is if an analogue of the conclusions of Proposition 3.2.1 holds in general for any $n$ and arbitrary valuations for stable mappings over fields $k$ of characteristic zero. It would be remarkable if this were true.

With some small modification in the definition of strongly monomial (in (3.2)), strong monomialization holds for Ahhyankar valuations in positive characteristic, as follows from [32], (a strong form of local uniformization is proven 
for Abhyankar valuations by Knaf and Kuhlmann), and thus Proposition 3.2.1 holds in positive characteristic. A description of $\operatorname{gr}_{v}(R)$ for $v$ an Abhyankar valuation dominating a (singular) local ring $R$, over an algebraically closed field of arbitrary characteristic, and a proof of local uniformization for Abhyankar valuations derived from this construction, has been recently given by Teissier in [44].

Over fields of positive characteristic, it is shown in Section 7.11 of [18] that the strong monomialization theorem is not true, even when $n=2$, $\mathrm{f}$ is algebraically closed and $v$ is rational and zero dimensional. It is not known if monomialization holds, although it seems unlikely. Stable forms are given in [18] for mappings in dimension two over an algebraically closed field of positive characteristic which are much more complicated than in the characteristic zero case. The fundamental obstruction to obtaining strong monomialization is the defect. It is shown in [18] that strong monomialization holds in dimension two over algebraically closed fields $\mathfrak{f}$ for extensions of valuations for which there is no defect. In [25], Ghezzi and Kashcheyeva prove Theorem 3.3.1 when $\mathfrak{f}$ is algebraically closed of positive characteristic, $\operatorname{dim} K=2$ and the extension has no defect.

In the example of Section 7.11 of [18], the stable forms $R_{i} \rightarrow S_{i}$ satisfy

$$
\operatorname{gr}_{v}\left(R_{i}\right) \rightarrow \mathrm{gr}_{v^{*}}\left(S_{i}\right)
$$

is integral but not finite, in contrast to the case of Proposition 3.2.1 and Theorem 3.3.1. In fact, $\operatorname{gr}_{v}\left(R_{i}\right)=\operatorname{gr}_{v^{*}}\left(S_{i}\right)^{p}$. Further, $S^{S_{i}}\left(v^{*}\right)$ is not a finitely generated $S^{R_{i}}(v)$ module for any $i$. In this example, the degree of the extension of quotient fields of (3.4) is $e f p^{\delta\left(v^{*} / v\right)}=p^{2}$, where $\delta\left(v^{*} / v\right)=2$ is the defect of $v^{*}$ over $v$. The defect is always zero in characteristic zero, and for Abhyankar valuations. 


\subsection{A modification of the algorithm}

Suppose that $f$ is a field of characteristic zero and $K$ is a 2-dimensional algebraic function field over $\mathrm{f}$. Suppose that $v$ is a rational 0 -dimensional valuation of $K$. Suppose that $R$ is a regular algebraic local ring of $K$ such that $v$ dominates $R$.

Let

$$
R \rightarrow T_{1} \rightarrow T_{2} \rightarrow \cdots
$$

be the sequence of quadratic transforms of $R$ along $v$, so that $V_{v}=\cup T_{i}$. Suppose that $x, y$ are regular parameters in $R$. There exists a smallest value $i$ such that the divisor of $x y$ in $\operatorname{spec}\left(T_{i}\right)$ has only one component.

$$
\text { Define } R_{1}=T_{i} \text {. }
$$

We consider the algorithm of Theorem 2.2.3 to construct a generating sequence in $R$ with Remark 2.2.4 and the following observation: We can replace $U_{i}$ with a unit $\tau_{i} \in R$ times $U_{i}$ in the algorithm. The algorithm (which we will call the modified algorithm to construct a generating sequence) iterates in the following way. Suppose that for $i \geq 0$ we have constructed the first $i+1$ terms

$$
P_{0}=x, P_{1}=y, P_{2}, \ldots, P_{i}
$$

of a generating sequence by the (modified) algorithm. To produce the next term $P_{i+1}$, the algorithm proceeds as follows. First we compute

$$
\bar{n}_{i}=\left[G\left(v\left(P_{0}\right), \ldots, v\left(P_{i}\right)\right): G\left(v\left(P_{0}\right), \ldots, v\left(P_{i-1}\right)\right)\right] .
$$

This allows us to find a suitable element

$$
U_{i}=P_{0}^{\omega_{0}(i)} P_{1}^{\omega_{1}(i)} \cdots P_{i-1}^{\omega_{i-1}(i)} \tau_{i}
$$


with $\tau_{i} \in R$ an arbitary unit, such that $v\left(P_{i}^{\bar{n}_{i}}\right)=v\left(U_{i}\right)$. Let

$$
\alpha_{i}=\left[\frac{P_{i}^{\bar{n}_{i}}}{U_{i}}\right] \in V_{v} / m_{v}
$$

and

$$
f_{i}(z)=z^{d_{i}}+b_{i, d_{i}-1} z^{d_{i}-1}+\cdots+b_{i, 0}
$$

be the minimal polynomial of $\alpha_{i}$ over $R / m_{R}\left(\alpha_{1}, \ldots, \alpha_{i-1}\right)$. Then the algorithm produces an element $P_{i+1} \in R$ of the form

$$
P_{i+1}=P_{i}^{\bar{n}_{i} d_{i}}+\sum_{t=0}^{d_{i}-1}\left(\sum_{s=1}^{\lambda_{t}} a_{s, t} P_{0}^{j_{0}(s, t)} \cdots P_{i-1}^{j_{i-1}(s, t)}\right) P_{i}^{t \bar{n}_{i}}
$$

where $a_{s, t} \in R$ are units, $j_{0}(s, t), \ldots, j_{i-1}(s, t) \in \mathbb{N}$ with $0 \leq j_{k}(s, t)<n_{k}$ for $k \geq 1$ and $0 \leq t<d_{i}$ such that

$$
v\left(P_{0}^{j_{0}(s, t)} \cdots P_{i-1}^{j_{i-1}(s, t)} P_{i}^{t \bar{n}_{i}}\right)=\bar{n}_{i} d_{i} v\left(P_{i}\right)
$$

for all $s, t$, and

$$
b_{i, t}=\left[\sum_{s=1}^{\lambda_{t}} a_{s, t} \frac{P_{0}^{j_{0}(s, t)} \cdots P_{i-1}^{j_{i-1}(s, t)}}{U_{i}^{d_{i}-t}}\right] \in V_{v} / m_{v} .
$$

Then $P_{0}, P_{1}, \ldots, P_{i}, P_{i+1}$ are the first $i+2$ terms of a generating sequence for $v$ in $R$.

The observation of Remark 2.2.4 is that any choice of (3.8) such that (3.9) holds gives an extension $P_{i+1}$ to the next term in a generating sequence, satisfying the conclusions of Theorem 2.2.3.

We will consider the (modified) algorithm of Theorem 2.2.3 in various rings $R$ with given regular parameters $x, y$. We will denote

$$
\begin{gathered}
P_{i}(R)=P_{i} \text { so } P_{0}(R)=x, P_{1}(R)=y, \\
\bar{n}_{i}(R)=\bar{n}_{i}, U_{i}(R)=U_{i}, \alpha_{i}(R)=\alpha_{i}, f_{i}^{R}(z)=f_{i}(z), d_{i}(R)=d_{i}, n_{i}(R)=n_{i}=d_{i} \bar{n}_{i} .
\end{gathered}
$$

These calculations not only depend on $R$, but on the previous terms $P_{0}, P_{1}, \ldots, P_{i-1}$ constructed in the algorithm. 
We will also consider the algorithm of Theorem 2.5.1 in different rings $R$, with given regular parameters $x, y$, and a generating sequence

$$
x=P_{0}, y=P_{1}, P_{2}, \ldots, P_{i}, \ldots
$$

constructed by the (modified) algorithm of Theorem 2.2.3. This algorithm considers the birational extension ring $R_{1}$ of $R$ defined by (3.5).

The positive integers $\bar{n}_{1}$ and $\omega_{0}(1)$ of Theorem 2.2.3 are defined by the conditions that $\bar{n}_{1} v(y)=\omega_{0}(1) v(x)$ and $\operatorname{gcd}\left(\bar{n}_{1}, \omega_{0}(1)\right)=1$. Choose $a, b \in \mathbb{N}$ so that $\bar{n}_{1} b-\omega_{0}(1) a=1$. Define

$$
x_{1}=\frac{x^{b}}{y^{a}}, \quad y_{1}=\frac{y^{\bar{n}_{1}}}{x^{\omega_{0}(1)}} .
$$

Let

$$
\sigma=\left[y_{1}\right] \in V_{v} / m_{v},
$$

which is nonzero. Then (as is shown in Theorem 2.5.1) $R_{1} / m_{R_{1}}=R / m_{R}[\sigma]$. Theorem 2.5.1 shows that

$$
Q_{0}=x_{1}, Q_{1}=\frac{P_{2}}{x_{1}^{\omega_{0}(1) n_{1}}}
$$

are regular parameters in $R_{1}$, and taking

$$
Q_{i}=\frac{P_{i+1}}{Q_{0}^{\omega_{0}(1) n_{1} \cdots n_{i}}}
$$

for $1 \leq i$, the $Q_{i}$ are a generating sequence for $v$ in $R_{1}$ produced by the algorithm of Theorem 2.2.3 (as interpreted by Remark 2.2.4).

We will consider the algorithm of Theorem 2.5.1 in different rings $R$, and will denote

$$
Q_{i}\left(R_{1}\right)=Q_{i}, \hat{\beta}_{i}\left(R_{1}\right)=\hat{\beta}_{i}, V_{i}\left(R_{1}\right)=V_{i}, \hat{\alpha}_{i}\left(R_{1}\right)=\hat{\alpha}_{i}
$$

in the notation of the proof of Theorem 2.5.1. 
We have that the $V_{i}\left(R_{1}\right)$ constructed in the the proof of Theorem 2.5.1 for $R \rightarrow R_{1}$ are actually the $U_{i}\left(R_{1}\right)$ as constructed by the algorithm of Theorem 2.2.3.

Let $L_{0} \cong R / m_{R}$ be a coefficient field of $\hat{R}$, so that $\hat{R}=L_{0}[[x, y]]$.

$$
R_{1}=R\left[x_{1}, y_{1}\right]_{m_{v} \cap R\left[x_{1}, y_{1}\right]} .
$$

$v\left(x_{1}\right)>0$ and $v\left(y_{1}\right)=0$. We have that

$$
R_{1} / m_{R_{1}} \cong L_{0}[\sigma]
$$

where $\sigma$ is the class of $y_{1}$ in $R_{1} / m_{R_{1}}$. Let $L_{1} \cong L_{0}(\sigma)$ be a coefficient field of $\hat{R}_{1}$ containing $L_{0}$ (this is possible since $k$ has characteristic zero, by Hensel's Lemma). Let

$$
y_{1}^{*}=y_{1}-\sigma \in m_{\hat{R}_{1}} .
$$

Thus $x_{1}, y_{1}^{*}$ are regular parameters in $\hat{R}_{1}$ and hence

$$
\hat{R}_{1}=L_{1}\left[\left[x_{1}, y_{1}^{*}\right]\right]
$$

We have an expression

$$
x=x_{1}^{\bar{n}_{1}}\left(y_{1}^{*}+\sigma\right)^{a}, y=x_{1}^{\omega_{0}(1)}\left(y_{1}^{*}+\sigma\right)^{b}
$$

in $\hat{R}_{1}$.

\subsection{Monomial Forms under Sequences of Quadratic Trans- forms}

Suppose that $\mathrm{f}$ is a field of characteristic zero, and $K \rightarrow K^{*}$ is an extension of two dimensional algebraic function fields over $\ldots$. Suppose that $v^{*}$ is a rational 
0-dimensional valuation of $K^{*}$ which restricts to $v$. Suppose that $R$ and $S$ are regular algebraic local rings of $K$ and $K^{*}$ respectively such that $v^{*}$ dominates $S$ and $S$ dominates $R$.

By Theorem 5.1 [13] and Theorem 4.8 [18] (summarized after (3.2)), there exists a sequence of quadratic transforms along $v^{*}$

$$
\begin{array}{lll}
R^{\prime} & \rightarrow & S^{\prime} \\
\uparrow & & \uparrow \\
R & \rightarrow & S
\end{array}
$$

such that $R^{\prime} \rightarrow S^{\prime}$ is strongly monomial. For this type of valuation, this means that $R^{\prime}$ has a regular system of parameters $u, v$ and $S^{\prime}$ has a regular system of parameters $x, y$ giving an expression

$$
u=\gamma_{0} x^{t}, v=y
$$

where $\gamma_{0}$ is a unit in $S^{\prime}$. For the rest of this section, we will assume that $R \rightarrow S$ is strongly monomial (so $R, S$ have regular parameters satisfying (3.15)), but we do not assume that $R \rightarrow S$ is stable.

Lemma 3.5.1 Suppose that $R$ has regular parameters $u, v$ and $S$ has regular parameters $x, y$ giving an expression

$$
u=\gamma_{0} x^{t}, v=y
$$

where $\gamma_{0}$ is a unit in $S$. Let $R \rightarrow R_{1}$ be the sequence of quadratic transforms along $v$ defined by (3.5) and Let $S \rightarrow S_{1}$ be the sequence of quadratic transforms along $v^{*}$ defined by (3.5). Then $R_{1}$ has regular parameters $u_{1}, \tilde{v}_{1}$ and $S_{1}$ has regular parameters $x_{1}, \tilde{y}_{1}$ such that

$$
u_{1}=\gamma_{1} x_{1}^{t_{1}}, \tilde{v}_{1}=\tilde{y}_{1}
$$

where $\gamma_{1}$ is a unit in $S_{1}$. 
Proof We use the notation of the previous section. We have that

$$
\begin{gathered}
R_{1}=R\left[u_{1}, v_{1}\right]_{m_{v} \cap R\left[u_{1}, v_{1}\right]}, \\
u=u_{1}^{\bar{n}_{1}(R)} v_{1}^{a(R)}, v=u_{1}^{\omega_{0}(1)(R)} v_{1}^{b(R)}
\end{gathered}
$$

with

$$
\bar{n}_{1}(R) b(R)-\omega_{0}(1)(R) a(R)=1 .
$$

We have that

$$
v_{1}=\frac{v^{\bar{n}_{1}(R)}}{u^{\omega_{0}(1)(R)}}
$$

so that $v\left(v_{1}\right)=0$ and $\left[v_{1}\right]=\sigma(R)$ in $V_{v} / m_{v}$. We have

$$
u_{1}=\frac{u^{b(R)}}{v^{a(R)}} .
$$

Further,

$$
S_{1}=S\left[x_{1}, y_{1}\right]_{m_{v^{*}} \cap S\left[x_{1}, y_{1}\right]}
$$

where

$$
x=x_{1}^{\bar{n}_{1}(S)} y_{1}^{a(S)}, y=x_{1}^{\omega_{0}(1)(S)} y_{1}^{b(S)}
$$

with $\bar{n}_{1}(S) b(S)-\omega_{0}(1)(S) a(S)=1$. We have that

$$
y_{1}=\frac{y^{\bar{n}_{1}(S)}}{x^{\omega_{0}(1)(S)}}
$$

so that $v^{*}\left(y_{1}\right)=0$, and $\left[y_{1}\right]=\sigma(S)$ in $V_{v^{*}} / m_{v^{*}}$. We have

$$
x_{1}=\frac{x^{b(S)}}{y^{a(S)}} .
$$

Substitute

$$
\begin{aligned}
u_{1} & =u^{b(R)} v^{-a(R)}=\gamma_{0}^{b(R)} x^{t b(R)} y^{-a(R)} \\
& =\gamma_{0}^{b(R)}\left(x_{1}^{\bar{n}_{1}(S)} y_{1}^{a(S)}\right)^{t b(R)}\left(x_{1}^{\omega_{0}(1)(S)} y_{1}^{b(S)}\right)^{-a(R)} \\
& =\gamma_{0}^{b(R)} x_{1}^{\bar{n}_{1}(S) t b(R)-\omega_{0}(1)(S) a(R)} y_{1}^{a(S) t b(R)-a(R) b(S)} .
\end{aligned}
$$

Set $t_{1}=\bar{n}_{1}(S) t b(R)-\omega_{0}(1)(S) a(R)$. Since $v^{*}\left(u_{1}\right)>0, v^{*}\left(x_{1}\right)>0$ and $v^{*}\left(\gamma_{0}\right)=v^{*}\left(y_{1}\right)=$ 0 , we have $t_{1}>0$. 


$$
\begin{gathered}
v_{1}=u^{-\omega_{0}(1)(R)} v^{\bar{n}_{1}(R)}=\left(\gamma_{0} x^{t}\right)^{-\omega_{0}(1)(R)} y^{\bar{n}_{1}(R)} \\
=\gamma_{0}^{-\omega_{0}(1)(R)}\left(x_{1}^{\bar{n}_{1}(S)} y_{1}^{a(S)}\right)^{-t \omega_{0}(1)(R)}\left(x_{1}^{\omega_{0}(1)(S)} y_{1}^{b(S)}\right)^{\bar{n}_{1}(R)} \\
=\gamma_{0}^{-\omega_{0}(1)(R)} x_{1}^{\omega_{0}(1)(S) \bar{n}_{1}(R)-t \omega_{0}(1)(R) \bar{n}_{1}(S)} y_{1}^{b(S) \bar{n}_{1}(R)-a(S) t \omega_{0}(1)(R)} . \\
v^{*}\left(v_{1}\right)=v^{*}\left(y_{1}\right)=v^{*}\left(\gamma_{0}\right)=0 \text { and } v^{*}\left(x_{1}\right)>0 \text { implies } \\
\omega_{0}(1)(S) \bar{n}_{1}(R)-t \omega_{0}(1)(R) \bar{n}_{1}(S)=0 .
\end{gathered}
$$

Since $\bar{n}_{1}(S) b(S)-\omega_{0}(1)(S) a(S) \neq 0$, we have that

$$
\left(\begin{array}{ll}
\bar{n}_{1}(S) & \omega_{0}(1)(S) \\
a(S) & b(S)
\end{array}\right)\left(\begin{array}{l}
-t \omega_{0}(1)(R) \\
\bar{n}_{1}(R)
\end{array}\right) \neq\left(\begin{array}{l}
0 \\
0
\end{array}\right)
$$

Thus

$$
m:=b(S) \bar{n}_{1}(R)-a(S) t \omega_{0}(1)(R) \neq 0 .
$$

We have that $u_{1}, v_{1} \in S_{1}$, so that

$$
R_{1}=R\left[u_{1}, v_{1}\right]_{m_{v} \cap R\left[u_{1}, v_{1}\right]} \subset S_{1}
$$

We have a commutative diagram

$$
\begin{array}{ccc}
\hat{R_{1}}=L_{1}\left[\left[u_{1}, v_{1}^{*}\right]\right] & \rightarrow & \hat{S_{1}}=M_{1}\left[\left[x_{1}, y_{1}^{*}\right]\right] \\
\uparrow & & \uparrow \\
\hat{R}=L[[u, v]] & \rightarrow & \hat{S}=M[[x, y]]
\end{array}
$$

where $L, M, L_{1}, M_{1}$ are coefficient fields of $\hat{R}, \hat{S}, \hat{R_{1}}, \hat{S_{1}}$ such that there are inclusions

$$
\begin{array}{ccc}
L_{1} & \rightarrow & M_{1} \\
\uparrow & & \uparrow \\
L & \rightarrow & M
\end{array}
$$

This is possible (by Hensel's Lemma) since $R, S, R_{1}, S_{1}$ have equicharacteristic zero. 
$y_{1}^{*}=y_{1}-\sigma(S), v_{1}^{*}=v_{1}-\sigma(R)$ are constructed as in (3.14). We compute in $M_{1}\left[\left[x_{1}, y_{1}^{*}\right]\right]$,

$$
y_{1}^{m}=\left(y_{1}^{*}+\sigma(S)\right)^{m}=\sigma(S)^{m}+m \sigma(S)^{m-1} y_{1}^{*}+\frac{m(m-1)}{2 !} \sigma(S)^{m-2}\left(y_{1}^{*}\right)^{2}+\cdots
$$

$\gamma_{0}^{-\omega_{0}(1)(R)}=\beta+x_{1} \Omega$ with $0 \neq \beta \in M_{1}$ and $\Omega \in \hat{S_{1}}$.

In $\hat{S_{1}}$ we have an expression

$$
\begin{aligned}
v_{1} & =\left(\beta+x_{1} \Omega\right)\left(\sigma(S)^{m}+m \sigma(S)^{m-1} y_{1}^{*}+\left(y_{1}^{*}\right)^{2} \Lambda\right) \\
& =\beta \sigma(S)^{m}+\beta m \sigma(S)^{m-1} y_{1}^{*}+x_{1} \Omega^{\prime}+\left(y_{1}^{*}\right)^{2} \Lambda^{\prime}
\end{aligned}
$$

for some $\Lambda \in \hat{S_{1}}, \Omega^{\prime}, \Lambda^{\prime} \in \hat{S_{1}}$. Thus $x_{1}, v_{1}-\beta \sigma(S)^{m}$ are regular parameters in $\hat{S_{1}}$, (and $\left.\beta \sigma(S)^{m}=\sigma(R)\right)$. Hence if $u_{1}, v_{1}^{\prime}$ are regular parameters in $R_{1}$, then $x_{1}, y_{1}^{\prime}=v_{1}^{\prime}$ are regular parameters in $S_{1}$, and we have an expression:

$$
\begin{aligned}
& u_{1}=\gamma_{1} x_{1}^{t_{1}} \\
& v_{1}^{\prime}=y_{1}^{\prime}
\end{aligned}
$$

with $\gamma_{1}$ a unit in $S_{1}$.

By iteration of Lemma 3.5.1 and (3.5), we obtain an infinite sequence

$\begin{array}{lll}\vdots & & \vdots \\ \uparrow & & \uparrow \\ R_{2} & \rightarrow & S_{2} \\ \uparrow & & \uparrow \\ R_{1} & \rightarrow & S_{1} \\ \uparrow & & \uparrow \\ R & & \rightarrow\end{array}$


where each $R_{i}$ has regular parameters $u_{i}, \tilde{v}_{i}$ and each $S_{i}$ has regular parameters $x_{i}, \tilde{y}_{i}$ such that

$$
u_{i}=\gamma_{i} x_{i}^{t_{i}}, \tilde{v}_{i}=\tilde{y}_{i},
$$

where $\gamma_{i}$ is a unit in $S_{i}$.

Let $e=\left|\Gamma_{v^{*}} / \Gamma_{v}\right|$ and $f=\left[V_{v^{*}} / m_{v^{*}}: V_{v} / m_{v}\right]$. If $R \rightarrow S$ is stable, then

$$
t_{i}=e \text { and }\left[S_{i} / m_{S_{i}}: R_{i} / m_{R_{i}}\right]=f
$$

for $i \geq 0$.

\subsection{Construction of a Generating Sequence in $S$ from that of $R$}

In this section, we continue to have the assumptions of Section $\S 3.5$. We further assume that $R \rightarrow S$ is stable. Let

$$
P_{0}(R)=u, P_{1}(R)=v, P_{2}(R), \ldots
$$

be a generating sequence in $R$, constructed by the algorithm of Theorem 2.2.3.

Let $P_{0}(S)=x, P_{1}(S)=y$.

Then we have that the $t$ and $t_{1}$ in Lemma 3.5.1 satisfy

$$
t=\left|\Gamma_{v^{*}} / \Gamma_{v}\right|=t_{1},
$$

and

$$
\left[S / m_{S}: R / m_{R}\right]=\left[V_{v^{*}} / m_{v^{*}}: V_{v} / m_{v}\right]=\left[S_{1} / m_{S_{1}}: R_{1} / m_{R_{1}}\right] .
$$

By the calculations in the previous section, we have that

$$
\left(\begin{array}{ll}
b(R) & -a(R) \\
-\omega_{0}(1)(R) & \bar{n}_{1}(R)
\end{array}\right)\left(\begin{array}{ll}
t & 0 \\
0 & 1
\end{array}\right)\left(\begin{array}{ll}
\bar{n}_{1}(S) & a(S) \\
\omega_{0}(1)(S) & b(S)
\end{array}\right)=\left(\begin{array}{ll}
t_{1} & * \\
0 & m
\end{array}\right) .
$$


Taking determinants and using the fact that $t_{1}=t$ gives $t=t m$ so that $m=1$.

Multiplying (3.22) by

$$
\left(\begin{array}{ll}
\bar{n}_{1}(R) & a(R) \\
\omega_{0}(1)(R) & b(R)
\end{array}\right)
$$

we obtain

$$
\bar{n}_{1}(S)=\bar{n}_{1}(R), \omega_{0}(1)(S)=t \omega_{0}(1)(R)
$$

Since

$$
P_{1}(S)^{\bar{n}_{1}(S)}=P_{1}(R)^{\bar{n}_{1}(R)}
$$

we can take $U_{1}(S)$ to be $U_{1}(R)=u^{\omega_{0}(1)(R)}$, so

$$
U_{1}(S)=u^{\omega_{0}(1)(R)}=\gamma_{0}^{\omega_{0}(1)(R)} x^{t \omega_{0}(1)(R)}=\gamma_{0}^{\omega_{0}(1)(R)} x^{\omega_{0}(1)(S)}
$$

That is, we take $\tau_{1}=\gamma_{0}^{\omega_{0}(1)(R)}$ in (3.6). Thus

$$
\alpha_{1}(S)=\left[\frac{P_{1}(S)^{\bar{n}_{1}(S)}}{U_{1}(S)}\right]=\left[\frac{P_{1}(R)^{\bar{n}_{1}(R)}}{U_{1}(R)}\right]=\left[\frac{v^{\bar{n}_{1}(R)}}{u^{\omega_{0}(1)(R)}}\right]=\alpha_{1}(R),
$$

with the notation of (3.7). We have that $R_{1} / m_{R_{1}}=R / m_{R}[\sigma(R)]$ and $S_{1} / m_{S_{1}}=$ $S / m_{S}[\sigma(S)]$ (with notation of (3.11)).

$$
\alpha_{1}(R)=\left[\frac{v^{\bar{n}_{1}(R)}}{u^{\omega_{0}(1)(R)}}\right]=\sigma(R)
$$

and

$$
\begin{aligned}
\alpha_{1}(S) & =\left[\frac{y^{\bar{n}_{1}(R)}}{u^{\omega_{0}(1)(R)}}\right]=\left[\frac{y^{\bar{n}_{1}(R)}}{\gamma_{0}^{\omega_{0}(1)(R)} x^{t \omega_{0}(1)(R)}}\right] \\
& =\left[\gamma_{0}\right]^{-\omega_{0}(1)(R)}\left[\frac{y^{\bar{n}_{1}(S)}}{x^{\omega_{0}(1)(S)}}\right]=\left[\gamma_{0}\right]^{-\omega_{0}(1)(R)} \sigma(S) .
\end{aligned}
$$

Thus $R_{1} / m_{R_{1}}=R / m_{R}\left(\alpha_{1}(R)\right)$ and $S_{1} / m_{S_{1}}=S / m_{S}\left(\alpha_{1}(R)\right)$.

By (3.21), we have that

$$
\left[S_{1} / m_{S_{1}}: S / m_{S}\right]=\left[R_{1} / m_{R_{1}}: R / m_{R}\right]
$$

and thus

$$
d_{1}(S)=\left[S / m_{S}\left(\alpha_{1}(R)\right): S / m_{S}\right]=\left[R / m_{R}\left(\alpha_{1}(R)\right): R / m_{R}\right]=d_{1}(R),
$$


and the minimal polynomial $f_{1}^{S}(z)$ of $\alpha_{1}(S)$ over $S / m_{S}$ is the minimal polynomial $f_{1}^{R}(z)$ of $\alpha_{1}(R)$ over $R / m_{R}$. Thus

$$
x, y=P_{1}(R), P_{2}(R)
$$

are the first terms of a generating sequence in $S$, obtained by the (modified) algorithm of Theorem 2.2.3.

Proposition 3.6.1 Suppose that $i \geq 2$ and

$$
P_{0}(S)=x, P_{1}(S)=y, P_{2}(S)=P_{2}(R), \ldots, P_{i}(S)=P_{i}(R)
$$

are the first $i+1$ terms of a generating sequence in $S$ produced by the modified algorithm of Theorem 2.2.3. Then

$$
P_{0}(S)=x, P_{1}(S)=y, P_{2}(S)=P_{2}(R), \ldots, P_{i}(S)=P_{i}(R), P_{i+1}(S)=P_{i+1}(R)
$$

are the first $i+2$ terms of a generating sequence in $S$ produced by the modified algorithm of Theorem 2.2.3.

Proof With the assumption, we have that for $j \leq i-1$,

$$
\begin{gathered}
\bar{n}_{j}(S)=\bar{n}_{j}(R), \alpha_{j}(S)=\alpha_{j}(R), \\
d_{j}(S)=\left[S / m_{S}\left(\alpha_{1}(S), \ldots, \alpha_{j}(S)\right): S / m_{S}\left(\alpha_{1}(S), \ldots, \alpha_{j-1}(S)\right)\right] \\
=\left[R / m_{R}\left(\alpha_{1}(R), \ldots, \alpha_{j}(R)\right): R / m_{R}\left(\alpha_{1}(R), \ldots, \alpha_{j-1}(R)\right)\right]=d_{j}(R)
\end{gathered}
$$

and the minimal polynomial $f_{j}^{S}(z)$ of $\alpha_{j}(S)=\alpha_{j}(R)$ over $S / m_{S}\left(\alpha_{1}(S), \ldots, \alpha_{j-1}(S)\right)$ is the minimal polynomial $f_{j}^{R}(z)$ of $\alpha_{j}(R)$ over $R / m_{R}\left(\alpha_{1}(R), \ldots, \alpha_{j-1}(R)\right)$.

Theorem 2.5.1 produces a generating sequence $Q_{0}\left(R_{1}\right)=u_{1}, Q_{1}\left(R_{1}\right), Q_{2}\left(R_{1}\right), \ldots$ in $R_{1}$ from $P_{0}(R), P_{1}(R), P_{2}(R), \ldots$ The generating sequence $Q_{0}\left(R_{1}\right)=$ $u_{1}, Q_{1}\left(R_{1}\right), Q_{2}\left(R_{1}\right), \ldots$ in $R_{1}$ can be produced by the algorithm of Theorem 2.2.3 
from the regular system of parameters $u_{1}, Q_{1}\left(R_{1}\right)$ in $R_{1}$ (as shown in Theorem 2.5.1 and recalled in (3.22) and (3.13)).

Since $R \rightarrow S$ is stable, we have that

$$
u_{1}=\gamma_{1} x_{1}^{t}
$$

for some unit $\gamma_{1} \in S_{1}$, and recalling (3.23), we have that

$$
\omega_{0}(1)(S)=t \omega_{0}(1)(R) .
$$

By the induction hypothesis applied to the stable map $R_{1} \rightarrow S_{1}$, we have that

$$
x_{1}, Q_{1}\left(R_{1}\right), \ldots, Q_{i}\left(R_{1}\right)
$$

are the first $i+1$ terms of a generating sequence in $S_{1}$, produced by the modified algorithm of Theorem 2.2.3 in $S_{1}$.

For $j \geq 1$, let

$$
\bar{n}_{j}\left(R_{1}\right), U_{j}\left(R_{1}\right), \alpha_{j}\left(R_{1}\right), d_{j}\left(R_{1}\right), f_{j}^{R_{1}}(z)
$$

be the calculations of the algorithm of Theorem 2.2.3 in $R_{1}$, obtained in the construction of the generating sequence $Q_{0}\left(R_{1}\right)=u_{1}, Q_{1}\left(R_{1}\right), Q_{2}\left(R_{1}\right), \ldots$.

For $j \leq i$, let

$$
\bar{n}_{j}\left(S_{1}\right), U_{j}\left(S_{1}\right), \alpha_{j}\left(S_{1}\right), d_{j}\left(S_{1}\right), f_{j}^{S_{1}}(z)
$$

be the calculations of the modified algorithm of Theorem 2.2.3 in $S_{1}$, obtained in the construction of the first $i+1$ terms of the generating sequence $x_{1}, Q_{1}\left(R_{1}\right), Q_{2}\left(R_{1}\right), \ldots, Q_{i}\left(R_{1}\right)$ in $S_{1}$. We have that for $j \leq i-1$,

$$
\begin{aligned}
& \bar{n}_{j}\left(S_{1}\right)=\bar{n}_{j}\left(R_{1}\right), U_{j}\left(S_{1}\right)=U_{j}\left(R_{1}\right), \alpha_{j}\left(S_{1}\right)=\alpha_{j}\left(R_{1}\right), \\
& d_{j}\left(S_{1}\right)=d_{j}\left(R_{1}\right), f_{j}^{S_{1}}(u)=f_{j}^{R_{1}}(u) .
\end{aligned}
$$


Since

$$
Q_{0}\left(S_{1}\right)^{\omega_{0}(1)(S)}=Q_{0}\left(R_{1}\right)^{\omega_{0}(1)(R)} \gamma_{1}^{-\omega_{0}(1)(R)}
$$

we have from (3.13) that for $j \leq i-1$,

$$
\begin{aligned}
Q_{j}\left(S_{1}\right) & =\gamma_{1}^{\omega_{0}(1)(R) n_{1}(S) \cdots n_{j}(S)} \frac{P_{j+1}(S)}{Q_{0}\left(R_{1}\right)^{\omega_{0}(1)(R) n_{1}(S) \cdots n_{j}(S)}} \\
& =\gamma_{1}^{\omega_{0}(1)(R) n_{1}(S) \cdots n_{j}(S)} Q_{j}\left(R_{1}\right) .
\end{aligned}
$$

For $j \leq i-1$, we have by (3.13) and (3.26), and then by (3.24) and (3.25), that

$$
\begin{aligned}
v^{*}\left(Q_{j}\left(R_{1}\right)\right) & =v^{*}\left(P_{j+1}(R)\right)-\omega_{0}(1)(R) n_{1}(R) \cdots n_{j}(R) v^{*}\left(u_{1}\right) \\
& =v^{*}\left(P_{j+1}(R)\right)-n_{1}(R) \cdots n_{j}(R) \omega_{0}(1)(S) v^{*}\left(x_{1}\right) .
\end{aligned}
$$

Thus

$$
\begin{aligned}
G\left(v^{*}\left(x_{1}\right), v\left(Q_{1}(R)\right), \ldots, v\left(Q_{j}(R)\right)\right) & =G\left(v^{*}\left(x_{1}\right), v\left(P_{2}(R)\right), \ldots, v\left(P_{j+1}(R)\right)\right) \\
& =G\left(v^{*}(x), v^{*}(y), v\left(P_{2}(R)\right), \ldots, v\left(P_{j+1}(R)\right)\right) \\
& =G\left(v^{*}(x), v\left(P_{1}(R)\right), \ldots, v\left(P_{j+1}(R)\right)\right)
\end{aligned}
$$

since $G\left(v^{*}\left(x_{1}\right)\right)=G\left(v^{*}(x), v^{*}(y)\right)$, as calculated before (2.52) in the proof of Theorem 2.5.1. Thus $\bar{n}_{i-1}\left(S_{1}\right)=\bar{n}_{i}(S)$. We have that $\bar{n}_{i-1}\left(S_{1}\right)=\bar{n}_{i-1}\left(R_{1}\right)$ by (3.26), and $\bar{n}_{i-1}\left(R_{1}\right)=\bar{n}_{i}(R)$ by (2.52) and (2.51) in the proof of Theorem 2.5.1. Thus

$$
\bar{n}_{i}(S)=\bar{n}_{i}(R)
$$

In applying the modified algorithm of Theorem 2.2.3 to extend $x, P_{1}(R), \ldots, P_{i}(R)$ to a generating sequence in $S$, we can thus take $U_{i}(S)=U_{i}(R)$, and then

$$
\alpha_{i}(S)=\left[\frac{P_{i}(S)^{\bar{n}_{i}(S)}}{U_{i}(S)}\right]=\left[\frac{P_{i}(R)^{\bar{n}_{i}(R)}}{U_{i}(R)}\right]=\alpha_{i}(R) .
$$

We have from (3.10) that

$$
y_{1}=\frac{y^{\bar{n}_{1}(S)}}{x^{\omega_{0}(1)(S)}}=\gamma_{0}^{\omega_{0}(1)(R)} \frac{v^{\bar{n}_{1}(R)}}{u^{\omega_{0}(1)(R)}}=\gamma_{0}^{\omega_{0}(1)(R)} v_{1}
$$

Thus

$$
\sigma\left(S_{1}\right)=\left[y_{1}\right]=\left[\gamma_{0}\right]^{\omega_{0}(1)(R)}\left[v_{1}\right]=\left[\gamma_{0}\right]^{\omega_{0}(1)(R)} \alpha_{1}(R)
$$


in $V_{v^{*}} / m_{v^{*}}$, and

$$
S_{1} / m_{S_{1}}=S / m_{S}\left[\alpha_{1}(R)\right] \text { and } R_{1} / m_{R_{1}}=R / m_{R}\left[\alpha_{1}(R)\right] \text {. }
$$

For $1 \leq j \leq i-1$, by (2.57), we have that

$$
\alpha_{j}\left(S_{1}\right)=\alpha_{j}\left(R_{1}\right)=\hat{\alpha}_{j}\left(R_{1}\right)=\alpha_{j+1}(R) \alpha_{1}(R)^{a(R) \omega_{0}(i+1)(R)+b(R) \omega_{1}(i+1)(R)} .
$$

Thus

$$
\begin{aligned}
d_{i-1}\left(S_{1}\right) & =\left[S_{1} / m_{S_{1}}\left(\alpha_{1}\left(S_{1}\right), \ldots, \alpha_{i-1}\left(S_{1}\right)\right): S_{1} / m_{S_{1}}\left(\alpha_{1}\left(S_{1}\right), \ldots, \alpha_{i-2}\left(S_{1}\right)\right)\right] \\
& =\left[S / m_{S}\left(\alpha_{1}(R), \alpha_{2}(R), \ldots, \alpha_{i}(R)\right): S / m_{S}\left(\alpha_{1}(R), \ldots, \alpha_{i-1}(R)\right)\right]=d_{i}(S)
\end{aligned}
$$

and

$$
\begin{aligned}
d_{i-1}\left(R_{1}\right) & =\left[R_{1} / m_{R_{1}}\left(\alpha_{1}\left(R_{1}\right), \ldots, \alpha_{i-1}\left(R_{1}\right)\right): R_{1} / m_{R_{1}}\left(\alpha_{1}\left(R_{1}\right), \ldots, \alpha_{i-2}\left(R_{1}\right)\right)\right] \\
& =\left[R / m_{R}\left(\alpha_{1}(R), \alpha_{2}(R), \ldots, \alpha_{i}(R)\right): R / m_{R}\left(\alpha_{1}(R), \ldots, \alpha_{i-1}(R)\right)\right]=d_{i}(R) .
\end{aligned}
$$

We thus have that $d_{i}(S)=d_{i}(R)$ since $d_{i-1}\left(S_{1}\right)=d_{i-1}\left(R_{1}\right)$ by (3.26). Thus the minimal polynomial $f_{i}^{S}(z)$ of $\alpha_{i}(S)=\alpha_{i}(R)$ over $S / m_{S}\left(\alpha_{1}(R), \ldots, \alpha_{i-1}(R)\right)$ is the minimal polynomial $f_{i}^{R}(z)$ of $\alpha_{i}(R)$ over $R / m_{R}\left(\alpha_{1}(R), \ldots, \alpha_{i-1}(R)\right)$. Thus we can take $P_{i+1}(S)=P_{i+1}(R)$ in the modified algorithm of Theorem 2.2.3.

We obtain the following theorem (Theorem 3.3.1 in Section §3.3). Theorem 3.6.2 is proven by Ghezzi, Ha and Kashcheyeva in [24] when $\mathfrak{f}$ is algebraically closed of characteristic zero.

Theorem 3.6.2 Suppose that $\mathfrak{f}$ is a field of characteristic zero, $v^{*}$ is a rational 0dimensional valuation, $n=2$ and $R \rightarrow S$ is stable. Then

$$
\operatorname{gr}_{v^{*}}(S) \cong\left(\operatorname{gr}_{v}(R) \otimes_{R / m_{R}} S / m_{S}\right)[Z] /\left(Z^{e}-\left[\gamma_{0}\right]^{-1}[u]\right),
$$

and the degree of the extension of quotient fields of $g r_{v}(R) \rightarrow g r_{v^{*}}(S)$ is ef. 
Proof We have an inclusion of graded algebras $\operatorname{gr}_{v}(R) \rightarrow \operatorname{gr}_{v^{*}}(S)$. The classes $\left[P_{i}(R)\right]$ for $i \geq 0$ generate $\operatorname{gr}_{v}(R)$ as a $\operatorname{gr}_{v}(R)_{0}=R / m_{R}$-algebra and the classes $\left[P_{0}(S)\right]$ and $\left[P_{i}(R)\right]$ for $i \geq 1$ generate $\operatorname{gr}_{v^{*}}(S)$ as a $\operatorname{gr}_{v}(S)_{0}=S / m_{S}$-algebra by Theorem 2.2.12 and Proposition 3.6.1. We have the relation

$$
\left[P_{0}(S)\right]^{t}\left[\gamma_{0}\right]=\left[P_{0}(R)\right]
$$

in $\operatorname{gr}_{v^{*}}(S)$. Further,

$$
n_{i}(R)=n_{i}(S) \text { for } i \geq 1
$$

by Proposition 3.6.1.

Since $\operatorname{gr}_{v}(R) \otimes_{R / m_{R}} S / m_{S} \rightarrow \operatorname{gr}_{v^{*}}(S)$ is homogeneous, to verify that it is 1-1, it suffices to show that the homomorphism of $S / m_{S}$-vector spaces

$$
\operatorname{gr}_{\nu}(R)_{\lambda} \otimes_{R / m_{R}} S / m_{S} \rightarrow \mathrm{gr}_{\nu^{*}}(S)_{\lambda}
$$

is 1-1 for all $\lambda \in S^{R}(v)$. By 2) of Theorem 2.2.3, the set of all monomials

$$
\left[P_{0}(R)\right]^{i_{0}}\left[P_{1}(R)\right]^{i_{1}} \cdots\left[P_{r}(R)\right]^{i_{r}}
$$

such that $r \in \mathbb{N}, i_{k} \in \mathbb{N}, 0 \leq i_{k}<n_{k}(R)$ for $1 \leq k \leq r$ and

$$
i_{0} v\left(P_{0}(R)\right)+\cdots+i_{r} v\left(P_{r}(R)\right)=\lambda
$$

is an $R / m_{R}$-basis of $\operatorname{gr}_{v}(R)_{\lambda}$, and the set of all

$$
\left[P_{0}(S)\right]^{j_{0}}\left[P_{1}(S)\right]^{j_{1}} \cdots\left[P_{s}(S)\right]^{j_{s}}
$$

such that $s \in \mathbb{N}, j_{k} \in \mathbb{N}, 0 \leq j_{k}<n_{k}(S)$ for $1 \leq k \leq s$ and

$$
j_{0} v^{*}\left(P_{0}(S)\right)+\cdots+j_{s} v^{*}\left(P_{s}(S)\right)=\lambda
$$

is an $S / m_{S}$-basis of $\operatorname{gr}_{v^{*}}(S)_{\lambda}$. 
By (3.27), (3.28), (3.30) and (3.31), we have that (3.29) is 1-1, so

$$
\operatorname{gr}_{v}(R) \otimes_{R / m_{R}} S / m_{S} \rightarrow \operatorname{gr}_{v^{*}}(S)
$$

is $1-1$.

We have established that $\left[P_{0}(S)\right]$ generates $\operatorname{gr}_{v^{*}}(S)$ as a $\operatorname{gr}_{v}(R) \otimes_{R / m_{R}} S / m_{S^{-}}$ algebra and that the relation (3.27) holds. To establish that the conclusions of the theorem hold, we must show that if there is a relation

$$
h_{0}+\left[P_{0}(S)\right] h_{1}+\cdots+\left[P_{0}(S)\right]^{t-1} h_{t-1}=0
$$

in $\operatorname{gr}_{v^{*}}(S)$, with $h_{i} \in \operatorname{gr}_{v}(R) \otimes_{R / m_{R}} S / m_{S}$, then $h_{0}=h_{1}=\cdots=h_{t-1}=0$. We may assume that each $\left[P_{0}(S)\right]^{j} h_{j}$ is homogeneous of the same degree $\lambda$. Since $R \rightarrow S$ is stable, we have that $t=\left[\Gamma_{v^{*}}: \Gamma_{v}\right]$ and $i v\left(P_{0}(S)\right) \notin \Gamma_{v}$ for $1 \leq i \leq t-1$. Thus there can be at most one nonzero expression in (3.32), so all terms are zero. 


\section{CHAPTER 4}

\section{A SUFFICIENT CONDITION FOR STABLE EXTENSIONS IN DIMENSION TWO}

Throughout this chapter, let $\mathfrak{f}$ be a field of characteristic zero, $K^{*} / K$ be an algebraic extension of 2-dimensional algebraic function fields over $\mathfrak{f}, v$ be a rational 0-dimensional $\mathfrak{f}$-valuation of $K, v^{*}$ be an extension of $v$ to $K^{*}, R$ and $S$ be algebraic local rings of $K$ and $K^{*}$ respectively such that $v^{*}$ dominates $S$ and $S$ dominates $R$ and let the extension $R \rightarrow S$ be strongly monomial; this means that there exist regular parameters $u, v$ in $R$ and $x, y$ in $S$ giving an expression

$$
u=\gamma_{0} x^{t}, v=y \text {, where } \gamma_{0} \text { is a unit in } S \text {. }
$$

In Proposition 3.6.1 in Chapter 3, we show that if $R \rightarrow S$ is stable then the two generating sequences of $R$ and $S$ obtained from the modified algorithm of Theorem 2.2.3 are essentially the same. This, consequently implies that $\operatorname{gr}_{v^{*}}(S)$ is finitely generated as an algebra over $\operatorname{gr}_{v}(R)$ (Theorem 3.6.2). As discussed in Section $\S 3.1$, by Theorem 6.1 [18], the stability of the extension $R \rightarrow S$ can be obtained after a finite number of blowups along $v^{*}$. This is a deep theorem and in general it is very hard to indicate when the blowing up process is actually stable. In dimension two, we will prove that essentially the converse of Proposition 3.6.1 is also true. In Section $\S 4.3$, we give a concrete example of an extension $R \rightarrow S$ that is not stable and for which $S^{S}\left(v^{*}\right)$ is not finitely generated over $S^{R}(v)$. We show that after a few blowups along $v^{*}$, it becomes stable. 


\subsection{The relation between $t_{0}$ and $t_{1}$}

In this section, we adopt the assumptions of Lemma 3.5.1 and the notations from its proof with the only extra convention that $t=t_{0}$. We will investigate more closely the relation between $t_{0}$ and $t_{1}$. Recall from the proof of Lemma 3.5.1 that we have relations

$$
u_{1}=\frac{u^{b\left(R_{0}\right)}}{v^{a\left(R_{0}\right)}}
$$

and

$$
x_{1}=\frac{x^{b\left(S_{0}\right)}}{y^{a\left(S_{0}\right)}} .
$$

From the relations

$$
u=x^{t_{0}} \gamma_{0}, v=y
$$

we get that

$$
\frac{\omega_{0}(1)\left(R_{0}\right)}{\bar{n}_{1}\left(R_{0}\right)}=\frac{v(v)}{v(u)}=\frac{v^{*}(y)}{t_{0} v^{*}(x)}=\frac{\omega_{0}(1)\left(S_{0}\right)}{t_{0} \bar{n}_{1}\left(S_{0}\right)} .
$$

Set $\tilde{t}_{1}=\operatorname{gcd}\left(t_{0}, \omega_{0}(1)\left(S_{0}\right)\right)$, since

$$
\operatorname{gcd}\left(\omega_{0}(1)\left(R_{0}\right), \bar{n}_{1}\left(R_{0}\right)\right)=1=\operatorname{gcd}\left(\omega_{0}(1)\left(S_{0}\right), \bar{n}_{1}\left(S_{0}\right)\right)
$$

we must have that

$$
\tilde{t}_{1} \omega_{0}(1)\left(R_{0}\right)=\omega_{0}(1)\left(S_{0}\right) \text { and } \tilde{t}_{1} \bar{n}_{1}\left(R_{0}\right)=t_{0} \bar{n}_{1}\left(S_{0}\right)
$$

Now recall from the proof of Lemma 2.1 that we have

$$
u_{1}=\left(\text { a unit in } S_{1}\right) \cdot x_{1}^{\bar{n}_{1}\left(S_{0}\right) t_{0} b\left(R_{0}\right)-\omega_{0}(1)\left(S_{0}\right) a\left(R_{0}\right)} .
$$

Using (4.3), we have that

$$
t_{1}=\bar{n}_{1}\left(S_{0}\right) t_{0} b\left(R_{0}\right)-\omega_{0}(1)\left(S_{0}\right) a\left(R_{0}\right)=\tilde{t}_{1}\left(\bar{n}_{1}\left(R_{0}\right) b\left(R_{0}\right)-\omega_{0}(1)\left(R_{0}\right) a\left(R_{0}\right)\right)=\tilde{t}_{1}
$$


So, we have that

$$
\begin{aligned}
& t_{1}=\operatorname{gcd}\left(t_{0}, \omega_{0}(1)\left(S_{0}\right)\right) \\
& \omega_{0}(1)\left(S_{0}\right)=t_{1} \omega_{0}(1)\left(R_{0}\right) \text { and } t_{0} \bar{n}_{1}\left(S_{0}\right)=t_{1} \bar{n}_{1}\left(R_{0}\right) .
\end{aligned}
$$

Remark 4.1.1 From Lemma 3.5.1, we obtain the following:

1. $u_{1}$ and $x_{1}$ are uniquely determined by regular parameters $u, v$ in $R_{1}$ and $x, y$ in $S_{1}$ by the relations

$$
u_{1}=\frac{u^{b(R)}}{v^{a(R)}}, x_{1}=\frac{x^{b(S)}}{y^{a(S)}}
$$

and $u_{1}=\gamma_{1} x_{1}^{t_{1}}$, where $\gamma_{1}$ is a unit in $S_{1}$.

2. For any $w_{1} \in R_{1}$ such that $\left(u_{1}, w_{1}\right)$ is a regular system of parameters of $R_{1},\left(x_{1}, w_{1}\right)$ is also a regular system of parameters of $S_{1}$.

\subsection{The sufficient condition for Stable Extensions of Algebraic Local Rings}

We recall from (3.18) that, by iteration of Lemma 3.5.1 and (3.5), we obtain an infinite sequence

$$
\begin{array}{lll}
\uparrow & & \uparrow \\
R_{2} & \rightarrow & S_{2} \\
\uparrow & & \uparrow \\
R_{1} & \rightarrow & S_{1} \\
\uparrow & & \uparrow \\
R & \rightarrow & S
\end{array}
$$


$R_{1}$ and $S_{1}$ are, respectively, the next local rings in the unique full sequences of quadratic transforms along $v^{*}$ of $R$ and $S$ such that each divisors of $u v$ and of $x y$ in Spec $R_{1}$ and Spec $S_{1}$ has only one component. By Theorem 2.5.1, Lemma 3.5.1 and Remark 4.1.1, for $i \geq 1$, we can inductively specify the regular systems of parameters $\left(u_{i}, \tilde{v}_{i}\right)$ of $R_{i}$ and $\left(x_{i}, \tilde{y}_{i}\right)$ in terms of the regular systems parameters $\left(u_{i-1}, \tilde{v}_{i-1}\right)$ of $R_{i-1}$ and $\left(x_{i-1}, \tilde{y}_{i-1}\right)$ of $S_{i-1}$ as follows. For $i=0, u_{0}=u, \tilde{v}_{0}=v, x_{0}=x$ and $\tilde{y}_{0}=y$ are given. Suppose that the regular systems of parameters $\left(u_{i-1}, \tilde{v}_{i-1}\right)$ of $R_{i-1}$ and $\left(x_{i-1}, \tilde{y}_{i-1}\right)$ are chosen, we construct by the algorithm of Theorem 2.2.3 in $R_{i-1}$ a generating sequence $\left\{P_{j}\left(R_{i-1}\right)\right\}_{j \geq 0}$ of $v$ with

$$
P_{0}\left(R_{i-1}\right)=u_{i-1} \text { and } P_{1}\left(R_{i-1}\right)=\tilde{v}_{i-1}
$$

As in Theorem 2.5.1, we can choose for $R_{i}$ the regular system $\left(u_{i}, \tilde{v}_{i}\right)$ defined by

$$
u_{i}=\frac{u_{i-1}^{b\left(R_{i-1}\right)}}{\tilde{v}_{i-1}^{a\left(R_{i-1}\right)}} \text { and } \tilde{v}_{i}=\frac{P_{2}\left(R_{i-1}\right)}{u_{i}^{\omega_{0}(1)\left(R_{i-1}\right) n_{1}\left(R_{i-1}\right)}}
$$

By Lemma 3.5.1 and Remark 4.1.1, we can choose for $S_{i}$ the regular system $\left(x_{i}, \tilde{y}_{i}\right)$ defined by

$$
x_{i}=\frac{x_{i-1}^{b\left(S_{i-1}\right)}}{\tilde{y}_{i-1}^{a\left(S_{i-1}\right)}} \text { and } \tilde{y}_{i}=\tilde{v}_{i} .
$$

These systems of parameters are related by the relations

$$
u_{i}=\gamma_{i} x_{i}^{t_{i}}, \tilde{v}_{i}=\tilde{y}_{i},
$$

where $\gamma_{i}$ is a unit in $S_{i}$. By convention, $R=R_{0}, S=S_{0}, u=u_{0}, v=\tilde{v}_{0}, x=x_{0}, y=\tilde{y}_{0}$ and $t=t_{0}$. By Theorem 2.5.1, we have that

$$
\begin{aligned}
& P_{j}\left(R_{i}\right)=\frac{P_{j+1}\left(R_{i-1}\right)}{P_{0}\left(R_{i}\right)^{\omega_{0}(1)\left(R_{i-1}\right) n_{1}\left(R_{i-1}\right) \cdots n_{j}\left(R_{i-1}\right)}}, \\
& \bar{n}_{j}\left(R_{i}\right)=\bar{n}_{j+1}\left(R_{i-1}\right) \text { and } d_{j}\left(R_{i}\right)=d_{j+1}\left(R_{i-1}\right) \text { for all } j \geq 1 .
\end{aligned}
$$

For $i \geq 1$, we can write

$$
\frac{v^{*}\left(\tilde{y}_{i}\right)}{v^{*}\left(x_{i}\right)}=\frac{\omega_{0}^{\prime}(1)\left(S_{i}\right)}{\bar{n}_{1}^{\prime}\left(S_{i}\right)}, \text { where } \operatorname{gcd}\left(\omega_{0}^{\prime}(1)\left(S_{i}\right), \bar{n}_{1}^{\prime}\left(S_{i}\right)\right)=1
$$


As analyzed in (4.5) of Section 1 , for all $i \geq 1$, we have that

$$
t_{i+1}=\operatorname{gcd}\left(t_{i}, \omega_{0}^{\prime}(1)\left(S_{i}\right)\right) t_{i+1} \omega_{0}(1)\left(R_{i}\right)=\omega_{0}^{\prime}(1)\left(S_{i}\right) \text { and } t_{i} \bar{n}_{1}^{\prime}\left(S_{i}\right)=t_{i+1} \bar{n}_{1}\left(R_{i}\right) .
$$

In the following lemmas, we assume the following conditions:

$\left(^{*}\right)$ There exist generating sequences $\left\{P_{j}(R)\right\}_{j \geq 0}$ of $v$ in $R$ and $\left\{P_{j}(S)\right\}_{j \geq 0}$ of $v^{*}$ in $S$ obtained by the modified algorithm of Theorem 2.2.3 where

$$
P_{0}(R)=u, P_{1}(R)=v \text { and } P_{0}(S)=x, P_{1}(S)=y
$$

such that

$$
P_{j}(R)=P_{j}(S) \text { for all } j \geq 1 \text {. }
$$

Lemma 4.2.1 Assume condition (*). Then for each $i \geq 0$, there exists a generating sequence $\left\{P_{j}\left(S_{i+1}\right)\right\}_{j \geq 0}$ of $v^{*}$ in $S_{i+1}$ obtained by applying the algorithm of Theorem 2.5.1 for the extension $S_{i} \rightarrow S_{i+1}$ such that:

A(i) $P_{0}\left(S_{i+1}\right)=x_{i+1}$, and for all $j \geq 1$,

$$
\begin{aligned}
& \frac{P_{j+1}\left(S_{i}\right)}{P_{0}\left(S_{i+1}\right)_{0}^{(1)\left(S_{i}\right)\left(S_{i}\right) \cdots n_{j}\left(S_{i}\right)}}=P_{j}\left(S_{i+1}\right)=\left(\text { a unit in } S_{i+1}\right) \cdot P_{j}\left(R_{i+1}\right), \\
& \bar{n}_{j}\left(S_{i+1}\right)=\bar{n}_{j+1}\left(S_{i}\right) \text { and } d_{j}\left(S_{i+1}\right)=d_{j+1}\left(S_{i}\right) ;
\end{aligned}
$$

$B(i) \omega_{0}(1)\left(S_{i+1}\right)=\omega_{0}^{\prime}(1)\left(S_{i+1}\right)$ and $\bar{n}_{1}\left(S_{i+1}\right)=\bar{n}_{1}^{\prime}\left(S_{i+1}\right)$, and

$$
\begin{aligned}
& t_{i+2}=\operatorname{gcd}\left(t_{i+1}, \omega_{0}(1)\left(S_{i+1}\right)\right) \\
& t_{i+2} \omega_{0}(1)\left(R_{i+1}\right)=\omega_{0}(1)\left(S_{i+1}\right) \text { and } t_{i+1} \bar{n}_{1}\left(S_{i+1}\right)=t_{i+2} \bar{n}_{1}\left(R_{i+1}\right) .
\end{aligned}
$$

Proof We proceed by induction on $i$. For $i=0$, Theorem 2.5.1 and equation (4.2) applied to the extension $S_{0} \rightarrow S_{1}$ provides us the following generating sequence 
of $v^{*}$ in $S_{1}$

$$
\begin{aligned}
& P_{0}\left(S_{1}\right)=x_{1}, P_{j}\left(S_{1}\right)=\frac{P_{j+1}\left(S_{0}\right)}{x_{1}^{\omega_{0}(1)\left(S_{0}\right) n_{1}\left(S_{0}\right) \cdots n_{j}\left(S_{0}\right)}}, \\
& \bar{n}_{j}\left(S_{1}\right)=\bar{n}_{j+1}\left(S_{0}\right) \text { and } d_{j}\left(S_{1}\right)=d_{j+1}\left(S_{0}\right) \text { for all } j \geq 1
\end{aligned}
$$

From the hypothesis, we have that $P_{j}\left(R_{0}\right)=P_{j}\left(S_{0}\right)$ for all $j \geq 1$. This implies that $n_{j}\left(S_{0}\right)=n_{j}\left(R_{0}\right)$ for all $j \geq 1$. For all $j \geq 1$, using this relation and (4.5), we have that

$$
\begin{aligned}
& P_{j}\left(S_{1}\right)=\frac{P_{j+1}\left(S_{0}\right)}{x_{1}^{\omega_{0}(1)\left(S_{0}\right) n_{1}\left(S_{0}\right) \cdots n_{j}\left(S_{0}\right)}}=\frac{P_{j+1}\left(R_{0}\right)}{x_{1}^{t_{1} \omega_{0}(1)\left(R_{0}\right) n_{1}\left(R_{0}\right) \cdots n_{j}\left(R_{0}\right)}} \\
& =\gamma_{1}^{\omega_{0}(1)\left(R_{0}\right) n_{1}\left(R_{0}\right) \cdots n_{j}\left(R_{0}\right)} \frac{P_{j+1}\left(R_{0}\right)}{P_{0}\left(R_{1}\right)^{\omega_{0}(1)\left(R_{0}\right) n_{1}\left(R_{0}\right) \cdots n_{j}\left(R_{0}\right)}} \\
& =\left(\text { a unit in } S_{1}\right) \cdot P_{j}\left(R_{1}\right) .
\end{aligned}
$$

Since $P_{0}\left(S_{1}\right)=x_{1}$ and $P_{1}\left(S_{1}\right)=\alpha \cdot P_{1}\left(R_{1}\right)=\alpha \cdot y_{1}$ where $\alpha \in S_{1}$ is a unit, we must have that

$$
\omega_{0}(1)\left(S_{1}\right)=\omega_{0}^{\prime}(1)\left(S_{1}\right) \text { and } \bar{n}_{1}\left(S_{1}\right)=\bar{n}_{1}^{\prime}\left(S_{1}\right)
$$

Combining this with (4.12), we also obtain that

$$
\begin{aligned}
& t_{2}=\operatorname{gcd}\left(t_{1}, \omega_{0}(1)\left(S_{1}\right)\right) \\
& t_{2} \omega_{0}(1)\left(R_{1}\right)=\omega_{0}(1)\left(S_{1}\right) \text { and } t_{1} \bar{n}_{1}\left(S_{1}\right)=t_{2} \bar{n}_{1}\left(R_{1}\right) .
\end{aligned}
$$

Suppose that $A(j)$ and $B(j)$ hold for all $0 \leq j \leq i-1(i \geq 1)$, we will prove $A(i)$ and $B(i)$. We apply the algorithm of Theorem 2.5.1 and equation (4.8) for the extension $S_{i} \rightarrow S_{i+1}$ to get the following generating sequence of $v^{*}$ in $S_{i+1}$

$$
\begin{aligned}
& P_{0}\left(S_{i+1}\right)=x_{i+1}, P_{j}\left(S_{i+1}\right)=\frac{P_{j+1}\left(S_{i}\right)}{x_{i+1}^{\omega_{0}(1)\left(S_{i}\right) n_{1}\left(S_{i}\right) \cdots n_{j}\left(S_{i}\right)}} \\
& \bar{n}_{j}\left(S_{i+1}\right)=\bar{n}_{j+1}\left(S_{i}\right) \text { and } d_{j}\left(S_{i+1}\right)=d_{j+1}\left(S_{i}\right) \text { for all } j \geq 1 .
\end{aligned}
$$

By the inductive hypothesis, we have that $P_{j}\left(S_{i}\right)=$ (a unit in $\left.S_{i}\right) \cdot P_{j}\left(R_{i}\right)$ for all $j \geq 1$. This also implies that $n_{j}\left(R_{i}\right)=n_{j}\left(S_{i}\right)$ for all $j \geq 1$. Moreover, using $B(i-1)$, we have that

$$
t_{i+1} \omega_{0}(1)\left(R_{i}\right)=\omega_{0}(1)\left(S_{i}\right)
$$


For every $j \geq 1$, using a similar calculation as in the case when $i=0$, we obtain

$$
\begin{aligned}
& P_{j}\left(S_{i+1}\right)=\frac{P_{j+1}\left(S_{i}\right)}{w_{i+1}(1)\left(S_{i}\right) n_{1}\left(S_{i}\right) \cdot n_{j}\left(S_{i}\right)}=\frac{\text { (a unit in } \left.S_{i}\right) \cdot P_{j+1}\left(R_{i}\right)}{x_{i+1}^{i+1} \omega_{0}(1)\left(R_{i}\right) n_{1}\left(R_{i}\right) \cdots j_{j}\left(R_{i}\right)} \\
& =\delta_{i+1}^{\left(\omega_{0}(1)\left(R_{i}\right) n_{1}\left(R_{i}\right) \cdots n_{j}\left(R_{i}\right)\right.} \frac{\left(\text { a unit in } S_{i}\right) \cdot P_{j+1}\left(R_{i}\right)}{P_{0}\left(R_{i+1}\right)^{\omega_{0}(1)\left(R_{i}\right) n_{1}\left(R_{i}\right) \cdots n_{j}\left(R_{i}\right)}} \\
& =\left(\text { a unit in } S_{i+1}\right) \cdot P_{j}\left(R_{i+1}\right) \text {. }
\end{aligned}
$$

Thus $A(i)$ is obtained. Finally, since

$$
P_{0}\left(S_{i+1}\right)=x_{i+1} \text { and } P_{1}\left(S_{i+1}\right)=\alpha^{\prime} \cdot P_{1}\left(R_{i+1}\right)=\alpha^{\prime} \cdot y_{i+1}
$$

with $\alpha^{\prime} \in S_{i+1}$ is a unit, we also have that

$$
\omega_{0}(1)\left(S_{i+1}\right)=\omega_{0}^{\prime}(1)\left(S_{i+1}\right) \text { and } \bar{n}_{1}\left(S_{i+1}\right)=\bar{n}_{1}^{\prime}\left(S_{i+1}\right) .
$$

Combining the above relation and (4.12), we get (4.14).

Lemma 4.2.2 Assume condition (*). Then for all $i \geq 1$, we have that $t_{i}=t_{i+1}$.

Proof Fix any $i \geq 1$. From the equations

$$
u_{i+1}=\frac{u_{i}^{b\left(R_{i}\right)}}{v_{i}^{a\left(R_{i}\right)}}, \frac{v\left(v_{i}\right)}{v\left(u_{i}\right)}=\frac{\omega_{0}(1)\left(R_{i}\right)}{\bar{n}_{1}\left(R_{i}\right)} \text { and } \bar{n}_{1}\left(R_{i}\right) b\left(R_{i}\right)-\omega_{0}(1)\left(R_{i}\right) a\left(R_{i}\right)=1 \text {, }
$$

we have that

$$
v\left(u_{i+1}\right)=\frac{1}{\bar{n}_{1}\left(R_{i}\right)} v\left(u_{i}\right) .
$$

Similarly,

$$
v^{*}\left(x_{i+1}\right)=\frac{1}{\bar{n}_{1}^{\prime}\left(S_{i}\right)} v^{*}\left(x_{i}\right)=\frac{1}{\bar{n}_{1}\left(S_{i}\right)} v^{*}\left(x_{i}\right) .
$$

On the other hand, we also have that

$$
v^{*}\left(x_{i+1}\right)=\frac{1}{t_{i+1}} v\left(u_{i+1}\right)=\frac{1}{t_{i+1} \bar{n}_{1}\left(R_{i}\right)} v\left(u_{i}\right)=\frac{t_{i}}{t_{i+1} \bar{n}_{1}\left(R_{i}\right)} v^{*}\left(x_{i}\right) .
$$

Comparing (4.17) and (4.18) we get that

$$
\frac{1}{\bar{n}_{1}\left(S_{i}\right)}=\frac{t_{i}}{t_{i+1} \bar{n}_{1}\left(R_{i}\right)} \text {. }
$$


Now, repeating (4.10) and (4.13), we have that

$$
\begin{aligned}
& \bar{n}_{1}\left(R_{i}\right)=\bar{n}_{2}\left(R_{i-1}\right)=\ldots=\bar{n}_{i+1}\left(R_{0}\right) \\
& \bar{n}_{1}\left(S_{i}\right)=\bar{n}_{2}\left(S_{i-1}\right)=\ldots=\bar{n}_{i+1}\left(S_{0}\right) .
\end{aligned}
$$

Since $P_{j}\left(R_{0}\right)=P_{j}\left(S_{0}\right)$ for all $j \geq 1$, we must have that $\bar{n}_{j}\left(R_{0}\right)=\bar{n}_{j}\left(S_{0}\right)$ for all $j \geq 2$.

Since $i \geq 1$, this implies that $\bar{n}_{i+1}\left(R_{0}\right)=\bar{n}_{i+1}\left(S_{0}\right)$ so that $t_{i}=t_{i+1}$.

Lemma 4.2.3 Assume condition $\left(^{*}\right)$. For all $i \geq 1$, we have that

$$
\left[S_{i} / \mathfrak{m}_{S_{i}}: R_{i} / \mathfrak{m}_{R_{i}}\right]=\left[S_{i+1} / \mathfrak{m}_{S_{i+1}}: R_{i+1} / \mathfrak{m}_{R_{i+1}}\right] .
$$

Proof For each $i \geq 1$, set $f_{i}=\left[S_{i} / \mathrm{m}_{S_{i}}: R_{i} / \mathrm{m}_{R_{i}}\right]$ and consider the diagram

$$
\begin{array}{lll}
S_{i} / \mathrm{m}_{S_{i}} \rightarrow & S_{i+1} / \mathrm{m}_{S_{i+1}} \\
\uparrow & & \uparrow \\
R_{i} / \mathrm{m}_{R_{i}} & \rightarrow & R_{i+1} / \mathrm{m}_{R_{i+1}}
\end{array}
$$

Recall that $\left[R_{i+1} / \mathfrak{m}_{R_{i+1}}: R_{i} / \mathfrak{m}_{R_{i}}\right]=d_{1}\left(R_{i}\right)$ and $\left[S_{i+1} / \mathfrak{m}_{S_{i+1}}: S_{i} / \mathfrak{m}_{S_{i}}\right]=d_{1}\left(S_{i}\right)$. We have that

$$
d_{1}\left(R_{i}\right) f_{i+1}=\left[S_{i+1} / \mathfrak{m}_{S_{i+1}}: R_{i} / \mathfrak{m}_{R_{i}}\right]=d_{1}\left(S_{i}\right) f_{i} .
$$

Iterating (4.10) and (4.13), we also get that

$$
d_{1}\left(R_{i}\right)=d_{i+1}\left(R_{0}\right)=d_{i+1}\left(S_{0}\right)=d_{1}\left(S_{i}\right) \text { for all } i \geq 1 .
$$

From (4.20) and (4.21) we get (4.19).

Theorem 4.2.4 If condition $\left({ }^{*}\right)$ is satisfied then the extension $R_{1} \rightarrow S_{1}$ is stable.

Proof Since $\cup_{i \geq 0} R_{i}=V_{v}$, the stability in Theorem 6.1 [18] must be obtained after some $i_{0} \geq 0$ and this implies that for all $i \geq i_{0}$

$$
t_{i}=e, f_{i}=f, \text { and } \mathbb{Z}^{n} / A_{i}^{t} \mathbb{Z}^{n} \cong \Gamma_{\nu^{*}} / \Gamma_{\nu} .
$$


By Lemma 4.2.2 and Lemma 4.2.3,

$$
t_{i}=t_{i_{0}}=e \text { and } f_{i}=f_{i_{0}}=f \text { for all } i \geq 1 .
$$

Hence, for all $i \geq 1$,

$$
A_{i}=\left(\begin{array}{ll}
t_{i} & 0 \\
0 & 1
\end{array}\right)=\left(\begin{array}{ll}
e & 0 \\
0 & 1
\end{array}\right)
$$

so that $\mathbb{Z}^{n} / A_{i}^{t} \mathbb{Z}^{n} \cong \Gamma_{\nu^{*}} / \Gamma_{\nu}$. In particular $\mathbb{Z}^{n} / A_{1}^{t} \mathbb{Z}^{n} \cong \Gamma_{v^{*}} / \Gamma_{\nu}$.

From the algorithm of Theorem 2.2.3, $\bar{n}_{1}(S)=$ the smallest $m$ such that $m v^{*}(y) \in$ $\left(v^{*}(x)\right)$. On the other hand, we have that $\bar{n}_{1}(R) v^{*}(y)=\bar{n}_{1}(R) v(v) \in(v(u)) \subset(v(x))$. Thus, $\bar{n}_{1}(S) \mid \bar{n}_{1}(R)$. Note that

$$
\alpha_{1}(R)=\frac{v^{\bar{n}_{1}(R)}}{u^{\omega_{0}(1)(R)}}=(\text { a unit of } S) \cdot \frac{y^{\bar{n}_{1}(S)}}{x^{\omega_{0}(1)(S)}}=(\text { a unit of } S) \cdot \alpha_{1}(S)^{\frac{\bar{n}_{1}(R)}{\bar{n}_{1}(S)}}=
$$

Since $P_{j}(R)=P_{j}(S)$ for all $j \geq 1, \alpha_{j}(R)=\alpha_{j}(S)$ for all $j \geq 2$. Thus,

$$
V_{v^{*}} / \mathfrak{m}_{v^{*}}=S / \mathfrak{m}_{S}\left(\alpha_{1}(S), \alpha_{2}(S), \alpha_{3}(S), \ldots\right)=S / \mathfrak{m}_{S}\left(\alpha_{1}(S), \alpha_{2}(R), \alpha_{3}(R), \ldots\right) .
$$

On the other hand,

$$
V_{v} / \mathfrak{m}_{v}=R / \mathfrak{m}_{R}\left(\alpha_{1}(R), \alpha_{2}(R), \alpha_{3}(R), \ldots\right)=R / \mathfrak{m}_{R}\left(\alpha_{1}(S)^{\frac{\bar{n}_{1}(R)}{\bar{n}_{1}(S)}}, \alpha_{2}(R), \alpha_{3}(R), \ldots\right) .
$$

Comparing (4.23) and (4.24), we get that

$$
V_{v^{*}} / \mathfrak{m}_{v^{*}}=V_{v} / \mathfrak{m}_{v}\left(S_{1} / \mathfrak{m}_{S_{1}}\right) .
$$

Lemma 3.1.1, (4.25) and (4.22) imply that $V_{v} / \mathrm{m}_{v}$ and $S_{1} / \mathrm{m}_{S_{1}}$ are linearly disjoint over $R_{1} / \mathrm{m}_{R_{1}}$ in $V_{v^{*}} / \mathrm{m}_{v^{*}}$. We conclude that the extension $R_{1} \rightarrow S_{1}$ is stable.

\subsection{Example}

In section $\S 2.57$ we provide an example of a finite extension $R \rightarrow S$ for which $S^{S}(v)$ is not finitely generated over $S^{R}(v)$. In this section, we give another ex- 
ample of a finite extension $R \rightarrow S$ which is not stable and $S^{S}\left(v^{*}\right)$ is not finitely generated over $S^{S}(v)$. We show by direct calculation that after a few blowups along $v^{*}$, it becomes stable. There is a much shorter proof. However, I am giving another proof since it gives an application of Theorem 4.2.4.

Let $\mathfrak{f}$ be a field of characteristic 0 . Let $R=\mathfrak{f}\left[x^{2}, y\right]_{\left(x^{2}, y\right)}$ whose quotient field is $K=\mathfrak{f}\left(x^{2}, y\right)$ and let $S=\mathfrak{f}[x, y]_{(x, y)}$ whose quotient field is $K^{*}=\mathfrak{f}(x, y)$. We define a rational 0-dimensional valuation $v^{*}$ of $K^{*}$ in terms of its key sequence obtained by the algorithm of Theorem 2.2.3 as described below:

$$
Q_{0}=x, Q_{1}=y, Q_{2}=y^{2}-x^{3}, Q_{3}=Q_{2}^{2}-x^{5} y, Q_{i+1}=Q_{i}^{2}-x^{a_{i}} Q_{i-1} \text { for } i \geq 2,
$$

with the corresponding values

$$
\begin{aligned}
& \gamma_{i}=v^{*}\left(Q_{i}\right), \gamma_{0}=1, \gamma_{1}=1+\frac{1}{2}, \gamma_{2}=3+\frac{1}{2^{2}}, \gamma_{3}=6+\frac{1}{2}+\frac{1}{2^{3}}, \\
& \gamma_{i+1}=2 \gamma_{i}+\frac{1}{2^{i+1}} \text { for } i \geq 1 .
\end{aligned}
$$

In (4.26), the power $a_{i}$ 's are uniquely defined by

$$
a_{i}=v^{*}\left(Q_{i}^{2}\right)-v^{*}\left(Q_{i-1}\right)=2 \gamma_{i}-\gamma_{i-1} \text { for } i \geq 2
$$

Moreover, $\bar{n}_{i}(S)=2, \alpha_{i}(S)=1$ for all $i \geq 1$.

Let $v=\left.v^{*}\right|_{K}$ and let $\left\{P_{i}, \bar{n}_{i}(R), U_{i}(R), \alpha_{i}(R), \beta_{i}\right\}_{i} \geq 0$ be the generating sequence of $v$ obtained by the algorithm of Theorem 2.2.3 with $P_{0}=x^{2}, P_{1}=y$. We have that $v\left(P_{0}\right)=2$ and $v\left(P_{1}\right)=\frac{3}{2}$ so that

$$
\bar{n}_{1}(R)=4, v\left(P_{1}^{4}\right)=v\left(P_{0}^{3}\right) \text { and } \alpha_{1}(R)=\frac{P_{1}^{4}}{P_{0}^{3}}=\frac{y^{4}}{x^{6}}=\alpha_{1}(S)^{2}=1 .
$$

This implies that $P_{2}=P_{1}^{4}-P_{0}^{3}=y^{4}-x^{6}=\left(y^{2}-x^{3}\right)\left(y^{2}+x^{3}\right)$. We see that $P_{2} \neq \delta Q_{2}$ for any unit $\delta \in S$. By Proposition 3.6.1, this implies that the extension $R \rightarrow S$ is not stable. 
Now, we apply the process of Theorem 2.5.1 to get the first quadratic transform $S=S_{0} \rightarrow S_{1}$. In particular, we need to do 3 intermediate quadratic transforms to obtain $S_{1}$ from $S_{0}$ with the corresponding regular systems of parameters and their values as below:

$$
\begin{aligned}
& (x, y) \rightarrow\left(x, \frac{y}{x}\right) \rightarrow\left(\frac{x^{2}}{y}, \frac{y}{x}\right) \rightarrow\left(\frac{x^{2}}{y}, \frac{y^{2}}{x^{3}}-1\right) \\
& \left(1, \frac{3}{2}\right) \rightarrow\left(1, \frac{1}{2}\right) \rightarrow\left(\frac{1}{2}, \frac{1}{2}\right) \rightarrow\left(\frac{1}{2}, \frac{1}{4}\right)
\end{aligned}
$$

We have that $S_{1}=\mathfrak{E}\left[\frac{x^{2}}{y}, \frac{y^{2}}{x^{3}}\right]_{\mathrm{m}_{y^{*}} \cap \mathrm{E}\left[\frac{x^{2}}{y}, \frac{y^{2}}{x^{3}}\right]}$ with regular system of parameters

$$
x_{1}=\frac{x^{2}}{y}, y_{1}=\frac{y^{2}-x^{3}}{x^{3}} .
$$

Similarly, to get the first quadratic transform $R=R_{0} \rightarrow R_{1}$, we need 4 intermediate quadratic transforms with the corresponding regular systems of parameters and their values:

$$
\begin{aligned}
& \left(x^{2}, y\right) \rightarrow\left(\frac{x^{2}}{y}, y\right) \rightarrow\left(\frac{x^{2}}{y}, \frac{y^{2}}{x^{2}}\right) \rightarrow\left(\frac{x^{2}}{y}, \frac{y^{3}}{x^{4}}\right) \rightarrow\left(\frac{x^{2}}{y}, \frac{y^{4}}{x^{6}}-1\right) \\
& \left(2, \frac{3}{2}\right) \rightarrow\left(\frac{1}{2}, \frac{3}{2}\right) \rightarrow\left(\frac{1}{2}, 1\right) \rightarrow\left(\frac{1}{2}, \frac{1}{2}\right) \rightarrow\left(\frac{1}{2}, \frac{1}{4}\right)
\end{aligned}
$$

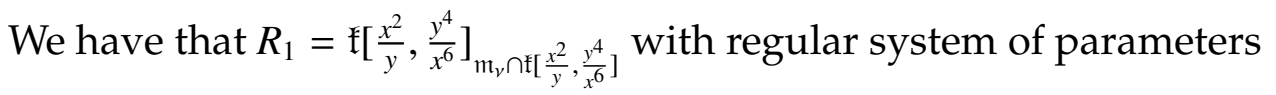

$$
u_{1}=\frac{x^{2}}{y}=x_{1}, v_{1}=\frac{y^{2}+x^{3}}{x^{3}} \cdot y_{1} \text {. }
$$

In (4.29), $\frac{y^{2}+x^{3}}{x^{3}} \in S_{1}$ is a unit so that we can choose $\left(u_{1}, v_{1}\right)$ to be a regular system of parameters of $S_{1}$. This implies that the generating sequence of $v^{*}$ in $S_{1}$ obtained by the algorithm of Theorem 2.2.3 starting with $u_{1}, v_{1}$ coincides with that of $v$ in $R_{1}$ starting with $u_{1}, v_{1}$. By Theorem 4.2.4, the extension $R_{1} \rightarrow S_{1}$ is stable.

As analyzed above, $R_{1}$ and $S_{1}$ share the same generating sequence and this implies that they share the same value semigroup. In the remaining part of this note, we will show that $S^{S}\left(v^{*}\right)$ is not finitely generated over $S^{R}(v)$. Let 
us denote the generating sequences of $v$ in $R_{1}$ and of $v^{*}$ in $S_{1}$ obtained by the algorithm of Theorem 2.2.3 with $P_{0}\left(R_{1}\right)=Q_{0}\left(S_{1}\right)=\frac{x^{2}}{y}, P_{1}\left(R_{1}\right)=\frac{y^{4}}{x^{6}}-1$ and $Q_{1}\left(S_{1}\right)=\frac{y^{2}}{x^{3}}-1$ as $\left\{P_{i}\left(R_{1}\right), \bar{n}_{i}\left(R_{1}\right)\right\}_{i \geq 0}$ and $\left\{Q_{i}\left(S_{1}\right), \bar{n}_{i}\left(S_{1}\right)\right\}_{i \geq 0}$. Let us denote the generating sequences of $v$ in $R_{1}$ and of $v^{*}$ in $S_{1}$ obtained by the algorithm of Theorem 2.5.1 for $R \rightarrow R_{1}$ and $S \rightarrow S_{1}$ starting with regular parameters $\left(x^{2}, y\right)$ in $R$ and $(x, y)$ in $S$ as $\left\{P_{i}^{\prime}\left(R_{1}\right), \bar{n}_{i}^{\prime}\left(R_{1}\right)\right\}_{i \geq 0}$ and $\left\{Q_{i}^{\prime}\left(S_{1}\right), \bar{n}_{i}^{\prime}\left(S_{1}\right)\right\}_{i \geq 0}$. Note that $\left\{P_{i}^{\prime}\left(R_{1}\right), \bar{n}_{i}^{\prime}\left(R_{1}\right)\right\}_{i \geq 0}$ is also the generating sequence of $v$ in $R_{1}$ obtained by the algorithm of Theorem 2.2.3 with

$$
\begin{aligned}
& P_{0}^{\prime}\left(R_{1}\right)=\frac{x^{2}}{y}=P_{0}\left(R_{1}\right), \\
& P_{1}^{\prime}\left(R_{1}\right)=\frac{P_{2}}{P_{0}^{\prime}\left(R_{1}\right)^{3 \cdot 4}}=\left(\frac{y^{2}}{x^{3}}\right)^{6}\left(\frac{y^{4}}{x^{6}}-1\right)=\left(\text { a unit in } R_{1}\right) P_{1}\left(R_{1}\right) .
\end{aligned}
$$

Since $\left\{P_{i}\left(R_{1}\right)\right\}_{i \geq 0}$ and $\left\{P_{i}^{\prime}\left(R_{1}\right)\right\}_{i \geq 0}$ are both obtained from the same algorithm of Theorem 2.2.3 where $P_{0}\left(R_{1}\right)=P_{0}^{\prime}\left(R_{1}\right)$ and $P_{1}\left(R_{1}\right)$ is only different from $P_{1}^{\prime}\left(R_{1}\right)$ a unit in $R_{1}$, we must have that

$$
\bar{n}_{i}\left(R_{1}\right)=\bar{n}_{i}^{\prime}\left(R_{1}\right) \text { for all } i \geq 1 \text {. }
$$

As in the proof of Lemma 4.2.2, we have that

$$
\bar{n}_{i}^{\prime}\left(R_{1}\right)=\bar{n}_{i+1}(R) \text { for all } i \geq 1 .
$$

By (4.30) and (4.31),

$$
\bar{n}_{i}\left(R_{1}\right)=\bar{n}_{i+1}(R) \text { for all } i \geq 1 .
$$

Similarly,

$$
\bar{n}_{i}\left(S_{1}\right)=\bar{n}_{i+1}(S) \text { for all } i \geq 1 \text {. }
$$

As we showed above, $\left\{P_{i}\left(R_{1}\right)\right\}_{i \geq 0}$ is also the generating sequence of $v^{*}$ in $S_{1}$ obtained by the algorithm of Theorem 2.2.3 starting with $P_{0}\left(R_{1}\right), P_{1}\left(R_{1}\right)$ where

$$
P_{0}\left(R_{1}\right)=\frac{x^{2}}{y}=P_{0}\left(S_{1}\right) \text { and } P_{1}\left(R_{1}\right)=\left(\frac{y^{2}}{x^{3}}+1\right) Q_{1}\left(S_{1}\right)=\left(\text { a unit in } S_{1}\right) Q_{1}\left(S_{1}\right) .
$$


This yields that

$$
\bar{n}_{i}\left(R_{1}\right)=\bar{n}_{i}\left(S_{1}\right) \text { for all } i \geq 1 .
$$

From (4.32), (4.33) and (4.34), we get

$$
\bar{n}_{i}(R)=\bar{n}_{i}(S)=2 \text {, for all } i \geq 2 \text {. }
$$

Now, in $R$, we have that $\beta_{0}=2, \beta_{1}=1+\frac{1}{2}, \beta_{2}=6+\frac{1}{2^{2}}$ so that $<\beta_{0}, \beta_{1}, \beta_{2}>\subset \frac{1}{2^{2}} \mathbb{Z}$. $\bar{n}_{3}(R)=2$ yields that $\beta_{3} \in \frac{1}{2^{3}} \mathbb{Z}$ and $<\beta_{0}, \beta_{1}, \beta_{2}, \beta_{3}>\subset \frac{1}{2^{3}} \mathbb{Z}$. By induction, we get

$$
<\beta_{0}, \beta_{1}, \ldots, \beta_{i}>\subset \frac{1}{2^{i}} \mathbb{Z} \text { for all } i \geq 0 .
$$

We have that $\beta_{2}=6+\frac{1}{2}>\left(3+\frac{1}{2}\right)+1=\gamma_{2}+1$. Suppose that $\beta_{i-1}>\gamma_{i-1}+1$ where $i \geq 3$. Since $\alpha_{j}(S)=1$ for all $j \geq 1$, we have no residue field extension:

$$
\mathfrak{f} \subset V_{v} / \mathfrak{m}_{v} \subset V_{v^{*}} / \mathfrak{m}_{v^{*}}=\mathfrak{f}\left(\alpha_{j}(S) \mid j \geq 1\right)=\mathfrak{f} .
$$

Hence,

$$
P_{i}=P_{i-1}^{2}+\text { other terms with lower degrees in } y
$$

so that

$$
\beta_{i}>2 \beta_{i-1}>2\left(\gamma_{i-1}+1\right)>2\left(\gamma_{i-1}+\frac{1}{2^{i}}\right)+1=\gamma_{i}+1 .
$$

By induction, (4.36) holds for all $i \geq 2$.

Finally, for any $i \geq 2$, by (4.35), $<\beta_{0}, \ldots, \beta_{j}>\subset \frac{1}{2^{i-1} \mathbb{Z}}$ for all $j \leq i-1$ so that $\gamma_{i} \notin<\beta_{0}, \ldots, \beta_{j}>$ for all $j \leq i-1$. Moreover, by (4.36), $\beta_{j}>\gamma_{i}$ for all $j \geq i$ so that $\gamma_{i} \notin<\beta_{0}, \ldots, \beta_{j}>$ for all $j \geq i$. So $\gamma_{i} \notin S^{R}(v)$ for all $i \geq 2$. This certainly results that $S^{S}\left(v^{*}\right)$ is not finitely generated over $S^{R}(v)$. 


\section{BIBLIOGRAPHY}

[1] S. Abhyankar, On the Valuations centered in a Local Domain, Amer. J. Math. 78, 1956.

[2] S. Abhyankar, Ramification Theoretic Methods in Algebraic Geometry, Princeton University Press, 1959.

[3] S. Abhyankar, Newton-Puiseux Expansion and generalized Tschirnhausen Transformation I, J. Reine Angew. Math. 260, 1973.

[4] S. Abhyankar, Newton-Puiseux Expansion and generalized Tschirnhausen Transformation II, J. Reine Angew. Math. 261, 1973.

[5] S. Abhyankar, Resolution of Singularities of Embedded Algebraic Surfaces, Academic Press, 1966.

[6] K. Brauner, Klassification der singularitäten algebroider Kurven, Abh. math. semin. Hamburg. Univ 6, 1928.

[7] E. Brieskorn and H. Knörrer, Plane Algebraic Curves, Birkhauäuser, 1986.

[8] A. Campillo, Algebroid Curves in Positive Characteristic, Springer-Verlag, Berlin, Heidelberg, New York, 1980.

[9] V. Cossart and O. Piltant, Resolution of Singularities of Threefolds in Positive Characteristic I. Reduction to Local Uniformization on Artin-Schreier and purely inseparable coverings, J. Algebra 320, No. 3, 2008.

[10] V. Cossart and O. Piltant, Resolution of Singularities of Threefolds in Positive Characteristic II., J. Algebra 321, No. 7, 2009.

[11] S. D. Cutkosky, A skeleton key to Abhyankar's proof of Embedded Resolution of Characteristic p Surfaces, Asian J. Math. 15, No. 3, 2011.

[12] S.D. Cutkosky and O. Kashcheyeva, Algebraic Series and Valuation rings over nonclosed Fields, J. Pure. Appl. Alg. 212, 2008.

[13] S. D. Cutkosky, Local Monomialization and Factorization of Morphisms, Asterisque No. 260, 1999. 
[14] S. D. Cutkoksy, K. Dalili, O. Kashcheyeva, Growth of Rank 1 Valuation Semigroups, Comm. Algebra 38, No. 8, 2010.

[15] S.D. Cutkosky and S. El Hitti, Formal Prime Ideals of infinite value and their Algebraic Resolution, Annales De La Faculté Des Sciences De Toulouse, Mathématiques, 19, 2010.

[16] S.D. Cutkosky and L. Ghezzi, Completions of Valuation Rings, Contemp. math. 386, 2005.

[17] S. D. Cutkosky and O. Kashcheyeva, Algebraic Series and Valuation Rings over nonclosed Fields, J. Pure. Appl. Alg. 212, 2008.

[18] S. D. Cutkosky and O. Piltant, Ramification of Valuations, Adv. Math. 183, 2004.

[19] S.D. Cutkosky and B. Teissier, Semigroups of Valuations on Local Rings, Mich. Math. J. 57, 2008.

[20] S. D. Cutkosky and B. Teissier, Semigroups of Valuations on Local Rings, II, Amer. J. Math. 132, 2010.

[21] S. D. Cutkosky and P. A. Vinh, Ramification of Local Rings along Valuations, preprint, arXiv: 1309.0135, 2013.

[22] S. D. Cutkosky and P. A. Vinh, Valuation Semigroups of Two Dimensional Local Rings, to appear on Proc. London Math. Soc..

[23] S. El Hitti, A Geometric Construction of Minimal Generating Sequences, Master's Thesis, University of Missouri-Columbia, 2006.

[24] L. Ghezzi, T. Ha, O. Kashcheyeva, Toroidalization of Generating Sequences in Dimension Two Function Fields, J. Algebra 301, 2006.

[25] L. Ghezzi and O. Kashcheyeva, Toroidalization of Generating Sequences in Dimension Two Function Fields of Positive Characteristics, J. Pure Appl. Algebra 209, 2007.

[26] R. Goldin and B. Teissier, Resolving Singularities of Plane Analytic Branches with one Toric Morphism, Progr. Math. 181, 2000. 
[27] A. Grothendieck, and A. Dieudonné, Eléments de géométrie algébrique IV, vol. 2, Publ. Math. IHES 24, 1965.

[28] C. Favre and M. Jonsson, The Valuative Tree, Springer, 2004.

[29] W. Heinzer and J. Sally, Extensions of Valuations to the Completion of a Local Domain, Journal of Pure and Applied Algebra 71, 1991.

[30] J. Herzog, Generators and Relations of abelian Semigroups and Semigroup Rings, Manuscript math. 3, 1970.

[31] F. J. Herrera Govantes, M. A. Olalla Acosta, M. Spivakovsky, Valuations in Algebraic Field Extensions, J. Algebra 312, 2007.

[32] H. Knaf and F.-V. Kuhlmann, Abhyankar Places admit Local Uniformization in any Characteristic, Ann. Scient. Ec. Norm. Sup 30, 2005.

[33] F. V. Kuhlmann, Valuation Theoretic Aspects of Local Uniformization, Lecture Notes for the Summer School on Resolution of Singularities, Trieste, Italy, 2006.

[34] F. V. Kuhlmann, Value Groups, Residue Fields, and Bad Places of Rational Function Fields, Trans. Amer. Math. Soc. 356, 2004.

[35] S. Mac Lane, A construction for Absolute Values in Polynomial Rings, Trans. Amer. Math. Soc. 40, 1936.

[36] S. Mac Lane and O. Schilling, Zero-dimensional branches of rank one on Algebraic Varieties, Ann. of Math. 40, 1939.

[37] M. Moghaddam, Realization of a certain class of semi-groups as value semi-groups of valuations, Bull. Iranian Math. Soc. 35, 2009.

[38] M. Nagata, Local Rings, John Wiley and Sons, New York, 1962.

[39] S. Noh, The Value Semigroup of Prime Divisors of the second kind in 2-dim Regular Local Rings, Tran. Amer. Math. Soc 336, 1993.

[40] P. Roquette, History of Valuation Theory, Fields Inst. Commun. 32, 2002.

[41] M. Spivakovsky, Sandwiched Singularities and Desingularization of Surfaces by Normalized Nash Transformations, Ann. of Math. 131, No.3, 1990. 
[42] M. Spivakovsky, Valuations in Function Fields of Surfaces, Amer. J. Math. 112, 1990.

[43] B. Teissier, Overweight Deformations of Affine Toric Varieties and Local Uniformization, preprint.

[44] B. Teissier, Valuations, Deformations and Toric Geometry, dans Valuation Theory and its Applications, Volume II, Fields Inst. Comm. 33, 2003.

[45] M. Vaquié, Extension d'une Valuation, Trans. Amer. Math. Soc. 359, 2007.

[46] M. Vaquié, Valuations and Local Uniformization, Adv. Stud. Pure Math. 43, 2006.

[47] O. Zariski, Algebraic Surfaces, 1935. Second supplemented edition, Ergebnisse der Math. 61, Springer Verlag, 1971.

[48] O. Zariski, Polynomial Ideals defined by Infinitely near Base Points, Amer. J. Math 60, 1938.

[49] O. Zariski, Reduction of the Singularities of Algebraic Three Dimensional Varieties, Ann. of Math. 45, 1944.

[50] O. Zariski, The Reduction of the Singularities of an Algebraic Surface, Ann. of Math. 40, 1939.

[51] O. Zariski and P. Samuel, Commutative Algebra,Volume 2, D.Van Nostrand Company, INC., Princeton, New Jersey, 1960. 


\section{VITA}

Pham An Vinh was born in Quy Nhon, Binh Dinh, Vietnam on July $24^{\text {th }}, 1984$. There, he grew up and received his education from elementary school to college. Influenced by his father, who was a professor of probability at Quy Nhon University, he chose mathematics as his favorite subject since his early childhood. During his high school and college education, he participated and won several prizes in the Vietnamese national annual mathematics olympiards.

In 2006, he graduated from Quy Nhon University with top-ranked GPA in mathematics. His undergraduate thesis was supervised by Professor Ngo Viet Trung, presenting seven different proofs of the Hilbert Nullstellensatz. In 2007, he spent almost one year in Ha Noi, the capital of Vietnam, at the Hanoi Institute of Mathematics in a master program in mathematics. He did not complete his master degree as in Fall 2008, he got admitted to the PhD program in Mathematics of the University of Missouri-Columbia.

At Missouri, he started his study under the supervision of Professor Steven Dale Cutkosky. During his PhD program, he won the first James A. Huckaba Prize in Algebra at the Mathematics Department of the University of MissouriColumbia. His graduation is planned on May 2014. 